\title{
SLOW AND FAST ESCAPE FOR OPEN INTERMITTENT MAPS
}

\author{
MARK F. DEMERS AND MIKE TODD
}

\begin{abstract}
If a system mixes too slowly, putting a hole in it can completely destroy the richness of the dynamics. Here we study this instability for a class of intermittent maps with a family of slowly mixing measures. We show that there are three regimes: 1) standard hyperbolic-like behavior where the rate of mixing is faster than the rate of escape through the hole, there is a unique limiting absolutely continuous conditionally invariant measure (accim) and there is a complete thermodynamic description of the dynamics on the survivor set; 2) an intermediate regime, where the rate of mixing and escape through the hole coincide, limiting accims exist, but much of the thermodynamic picture breaks down; 3) a subexponentially mixing regime where the slow mixing means that mass simply accumulates on the parabolic fixed point. We give a complete picture of the transitions and stability properties (in the size of the hole and as we move through the family) in this class of open systems. In particular we are able to recover a form of stability in the third regime above via the dynamics on the survivor set, even when no limiting accim exists.
\end{abstract}

\section{Introduction and Statement of Results}

Dynamical systems with holes are examples of systems in which the domain is not invariant under the dynamics. Such systems arise in a variety of contexts: For example, in the study of nonattracting invariant sets, as well as in non-equilibrium dynamical systems, in which mass or energy is allowed to enter or escape. In this latter context, a system with a hole can be viewed as a component of a much larger system of interacting components. Examples of such studies include metastable states [KL2, GHW, BV, DoW], coherent sets in nonautonomous systems $\mathrm{FrP}$, and diffusion in extended systems [DGKK].

To date, systems with holes have been studied principally in situations in which the rate of mixing of the closed system (before the introduction of the hole) is exponential and therefore the rate of escape from the system is also exponential. Such systems include expanding maps [PY, CMS, CV, LM], Smale horseshoes [C], Anosov diffeomorphisms [CM], CMT], certain unimodal maps [BDM], and dispersing billiards [DWY1, D3, D4], to name but a few.

In all these papers, the main focus is the existence and physical properties of conditionally invariant measures, which describe the limiting distribution of mass conditioned on non-escape. Given a dynamical system, $(T, X, \mathscr{B})$, one identifies a measurable set $H \in \mathscr{B}$ and studies the open system, $\stackrel{\circ}{T}: \stackrel{\circ}{X} \rightarrow X$, where $\stackrel{\circ}{X}=X \backslash H$. The $n$-step survivor sets are defined by $\stackrel{\circ}{X}^{n}=\bigcap_{i=0}^{n} T^{-i}(\stackrel{\circ}{X})$, which correspond to the non-invariant domains of the iterates of the map, $\stackrel{\circ}{n}^{n}=\left.T^{n}\right|_{X^{n}}$.

Date: November 28, 2016.

MD was partially supported by NSF grant DMS 1362420. This project was started as part of an RiGs grant through ICMS, Scotland. The authors would like to thank ICMS for its generous hospitality. They would also like to thank AIM (workshop on Stochastic Methods for Non-Equilibrium Dynamical Systems) and the ICERM Semester Program on Dimension and Dynamics where some of this work was carried out. We thank the referees for useful suggestions. 
A measure $\mu$ on $X$ is called conditionally invariant if

$$
\frac{\stackrel{\circ}{T}_{*} \mu(A)}{\stackrel{\circ}{*}_{*} \mu(X)}:=\frac{\mu\left(\stackrel{\circ}{X}^{1} \cap T^{-1}(A)\right)}{\mu\left(\stackrel{\circ}{ }^{1}\right)}=\mu(A) \quad \text { for all } A \in \mathscr{B} .
$$

If we set $\mu\left(\stackrel{\circ}{X}^{1}\right)=\lambda$, the relation above can be iterated to obtain $\stackrel{\circ}{*}_{*}^{n} \mu(A)=\lambda^{n} \mu(A)$, so that a conditionally invariant measure necessarily predicts an exponential rate of decay of mass from the open system. Unfortunately, under quite general conditions, uncountably many such measures exist for any eigenvalue $\lambda \in(0,1)$, even if one restricts to measures absolutely continuous with respect to a given reference measure [DY], so existence questions are meaningless.

In order to obtain a physically relevant measure, one fixes a reference measure $m$ and focuses on the existence and properties of limiting distributions obtained by pushing forward $m$ and conditioning on non-escape, i.e. studying limit points of the sequence $\frac{\stackrel{0}{*} n_{n}^{n} m}{m\left(\dot{X}^{n}\right)}$. For systems with exponential rates of escape, such limiting distributions are often conditionally invariant measures which describe the limiting dynamics with respect to a large class of reference measures, and enjoy many of the properties that equilibrium measures enjoy in closed systems. Moreover, in many cases the eigenvalue $\lambda$ associated with such measures describes the exponential rate of escape from the open system with respect to $m$,

$$
-\log \lambda=-\lim _{n \rightarrow \infty} \frac{1}{n} \log m\left(\dot{X}^{n}\right) .
$$

Such limiting distributions have been constructed for all the specific systems listed above, under some assumptions on the size or geometry of the holes.

Recently, there has been interest in open systems exhibiting subexponential rates of escape [DG, APT, DR, FMS, KM], and in particular their relation to slowly mixing systems from non-equilibrium statistical mechanics [Ya. Such open systems exhibit qualitatively different behavior from systems with exponential escape rates. For example, conditionally invariant measures no longer have a physical interpretation as limiting distributions (although arbitrarily many still exist) and limit points of $\frac{i_{*}^{n} m}{m\left(X^{n}\right)}$ are typically singular (with respect to $m$ ) invariant measures supported on $\dot{\circ}^{\infty}:=\bigcap_{i=0}^{\infty} T^{-i}(X \backslash H)$, the set of points which never enter the hole [DF]. From the point of view of limiting distributions, systems with subexponential rates of escape are unstable with respect to leaks in the system.

In the present paper, we introduce holes into a class of Manneville-Pomeau maps $f=f_{\gamma}$ of the unit interval with intermittent behavior. We consider the dynamics of the open system from the point of view of the family of geometric potentials, $t \phi=-t \log |D f|, t \in[0,1]$. When $t=1$, the conformal measure with respect to $\phi=-\log |D f|$ is Lebesgue measure, with respect to which these maps have polynomial rates of mixing. As such, the open system has no physically relevant conditionally invariant measure absolutely continuous with respect to Lebesgue [DF]. But for $t<1$, the maps admit conformal measures $m_{t}$ with respect to $t \phi$ that are exponentially mixing and so have exponential rates of escape, where the mixing rate converges to zero as $t \rightarrow 1$, yielding an excellent test bed for the study of slow mixing with holes.

Fixing a hole $H$ as described in Section 1.2 , we are able to precisely characterize the dynamics of the open system in terms of the parameter $t$ in 3 distinct regimes: $t \in\left[0, t^{H}\right), t \in\left[t^{H}, 1\right)$, and $t=1$, where $t^{H}$ is the Hausdorff dimension of the survivor set.

- When $t \in\left[0, t^{H}\right)$, the escape rate (1.1) with respect to $m_{t}$ is slower than the rate of mixing of the closed system, and so the transfer operator associated with the open system has 
a spectral gap. In this setting, the classical results proved for strongly hyperbolic open systems mentioned above are recovered (Theorem 1.7).

- When $t \in\left[t^{H}, 1\right)$, the escape rate with respect to $m_{t}$ equals the rate of mixing of the closed system and the associated transfer operator for the open system has no spectral gap; however, averaged limit points of the form $\frac{f_{*}^{n} m_{t}}{\left|f_{*}^{n} m_{t}\right|}$, yield conditionally invariant measures, absolutely continuous with respect to $m_{t}$ (Theorem 1.11).

- When $t=1$, the rate of escape with respect to Lebesgue is polynomial and the sequence $\frac{f_{*}^{n} m_{1}}{\left|\dot{f}_{*}^{n} m_{1}\right|}$ converges to the point mass at the neutral fixed point $[\mathrm{DF}]$.

In order to recover a form of stability for the open system when $t=1$, we use an induced map to construct an invariant measure on the survivor set. When $t \in\left[0, t^{H}\right]$, these measures maximize the pressure on the survivor set and satisfy an escape rate formula (see Theorem 1.7). When $t \in\left(t^{H}, 1\right]$, these measures do not maximize the pressure on the survivor set, but they do converge to the absolutely continuous equilibrium state for the closed system as the hole shrinks to a point (Theorem 1.13). Thus, although the system is unstable with respect to leaks from the point of view of the physical limit $\lim _{n \rightarrow \infty} \frac{f_{*}^{n} m_{1}}{\left|\hat{f}_{*}^{n} m_{1}\right|}$ when $t=1$, we are able to recover a type of stability from the point of view of these invariant measures supported on the survivor set.

One of the principal tools we use is a Young tower, which is a type of Markov extension for the open system. It is of independent interest that in order to obtain the sharp division between the regimes listed above, we significantly strengthen previous results on Young towers with holes. Specifically, we prove the existence of a spectral gap for the associated transfer operator under a weak asymptotic condition: The escape rate from the tower is strictly less than the rate of decay in the levels of the tower. Previous results [D1, BDM, DWY1] assumed strong control on the amount of mass lost at each step, while we are able to prove comparable results under this much weaker and more natural condition. Our results are in some sense optimal: When the escape rate equals the rate of decay in the levels of the tower, the essential spectral radius and spectral radius of the associated transfer operator on the relevant function space coincide. This optimality suggests that these results provide a new paradigm for open non-uniformly hyperbolic systems in general.

The paper is organized as follows. In the remainder of Section 1, we state our assumptions precisely, define the relevant terminology and state our main results. In Section 2 we provide some background and initial results on pressure, while in Section 3 we prove an essential inequality relating the escape rate to the difference in pressures between the open and closed systems. In Sections 4, 5 and 6, we prove our main theorems in the three regimes outlined above. Section 7 contains some examples of large holes that do not satisfy our conditions and some analysis of the dynamics in such cases.

1.1. Class of maps. For $\gamma \in(0,1)$, we will study the class of Manneville-Pomeau maps defined by

$$
f=f_{\gamma}: x \mapsto \begin{cases}x\left(1+2^{\gamma} x^{\gamma}\right) & \text { if } x \in[0,1 / 2), \\ 2 x-1 & \text { if } x \in[1 / 2,1] .\end{cases}
$$

Such maps exhibit intermittent behavior due to the neutral fixed point 0 and have been well-studied, most commonly from the point of view of Lebesgue measure [Y2, LSV1], which is the conformal measure with respect to the potential $\phi:=-\log |D f|$. We will be interested in the related family of geometric potentials $t \phi, t \in[0,1]$, and their associated pressures. 
For a dynamical system $(T, X, \mathscr{B})$ with some measurable and metric structure and a measurable potential $\psi: X \rightarrow[-\infty, \infty]$, we define the pressure of this system to be

$$
P(\psi)=P_{T}(\psi):=\sup \left\{h_{T}(\mu)+\int \psi d \mu: \mu \in \mathcal{M}_{T} \text { and }-\int \psi d \mu<\infty\right\} .
$$

Here, $\mathcal{M}_{T}$ is the set of ergodic, $T$-invariant probability measures on $X$ and $h_{\mu}(T)$ is the metric entropy of $\mu$. Note that the restriction on the integral is to deal with cases where the system has infinite entropy, so that the sum defining the pressure may not make sense.

For our class of maps and potentials, we set $p(t):=P(t \phi)$. Note that $p(t)=0$ for $t \geqslant 1$. It is well-known that for $t \leqslant 1$ there exists a unique $(t \phi-p(t))$-conformal measure $m_{t}$ (note that $m_{1}$ is Lebesgue measure). Moreover,

- For $\gamma \in(0,1)$ and $t=1$, there is an equilibrium state $\mu_{1}$ for $\phi$ that is absolutely continuous with respect to $m_{1}$. The system is subexponentially mixing with respect to $\mu_{1}$.

- For $t<1$, there exists a unique equilibrium state $\mu_{t}$ for $t \phi$, which is exponentially mixing and furthermore equivalent to $m_{t}$.

(These facts can be derived from [S3, Proposition 1]; for an alternative perspective on parabolic systems see [MU, Chapter 8].) We will study the dynamics of the related open system with respect to this family of potentials, taking their associated conformal measures $m_{t}$ as our reference measures.

1.2. Introduction of holes. We next introduce a hole $H$ into the system, which in this paper will be a finite union of intervals. The sets $I^{n}=\cap_{i=0}^{n} f^{-i}(I \backslash H), n \geqslant 0$, denote the set of points that have not entered $H$ by time $n$. Define $\stackrel{\circ}{f}=\left.f\right|_{I^{1}}$ to be the map with the hole and its iterates, $f^{n}:=\left.f^{n}\right|_{I^{n}}$. The dynamics of this map define the open system.

A particularly convenient form of hole is defined as follows. Let $\mathcal{P}_{1}$ be the standard renewal partition, i.e., $Z \in \mathcal{P}_{1}$ implies $Z$ is an interval for which either $f(Z) \in \mathcal{P}_{1}$, or $Z=[1 / 2,1)$. We then let $\mathcal{P}_{n}:=\mathcal{P}_{1} \vee\left(\bigvee_{k=0}^{n-1} f^{-k}\{[0,1 / 2),[1 / 2,1)\}\right)$. We fix $N_{0}>0$ and then define a hole to be some collection of elements of $\mathcal{P}_{N_{0}}$ : we call such a set a Markov hole.

Before formulating a condition on the hole, we introduce an induced map $\stackrel{\circ}{F}$, defined as the first return map to $Y=[1 / 2,1) \backslash H$ under $f$. Let $\tau: Y \rightarrow \mathbb{N}$ denote the inducing time, so that either $\stackrel{\circ}{F}(x)=\stackrel{\circ}{f}^{\tau(x)}(x) \in Y$ or $\stackrel{\circ}{F}(x)=\stackrel{\circ}{f}^{\tau(x)}(x) \in H$. In the absence of a hole, $F: Y \rightarrow Y$ would be a full-branched map; however, once a hole has been introduced, $\stackrel{\circ}{F}$ is no longer full branched. Let $\mathcal{Q}$ denote the coarsest partition of $Y$ by images of first returns of elements of $\mathcal{P}_{N_{0}}$. Note that $\mathcal{Q}$ is a finite partition of $Y \backslash H$ due to our definition of $\mathcal{P}_{N_{0}}$.

The classical definitions of transitivity via open sets no longer make sense for the open system 1 (since everything except the hole would be transient), so we adopt the following combinatorial definition in terms of the Markov partition.

We say that $H$ is non-swallowing if:

(1) $\mathcal{Q}$ is transitive on elements: For each pair $Q_{1}, Q_{2} \in \mathcal{Q}, \exists n \in \mathbb{N}$ such that $f^{n}\left(Q_{1}\right) \cap Q_{2} \neq \emptyset$;

(2) For all $\delta>0, m_{1}(\stackrel{\circ}{F}(1 / 2,1 / 2+\delta))>0$.

\footnotetext{
${ }^{1}$ The open system can be decomposed into a disjoint union of open intervals $\stackrel{\circ}{I}=\bigcup_{n=1}^{\infty} \stackrel{\circ}{I}^{n-1} \backslash \stackrel{\circ}{I}^{n}(\bmod 0)$ such that $f\left(\stackrel{\circ}{I}^{n-1} \backslash \stackrel{\circ}{I}^{n}\right)=\stackrel{\circ}{I}^{n-2} \backslash \stackrel{\circ}{I}^{n-1}$ and $f^{n}\left(\stackrel{i}{I}^{n-1} \backslash \stackrel{\circ}{I}^{n}\right) \subset H$.
} 
Otherwise, we call the hole swallowing. Define $X:=Y \backslash H=\cup_{Q \in \mathcal{Q}} Q$. Note that our definition of non-swallowing implies that $\left.\stackrel{\circ}{F}\right|_{X}$ is transitive on elements, but not necessarily aperiodic; however, in Lemma 4.14 we will show that $f$ is aperiodic on elements with this definition.

Condition (2) ensures that the system has repeated passes through a neighborhood of the neutral fixed point: Were this condition violated, much of the non-expansive behavior present in the closed system would be lost or trivialized. Examples of swallowing holes and a brief description of the dynamics in these nontransitive cases are given in Section 7. For our main results, we will assume that our hole is non-swallowing.

Below we record two important facts about the open system that follow from the definition of non-swallowing.

Lemma 1.1. Let $X_{i}, i \in \mathbb{N}$, denote the maximal intervals in $[1 / 2,1]$ on which $\stackrel{\circ}{F}$ is smooth and injective. Then there exists $K_{1}>0$ so that $|\{\tau=n\}| \leqslant K_{1} n^{-\left(1+\frac{1}{\gamma}\right)}$ and $\#\left\{i: \tau\left(X_{i}\right)=n\right\} \leqslant C_{N_{0}}$ for some $C_{N_{0}} \geqslant 1$ depending only on $N_{0}$. Moreover, if $H$ is non-swallowing then there exists $K_{2}>0$ such that for any $N \in \mathbb{N}$, there exists $n \geqslant N$ with $|\{\tau=n\}| \geqslant K_{2} n^{-\left(1+\frac{1}{\gamma}\right)}$

Proof. The first fact follows immediately from standard constructions. See for example, [Y2, LSV1]. The second uses the same constructions, with the added information that there are inducing domains arbitrarily close to $1 / 2$.

Lemma 1.2. If $H$ is a non-swallowing Markov hole then the survivor set $\stackrel{\circ}{I}^{\infty}:=\cap_{n=0}^{\infty} I^{n}$ has positive entropy.

Proof. Let $A$ and $B$ be 1 -cylinders for $\stackrel{\circ}{F}$. By definition of non-swallowing, there exist $n_{A}, n_{B} \in \mathbb{N}$ be such that $f^{n_{A}}(A)=f^{n_{B}}(B)=X$. Then our system contains a horseshoe with entropy at least $\log 2 / \max \left\{n_{A}, n_{B}\right\}>0$.

Given the potential $\phi=-\log |D f|$, set $\phi^{H}$ to be the punctured potential,

$$
\phi^{H}(x)= \begin{cases}\phi(x) & \text { if } x \in I \backslash H, \\ -\infty & \text { if } x \in H .\end{cases}
$$

Then let $p(t)=P(t \phi)$ and $p^{H}(t):=P\left(t \phi^{H}\right)$. Due to the neutral fixed point, it is clear that $p(t) \geqslant p^{H}(t) \geqslant 0$, for $t \in[0,1]$. Also, since $\phi$ is bounded, the condition $-\int \phi^{H} d \nu<\infty$ is equivalent to $\nu(H)=0$, which implies the supremum in $P\left(t \phi^{H}\right)$ is over invariant measures supported on the survivor set, $\stackrel{\circ}{I}^{\infty}$.

1.3. Transfer Operator. We will study the evolution of measures from the point of view of the transfer operators associated with our family of potentials. Given the potential $t \phi$, we define the associated transfer operator acting on $L^{1}\left(m_{t}\right)$ by

$$
\mathcal{L}_{t} f(x):=\mathcal{L}_{t \phi-p(t)} f(x)=\sum_{y \in f^{-1} x} f(y) e^{t \phi(y)-p(t)} .
$$

When we introduce a hole, the transfer operator for the open system corresponds to the transfer operator for the punctured potential,

$$
\stackrel{\circ}{\mathcal{L}}_{t} f(x):=\mathcal{L}_{t \phi^{H}-p(t)} f(x)=\mathcal{L}_{t \phi-p(t)}\left(1_{I^{1}} \cdot f\right)(x)=\sum_{y \in \dot{f}^{-1} x} f(y) e^{t \phi(y)-p(t)} .
$$


Since $m_{t}$ is conformal with respect to $t \phi-p(t)$, we have

$$
\int \dot{\mathcal{L}}_{t}^{n} f d m_{t}=\int_{i^{n}} f d m_{t}
$$

so that the spectral properties of $\dot{\mathcal{L}}_{t}$ are tied to the rate of escape of the open system with respect to $m_{t}$.

1.4. Main results. The standing assumptions of this section are that $f=f_{\gamma}$ is a map as described above and $H$ is a non-swallowing hole.

One of the key quantities associated with an open system is the exponential rate of escape. We will be primarily concerned with the rate of escape with respect to the conformal measures $m_{t}$. To this end we define,

$$
\log \bar{\lambda}_{t}=\limsup _{k} \frac{1}{k} \log m_{t}\left(\stackrel{\circ}{I}^{k}\right) \text { and } \log \underline{\lambda}_{t}=\liminf _{k} \frac{1}{k} \log m_{t}\left(\stackrel{\circ}{I}^{k}\right),
$$

and when these two quantities coincide we denote the common value $\log \lambda_{t}$.

A fundamental relation between pressure and escape is given by the following proposition, which we prove in Section 3 .

Proposition 1.3. For any $t \in[0,1]$, we have

$$
\log \underline{\lambda}_{t} \geqslant p^{H}(t)-p(t) .
$$

In fact, Corollary 1.8 shows that for the class of maps we study here, the inequality above is always an equality.

Whether the punctured pressure $p^{H}(t)$ is positive or zero has a strong influence on the dynamics of the open system with respect to the reference measure $m_{t}$. The following series of results characterizes this behavior in the relevant regimes. To this end, define

$$
t^{H}:=\sup \left\{t \in \mathbb{R}: p^{H}(t)>0\right\} .
$$

By Lemma 1.2, the following result applies in all non-swallowing cases.

Lemma 1.4. If $\stackrel{\circ}{I}^{\infty}$ has positive entropy, then $t^{H} \in(0,1]$ and $p^{H}\left(t^{H}\right)=0$.

Proof. First note that $t \mapsto p^{H}(t)$ is a continuous function of $t$ since $\phi$ is bounded. We have $p^{H}(t) \leqslant p(t)=0$ for all $t \geqslant 1$, and indeed $p^{H}(1)=0$ as well, since for example $h_{\delta_{0}}(f)+\int \phi d \delta_{0}=0$ where $\delta_{0}$ denotes the point mass at 0 . Also, $p^{H}(0)>0$ since the entropy on $\stackrel{\circ}{I}^{\infty}$ is assumed to be positive. Thus, by the continuity of $p^{H}(t), t^{H}$ is finite and is contained in $(0,1]$.

Note that techniques described later in this paper further show that $t^{H}<1$ for any non-trivial hole.

Our next proposition establishes $t^{H}$ as the Hausdorff dimension of the survivor set and describes the behavior of the pressure function on both sides of $t^{H}$. As in [IT2, we say that a potential $\phi$ is recurrent if there exists a finite conservative $(\phi-P(\phi))$-conformal measure; and transient otherwise. Recurrence can also be related to induced potentials. The potential for the induced map $F$ corresponding to $\phi$ is

$$
\Phi:=\sum_{i=0}^{\tau-1} \phi \circ f^{i} .
$$


The following is [T1, Lemma 4.1]. We note that the result only requires that the induced potential $\Phi$ has good distortion properties, for example is locally Hölder (see Section 2.1).

Lemma 1.5. $P(\Phi-P(\phi) \tau) \leqslant 0$.

As described in Section 2.1 below, for our choice of inducing scheme, $\phi$ is recurrent if and only if $P(\Phi-P(\phi) \tau)=0$.

Proposition 1.6. Suppose that $H$ is a non-swallowing Markov hole.

(a) $p^{H}\left(t^{H}\right)=0$ and $P\left(t^{H} \Phi^{H}\right)=0$.

(b) $\operatorname{dim}_{H}\left(I^{\infty}\right)=t^{H}$

(c) $t^{H}>\frac{\gamma}{1+\gamma}$.

(d) If $t>t^{H}$, then $p^{H}(t)=0$ and we have $P\left(t \Phi^{H}-p^{H}(t) \tau\right)<0$. Hence $t \phi^{H}$ is transient.

(e) If $t<t^{H}$, then $p^{H}(t)>0$ and $P\left(t \Phi^{H}-p^{H}(t) \tau\right)=0$. Hence $t \phi^{H}$ is recurrent.

(f) $D p^{H}\left(t^{H}\right)=0$ if $t^{H} \in(\gamma /(1+\gamma), 2 \gamma /(1+\gamma)]$. Otherwis $\oint^{2} D^{-} p^{H}\left(t^{H}\right)<0$.

Proposition 1.6 suggests that $t=t^{H}$ is a dividing line between qualitatively different behaviors of the dynamics with respect to the conformal measures $m_{t}$. The following theorem demonstrates that the dynamics is strongly hyperbolic in the regime $t \in\left[0, t^{H}\right)$. The proof of the theorem uses the induced map $F: Y \circlearrowleft$ to construct an extension of the open system, known as a Young tower, which we denote by $\Delta$. Young towers are defined precisely in Section 4.1 .

Theorem 1.7. (The case $t \in\left[0, t^{H}\right)$ : uniformly hyperbolic behavior.) Fix $q>0$ which will determine the class of $C^{q}$ functions that we will lift to $\Delta$.

If $]^{3}-\log \bar{\lambda}_{t}<p(t)$, then the following hold.

(1) $\lambda_{t}<1$ exists and is the spectral radius of the punctured transfer operator on the tower. The associated eigenvector projects to a nonnegative function $g_{t}$, which is bounded away from zero on $I \backslash H$ and satisfies $\dot{\mathcal{L}}_{t} g_{t}=\lambda_{t} g_{t}$.

(2) $p^{H}(t)=\log \lambda_{t}+p(t)>0$.

(3) There is a unique $\left(\phi^{H}-p^{H}(t)\right)$-conformal measure $m_{t}^{H}$. This is singular with respect to $m_{t}$ and supported on $\stackrel{\circ}{I}^{\infty}$.

(4) The measure $\nu_{t}:=g_{t} m_{t}^{H}$ is an equilibrium state for $t \phi^{H}-p^{H}(t)$. Moreover,

$$
\nu_{t}(\psi)=\lim _{n \rightarrow \infty} \lambda_{t}^{-n} \int_{I^{n}} \psi g_{t} d m_{t}, \quad \text { for all } \psi \in C^{0}(I) .
$$

(5) The measure $\mu_{t}^{H}:=g_{t} m_{t}$ is a conditionally invariant measure with eigenvalue $\lambda_{t}$ and is a limiting distribution in the following sense. Let $\psi \in C^{q}(I)$ satisfy $\psi \geqslant 0$, with $\nu_{t}(\psi)>0$. Then

$$
\left|\frac{\dot{\mathcal{L}}_{t}^{n} \psi}{\left|\dot{\mathcal{L}}_{t}^{n} \psi\right|_{L^{1}\left(m_{t}\right)}}-g_{t}\right|_{L^{1}\left(m_{t}\right)} \leqslant C \sigma^{n}|\psi|_{C^{q}}
$$

for some $C>0$ independent of $\psi$ and $\sigma<1$ depending only on $q$.

\footnotetext{
${ }^{2} \mathrm{By} D^{-}$, we mean the derivative with respect to $t$ from the left.

${ }^{3}$ The condition $-\log \bar{\lambda}_{t}<p(t)$ requires the rate of escape to be slow compared to the pressure, which in this case coincides with the rate of mixing of the closed system with respect to the equilibrium state $\mu_{t}$. For a given hole and fixed values of $\gamma$ and $t$, this condition can be verified numerically: the pressure can be approximated via periodic orbits (for example, by adapting the ideas in [BJP]) and the escape rate can be approximated by volume estimates.
} 
We remark that the convergence to $\mu_{t}^{H}$ described in (5) above holds for a larger class of functions than $C^{q}(I)$. In particular, it holds for $g_{t}^{0}$, where $g_{t}^{0}$ is the invariant density defining the equilibrium state $\mu_{t}$ for the closed system with potential $t \phi-p(t)$; this also implies that the escape rates with respect to both $m_{t}$ and $\mu_{t}$ are the same.

The following variational principle relating the escape rate to the pressure for $t \in[0,1]$ is a simple consequence of Proposition 1.3 and Theorem 1.7. Notice that it also justifies identifying the condition $-\log \bar{\lambda}_{t}<p(t)$ with the condition $t \in\left[0, t^{H}\right)$ in the statement of Theorem 1.7 .

Corollary 1.8. For all $t \in[0,1]$,

$$
-\log \bar{\lambda}_{t}<p(t) \text { if and only if } p^{H}(t)>0 \text { if and only if } t<t^{H} .
$$

Moreover, for all $t \in[0,1], \lambda_{t}$ exists and $\log \lambda_{t}=p^{H}(t)-p(t)$.

Our next result shows that in the regime $t \in\left[0, t^{H}\right)$, the conditionally invariant measures $\mu_{t}^{H}$ we construct vary continuously as the hole shrinks to a point.

Theorem 1.9. Fix $z \in(0,1]$ and let $\left(H_{i}\right)_{i \in \mathbb{N}}$ be a nested sequence of intervals which are nonswallowing Markov holes for $f$ and for which $\cap_{i>0} H_{i}=\{z\}$. Then letting $-\log \lambda_{t}^{H_{i}}$ denote the associated escape rate and $g_{t}^{H_{i}}$ denote the (normalized) eigenvector associated to $\lambda_{t}^{H_{i}}$ from Theorem 1.7, we have $\lambda_{t}^{H_{i}} \rightarrow 1$ and $g_{t}^{H_{i}} \rightarrow g_{t}^{0}$ in $L^{1}\left(m_{t}\right)$ as $i \rightarrow \infty$, where $\mu_{t}=g_{t}^{0} m_{t}$ is the unique equilibrium measure for $t \phi-p(t)$.

It follows from Corollary 1.8 and Theorem 1.9 that $p^{H_{i}}(t) \rightarrow p(t)$ and $t^{H_{i}} \rightarrow 1$ as $i \rightarrow \infty$ (see Lemma 6.9). Thus each fixed $t<1$ eventually satisfies $t<t^{H_{i}}$ for all $H_{i}$ sufficiently small and so Theorem 1.9 implies $\mu_{t}$ is stable with respect to small leaks in the system.

Remark 1.10. A natural question in light of the continuity of $\lambda_{t}^{H}$ proved in Theorem 1.9 is whether $\lambda_{t}^{H}$ is differentiable as well (as a function of $H$ ). While on a global scale the graph of the escape rate function forms a devil's staircase [DW], the derivative of the escape rate may still exist as the hole shrinks to a point, as in [BY, KL2]. Although this result is likely to hold in the present setting, a sequence of holes requires a sequence of increasingly refined Markov partitions. Thus proving such a result would require either adapting the approach of $[\mathrm{FP}]$ to the countable state setting, or constructing a uniform sequence of towers over a single base as in [DT]. Since the present paper is already of considerable length, we do not include this result here.

Finally, we fix $H$ and address the regime $t \geqslant t^{H}$, where we obtain weaker results than Theorem 1.7 due to the absence of a spectral gap.

Theorem 1.11. For each $t \in[0,1)$, all limit points of the sequence $\left\{\frac{\dot{\mathcal{L}}_{t}^{n} 1}{\left|\dot{\mathcal{L}}_{t}^{n} 1\right|_{L^{1}\left(m_{t}\right)}}\right\}_{n \in \mathbb{N}}$ are absolutely continuous with respect to $m_{t}$ with log-Holder continuous densities on elements of $\mathcal{P}_{N_{0}}$.

Moreover, setting $a_{j}=j^{t\left(1+\frac{1}{\gamma}\right)-1} \lambda_{t}^{-j}$ and $Z_{n}=\sum_{j=1}^{n} a_{j}\left|\dot{\mathcal{L}}_{t}^{j} 1\right|_{L^{1}\left(m_{t}\right)}$, all limit points of the averages $\frac{1}{Z_{n}} \sum_{i=1}^{n} a_{i} \mathcal{L}_{t}^{i} 1$ are absolutely continuous conditionally invariant measures with eigenvalue $\lambda_{t}$, and the averages converge in $L^{1}\left(m_{t}\right)$.

Although Theorem 1.11 applies to all $t \in[0,1)$, in light of Theorem 1.7 , it only gives new information for $t \in\left[t^{H}, 1\right)$. It may be of independent interest that the proof of absolute continuity for all limit points of $\left\{\frac{\dot{\mathcal{L}}_{t}^{n} 1}{\left|\mathcal{L}_{t}^{n} 1\right|_{L^{1}\left(m_{t}\right)}}\right\}_{n \in \mathbb{N}}$ holds independently of the proof of Theorem 1.7 . This is in sharp 
contrast to the case $t=1$ for which $\frac{f_{*}^{n} m_{1}}{\left|f_{*}^{n} m_{1}\right|}$ converges weakly to the point mass at the neutral fixed point $[\mathrm{DF}]$.

Next we turn our attention to invariant measures on the survivor set. By Theorem 1.7, we have $\nu_{t}$, an equilibrium measure for $t \phi^{H}-p^{H}(t)$ for $t<t^{H}$ that is supported on $I^{\infty}$. Our next result shows that in fact, one can construct physically meaningful invariant measures on $\stackrel{\circ}{ }^{\infty}$ even for $t \geqslant t^{H}$, including for $t=1$, which are not simply the point mass at the neutral fixed point and indeed contain no atoms. For $t>t^{H}$, these measures do not maximize pressure on the survivor set (in this regime the point mass at 0 does this), but they do converge to the equilibrium measure for the unpunctured potential $t \phi-p(t)$ as the hole shrinks to a point.

In order to obtain sufficient expansion for our map, we will consider the induced map $\stackrel{\circ}{F}$ and work with $\stackrel{\circ}{F}^{2}$ rather than $\stackrel{\circ}{F}$. The induced hole for $F$ is defined by $\tilde{H}=H$ if $H \subset[1 / 2,1]$ and $\tilde{H}=F^{-1}\left(f^{\tau}(H)\right)$ if $H \subset[0,1 / 2]$, where $\tau$ is the first hitting time to $Y$. Since we will be working with $\stackrel{\circ}{F}^{2}$, the hole will effectively be $\tilde{H} \cup F^{-1}(\tilde{H})$, which always has countably many connected components in $Y=[1 / 2,1]$. Let $Y_{n} \subset Y$ be such that $f\left(Y_{n}\right)=J_{n}$, i.e. these are 1-cylinders for $F$ before the introduction of the hole. Set $Y_{i, j}=Y_{i} \cap F^{-1}\left(Y_{j}\right)$ and note that this is the maximal partition on which the return time $\tau^{2}=\tau+\tau \circ F$ is constant.

Fix $z \in(0,1]$ and let $\left(H_{\varepsilon}\right)_{\varepsilon \in\left[0, \varepsilon_{0}\right]}$ be a nested family of intervals (not necessarily elements of a Markov partition) containing $z$ and such that $H_{\varepsilon}$ has length $\varepsilon$. Let $\stackrel{\circ}{F}_{\varepsilon}$ denote the map corresponding to $H_{\varepsilon}$ and let $\dot{Y}_{\varepsilon}^{n}=\cap_{i=0}^{n} F^{-i}\left(Y \backslash \tilde{H}_{\varepsilon}\right)$. Let $\left\{Z_{k, \varepsilon}\right\}_{k \in \mathbb{N}}$ denote the countable collection of maximal intervals on which $\stackrel{\circ}{F}_{\varepsilon}^{2}$ is smooth. Note that each interval $Z_{k, \varepsilon}$ is contained in some $Y_{i, j}$. We shall need the following condition on the family $\left(H_{\varepsilon}\right)_{\varepsilon \leqslant \varepsilon_{0}}$.

(H) Let $\left(H_{\varepsilon}\right)_{\varepsilon \leqslant \varepsilon_{0}}$ be as above. Assume $\inf _{\varepsilon \in\left[0, \varepsilon_{0}\right]} \inf _{k}\left|\stackrel{\circ}{F}_{\varepsilon}^{2}\left(Z_{k, \varepsilon}\right)\right|>0$.

Remark 1.12. Assumption $(\mathbf{H})$ is generically satisfied: if $z$ is not an endpoint of one of the intervals $Y_{n} \subset(1 / 2,1]$ or one of the intervals $J_{n} \subset(0,1 / 2]$, then $(\mathbf{H})$ is satisfied for $\varepsilon_{0}$ sufficiently small.

If one is interested only in the case $t=1$, then one can work with $F$ rather than $F^{2}$ and condition (H) can be stated in terms of $\stackrel{\circ}{F}_{\varepsilon}\left(Y_{n}\right)$. In that case, only the points $z=0$ and $z=1 / 2$ would be excluded by $\mathbf{( H )}$.

For $t \in[0,1]$, recalling the definition of induced potential in 1.2 , let $P_{t, \varepsilon}=P\left(t \Phi-\tau p^{H_{\varepsilon}}(t)\right)$. In Lemma 6.1 we will prove that there exists a $\left(t \Phi-\tau p^{H}(t)-P_{t, \varepsilon}\right)$-conformal measure $\tilde{m}_{t, H}$ for $F$ on $Y$, which has no atoms.

Theorem 1.13. Let $\left(H_{\varepsilon}\right)_{\varepsilon \leqslant \varepsilon_{0}}$ be a nested family of intervals (not necessarily elements of a Markov partition) satisfying $(\mathbf{H})$.

If $\varepsilon_{0}$ is sufficiently small, for $t \in[0,1], \stackrel{\circ}{F}_{\varepsilon}$ admits a physical conditionally invariant measure $\mu_{Y, \varepsilon}$, absolutely continuous with respect to $\tilde{m}_{t, H}$, with eigenvalue $\Lambda_{t, \varepsilon}$. The limit

$$
\nu_{Y, \varepsilon}(\psi)=\lim _{n \rightarrow \infty} \Lambda_{t, \varepsilon}^{-n} \int_{\dot{Y}_{\varepsilon}^{n}} \psi d \mu_{Y, \varepsilon}
$$

exists for each $\psi \in C^{0}(Y)$ and defines an ergodic invariant probability measure $\nu_{Y, \varepsilon}$ supported on the survivor set $\stackrel{\circ}{Y}_{\varepsilon}^{\infty}=\stackrel{\circ}{I}_{\varepsilon}^{\infty} \cap Y$. Moreover,

$$
\log \Lambda_{t, \varepsilon}=P\left(t \Phi^{H_{\varepsilon}}-\tau p^{H_{\varepsilon}}(t)\right)-P\left(t \Phi-\tau p^{H_{\varepsilon}}(t)\right)
$$


and $\nu_{Y, \varepsilon}$ is an equilibrium measure for the potential $t \Phi^{H_{\varepsilon}}-\tau p^{H_{\varepsilon}}(t)-P\left(t \Phi^{H_{\varepsilon}}-\tau p^{H_{\varepsilon}}(t)\right)$.

The measure $\nu_{Y, \varepsilon}$ projects to a probability measure $\nu_{H_{\varepsilon}}$ with the following properties:

(1) $\nu_{H_{\varepsilon}}$ is an invariant measure for $f$ supported on the survivor set $\stackrel{\circ}{I}_{\varepsilon}^{\infty}$;

(2) $\nu_{H_{\varepsilon}}$ is an equilibrium state for $t \phi^{H_{\varepsilon}}-p^{H_{\varepsilon}}(t)-P\left(t \Phi^{H_{\varepsilon}}-\tau p^{H_{\varepsilon}}(t)\right) \cdot 1_{Y}$; so if $t \leqslant t^{H_{\varepsilon}}$ then $\nu_{H_{\varepsilon}}$ is an equilibrium state for $t \phi^{H_{\varepsilon}}-p^{H_{\varepsilon}}(t)$, i.e. it coincides with the measure $\nu_{t}$ from Theorem 1.7;

(3) for fixed $t$, the free energy $h_{\nu_{H_{\varepsilon}}}(f)+\int t \phi^{H_{\varepsilon}} d \nu_{H_{\varepsilon}}$ is continuous in $\varepsilon$ for $\varepsilon \geqslant 0$ close enough to 0 ;

(4) for fixed $\varepsilon$, the free energy of $\nu_{H_{\varepsilon}}=\nu_{H_{\varepsilon}, t}$ is analytic for $t \in\left(t^{H}, 1\right)$ and continuous on the closure, $\left[t^{H_{\varepsilon}}, 1\right]$;

(5) $\nu_{H_{\varepsilon}}$ converges weakly (when integrated against both continuous functions and functions of bounded variation) to the equilibrium measure $\mu_{t}$ for the closed system as $\varepsilon \rightarrow 0$.

Remark 1.14. Note that $\nu_{H_{\varepsilon}}$ is not a measure which maximizes the pressure $h_{\nu}(f)+t \int \phi^{H_{\varepsilon}} d \nu$ on $\stackrel{\circ}{\varepsilon}_{\varepsilon}^{\infty}$ when $t>t^{H_{\varepsilon}}$. One has

$$
0=h_{\delta_{0}}(f)+t \int \phi^{H_{\varepsilon}} d \delta_{0}
$$

where $\delta_{0}$ denotes the point mass at 0 . On the other hand, for the induced system, for any $t \leqslant 1$, we have for the original map $f$,

$$
\begin{aligned}
h_{\nu_{H_{\varepsilon}}}(f)+t \int \phi^{H_{\varepsilon}} d \nu_{H_{\varepsilon}} & =\frac{P\left(t \Phi^{H_{\varepsilon}}-\tau p^{H_{\varepsilon}}(t)\right)}{\int_{Y} \tau d \nu_{Y, \varepsilon}}+p^{H_{\varepsilon}}(t) \\
& =\left(P\left(t \Phi^{H_{\varepsilon}}-\tau p^{H_{\varepsilon}}(t)\right)\right) \nu_{\varepsilon}(Y)+p^{H_{\varepsilon}}(t),
\end{aligned}
$$

where the final equality follows from Kac's formula. Using Proposition 1.6, when $t>t^{H_{\varepsilon}}$ then $p^{H_{\varepsilon}}(t)=0$ and $P\left(t \Phi^{H_{\varepsilon}}\right)<0$ so the right hand side of $(1.4)$ is negative.

Conversely, when $t<t^{H_{\varepsilon}}$ then $p^{H_{\varepsilon}}(t)>0$ and $P\left(t \Phi^{H_{\varepsilon}}-\tau p^{H_{\varepsilon}}(t)\right)=0$, so the right hand side of (1.4) is positive and $\nu_{H_{\varepsilon}}$ is indeed an equilibrium state for $t \phi^{H_{\varepsilon}}-p^{H_{\varepsilon}}(t)$ as stated in Theorem 1.13 .

When $t=t^{H_{\varepsilon}}, p^{H_{\varepsilon}}\left(t^{H_{\varepsilon}}\right)=P\left(t^{H_{\varepsilon}} \Phi^{H_{\varepsilon}}\right)=0$ as in Proposition 1.6 (a), so the right hand side of 1.4 ) is 0 and again $\nu_{H_{\varepsilon}}$ is an equilibrium state for $t \phi^{H_{\varepsilon}}-p^{H_{\varepsilon}}(t)$.

Note that $t=1$ is the only value of $t \in[0,1]$ which is not eventually less than $t^{H}$ as $H$ shrinks to a point. Despite this, the sequence of measures $\nu_{H_{\varepsilon}}$ constructed in Theorem 1.13 converges to the $\mathrm{SRB}$ measure $\mu_{1}$ for the closed system as $\varepsilon \rightarrow 0$. From the point of view of the invariant measure on $\stackrel{\circ}{I}^{\infty}$, then, this theorem recovers a form of stability of the SRB measure for the system in the presence of small leaks. This is in contrast to the instability of the SRB measure from the point of view of limiting distributions in the open system, since $f_{*}^{n} m_{1} /\left|f_{*}^{n} m_{1}\right| \rightarrow \delta_{0}$ as $n \rightarrow \infty[\mathrm{DF}]$.

\section{BASIC PRESSURE RESUlTS}

In this section we will start by recalling thermodynamic formalism for symbolic systems, and then push this onto our system, proving Proposition 1.6.

2.1. Thermodynamic formalism in symbolic spaces. Let $(\Sigma, \sigma)$ be a one-sided Markov shift over the countable alphabet $\mathbb{N}$. This means that there exists a matrix $\left(t_{i j}\right)_{\mathbb{N} \times \mathbb{N}}$ of zeros and ones (with no row and no column made entirely of zeros) such that

$$
\Sigma:=\left\{\left(x_{n}\right)_{n \in \mathbb{N}}: t_{x_{i} x_{i+1}}=1 \text { for every } i \in \mathbb{N}\right\} .
$$


The shift map $\sigma: \Sigma \rightarrow \Sigma$ is defined by $\sigma\left(x_{1} x_{2} x_{2} \ldots\right)=\left(x_{2} x_{2} \ldots\right)$. We will always assume the system $(\Sigma, \sigma)$ to be topologically transitive (but not necessarily mixing), which means that for any two elements $a, b \in \mathbb{N}$, there is a sequence $\left(x_{n}\right)_{n \in \mathbb{N}} \in \Sigma$ with $x_{0}=a$ and $x_{n}=b$ for some $n \in \mathbb{N}$. Note that the theory usually assumes the stronger condition of topological mixing (see [S1 for a precise definition), but in [BS] this was shown to be unnecessary. The space $\Sigma$ endowed with the topology generated by the cylinder sets

$$
C_{i_{1} i_{2} \ldots i_{n}}:=\left\{\left(x_{n}\right)_{n \in \mathbb{N}} \in \Sigma: x_{i}=i_{j} \text { for } j \in\{1,2,3 \ldots n\}\right\},
$$

is a non-compact space. We define the $n^{\text {th }}$ variation of a function $\phi: \Sigma \rightarrow \mathbb{R}$ by

$$
\operatorname{var}_{n}(\phi)=\sup _{\left(i_{1} \ldots i_{n}\right) \in \mathbb{N}^{n}} \sup _{x, y \in C_{i_{1} i_{2} \ldots i_{n}}}|\phi(x)-\phi(y)| .
$$

A function $\phi: \Sigma \rightarrow \mathbb{R}$ is locally Hölder if there exists $0<\gamma<1$ and $C>0$ such that for every $n \in \mathbb{N}$ we have $\operatorname{var}_{n}(\phi) \leq C \gamma^{n}$.

Given a potential $\phi: \Sigma \rightarrow \mathbb{R}$, let $S_{n} \phi(x):=\sum_{k=0}^{n-1} \phi\left(\sigma^{k} x\right)$, be the $n$-th ergodic sum. A measure $\mu$ on $\Sigma$ is called a Gibbs measure for $\phi$ if there exist $K \geqslant 1$ and $P \in \mathbb{R}$ such that, given the $n$-cylinder $C_{i_{1} i_{2} \ldots i_{n}}$, for each $x \in C_{i_{1} i_{2} \ldots i_{n}}$,

$$
\frac{1}{K} \leqslant \frac{\mu\left(C_{i_{1} i_{2} \ldots i_{n}}\right)}{e^{S_{n} \phi(x)-n P}} \leqslant K
$$

Here $P$ is called the Gibbs constant of $\mu$.

The Gurevich Pressure of a locally Hölder potential $\phi: \cup_{n} X_{n} \rightarrow \mathbb{R}$ was introduced by Sarig in [S1], generalizing Gurevich's definition of entropy. It is defined by letting

$$
Z_{n}(\phi)=\sum_{\sigma^{n} x=x} e^{S_{n} \phi(x)} \mathbb{1}_{X_{i}}(x),
$$

where $\mathbb{1}_{X_{i}}(x)$ denotes the characteristic function of the cylinder $X_{i}$, and the Gurevich pressure is

$$
P_{G}(\phi):=\lim _{n \rightarrow \infty} \frac{\log \left(Z_{n}(\phi)\right)}{n},
$$

where the limit exists by almost superadditivity ([S1, Theorem 1]). The limit always exists and its value does not depend on the cylinder $X_{i}$ considered. This notion of pressure satisfies the following variational principle: if $\phi$ is a locally Hölder potential then by [S1, Theorem 3],

$$
P_{G}(\phi)=P(\phi) \text {. }
$$

Hence we can write $P$ in place of $P_{G}$. A measure attaining the supremum above will be called an equilibrium measure for $\phi$.

The potential $\phi$ is called recurrent if

$$
\sum_{n} Z_{n}(\phi) e^{-n P_{G}(\phi)}=\infty
$$

and otherwise it is called transient. Note that due to [S2, Theorem 1], in this setting this definition of transience is equivalent to that given in the previous section. Defining $Z_{n}^{*}(\phi)$ similarly to $Z_{n}(\phi)$, but only summing over those periodic points which make their first return to $X_{i}$ at time $n$, we say that a recurrent potential $\phi$ is positive recurrent if

$$
\sum_{n} n Z_{n}^{*}(\phi) e^{-n P_{G}(\phi)}<\infty
$$

and otherwise $\phi$ is null recurrent. Again this definition is independent of $X_{i}$, and indeed a $k$-cylinder yields the same result. 
It is easy to see from the definition of $P_{G}$ that the pressure function is convex, when finite (one can also easily prove this from the basic definition of pressure $P$ ). Hence we have the following lemma.

Lemma 2.1. Suppose that $\phi: \Sigma \rightarrow \mathbb{R}$ is locally Hölder and $t_{1}<t_{2}$ are such that $P_{G}(t \phi)<\infty$ for $t \in\left(t_{1}, t_{2}\right)$. Then $t \mapsto P_{G}(t \phi)$ is continuous on $\left(t_{1}, t_{2}\right)$.

We say that $(\Sigma, \sigma)$ has the big images and preimages (BIP) property if

$$
\exists b_{1}, \ldots, b_{N} \in \mathbb{N} \text { such that } \forall a \in \mathbb{N}, \exists i, j \text { such that } t_{b_{i} a} t_{a b_{j}}=1 .
$$

A simple example of such a system is the full shift on $\mathbb{N}$. As in [S4], we can set

$$
\tilde{Z}_{n}(\phi)=\sum_{\sigma^{n} x=x} e^{S_{n} \phi(x)}
$$

i.e., we needn't restrict ourselves to $X_{i}$, and it can be shown that

$$
P(\phi)=\lim _{n \rightarrow \infty} \frac{\log \left(\tilde{Z}_{n}(\phi)\right)}{n} .
$$

Moreover, $P(\phi)<\infty$ if and only if $\left.\tilde{Z}_{1}(\phi)\right)<\infty$.

Theorem 2.2 ([S4] $) . P_{G}(\phi)<\infty$ if and only if there is an invariant Gibbs measure $\mu$ for $\phi$ with Gibbs constant $P_{G}(\phi)$. Moreover, if $h(\mu)<\infty$ (equivalently $\int \phi>-\infty$ ) then $\mu$ is an equilibrium state for $\phi$.

Let $C_{n}$ be a cylinder and $\bar{\sigma}: C_{n} \rightarrow C_{n}$ the first return map to $C_{n}$ with return time $r_{C_{n}}$. $\left(C_{n}, \bar{\sigma}\right)$ is known as the induced system on $C_{n}$. Given a potential $\psi: \Sigma \rightarrow \mathbb{R}$, let $\Psi: C_{n} \rightarrow C_{n}$ be defined by $\Psi(x):=S_{r_{C_{n}}} \psi(x)=\sum_{k=0}^{r_{C_{n}}-1} \psi\left(\sigma^{k} x\right)$.

Given a $\sigma$-invariant measure $\mu$, giving positive mass to $C_{n}$, we call $\bar{\mu}=\frac{\left.\mu\right|_{C_{n}}}{\mu\left(C_{n}\right)}$ the lift of $\mu$. By Kac's Lemma, this is $\bar{\sigma}$-invariant. Conversely, given a $\bar{\sigma}$-invariant measure $\bar{\nu}$, if $\nu$ lifts to $\bar{\nu}$, then $\nu$ is called the projection of $\bar{\nu}$. Abramov's formula gives

$$
h(\bar{\nu})=\left(\int r_{C_{n}} d \bar{\nu}\right) h(\nu) \text { and } \int \Psi d \bar{\nu}=\left(\int r_{C_{n}} d \bar{\nu}\right)\left(\int \psi d \nu\right) .
$$

We note that these results also pass to induced maps which are not first return maps.

Given a metric on $\Sigma$, a potential $\psi: \Sigma \rightarrow \mathbb{R}$ is called a metric potential if there exists $K \geqslant 1$ such that

$$
\frac{1}{K} \prod_{j=0}^{n-1} \frac{1}{\psi\left(\sigma^{j} x\right)} \leqslant \operatorname{diam}\left(\left[i_{1}, \ldots, i_{n}\right]\right) \leqslant K \prod_{j=0}^{n-1} \frac{1}{\psi\left(\sigma^{j} x\right)}
$$

The following is [I, Theorem 3.1], adapted slightly. The proof uses inducing to some domain to produce a BIP system, so we change the statement to include this explicitly rather than talking about recurrent points as in [I].

Theorem 2.3. If $(\Sigma, \sigma)$ has the BIP property and is topologically transitive and $\psi$ is a metric potential, then

$$
\operatorname{dim}_{H}(\Sigma)=t^{*}:=\inf \left\{t: P_{G}(-t \log \psi) \leqslant 0\right\}
$$


2.2. Preliminary results on pressure for our systems. For $A$ contained in some interval, we say that the map $T: A \rightarrow A$ is Markov if there exists a countable Markov shift $(\Sigma, \sigma)$ and a continuous bijective map $\pi: \Sigma \rightarrow A$ such that $T \circ \pi=\pi \circ \sigma$. We will use the notation $\left[i_{1}, \ldots, i_{n}\right]:=\pi\left(C_{i_{1} \ldots i_{n}}\right)$. We will also lift potentials $\phi: A \rightarrow \mathbb{R}$ to their symbolic version $\phi \circ \pi$ which we will require to be locally Hölder.

Now we return to our open system $(\stackrel{\circ}{f}, \stackrel{\circ}{I}, H)$. Recall that $X=\cup_{Q \in \mathcal{Q}} Q$ and let $\dot{X}^{\infty}$ denote the set of points which map infinitely often into $X$ under $\stackrel{\circ}{F}$. In the following, we will use the fact that the natural symbolic coding of the system $\stackrel{\circ}{F}: \stackrel{\circ}{X}^{\infty} \rightarrow \stackrel{\circ}{X}^{\infty}$ satisfies the BIP property and is transitive, although it may be not mixing. This means that all the results in Section 2.1 pass to our system: here our potential $\Phi$ lifts to a metric potential on the symbolic model (that is, compatible with the Euclidean metric on $[0,1)$ ), thus also inducing a compatible metric. We also note that any ergodic measure on $I^{\infty}$ with positive entropy must give positive measure to $X$, which, since $\stackrel{\circ}{F}$ is a first return map, by Kac's Lemma means that it must lift to the induced system $(X, \stackrel{\circ}{F})$.

The next lemma follows immediately from the structure of our system.

Lemma 2.4. Suppose that $H$ is a non-swallowing Markov hole. Then

$$
\stackrel{\circ}{I}^{\infty} \backslash\left(\cup_{k \geqslant 0} f^{-k}\left(\dot{X}^{\infty}\right)\right)
$$

consists of at most the countable set of preimages of 0.

We close this section with the proof of our first main result, Proposition 1.6 .

Proof of Proposition 1.6. The fact that $p^{H}\left(t^{H}\right)=0$ is part of Lemma 1.4. The following claim then completes the proof of (a).

Claim. $P\left(t^{H} \Phi^{H}\right)=0$.

Proof. The fact that $P\left(t^{H} \Phi^{H}\right) \leqslant 0$ is Lemma 1.5. Moreover, by definition of $t^{H}$, if $t<t^{H}$ there must be a measure $\mu$ on $I^{\infty}$ of positive entropy with $h(\mu)+\int t \phi d \mu>0$. Since any measure on $I^{\infty}$ of positive entropy lifts to our inducing scheme, Abramov's formula implies that $P\left(t \Phi^{H}\right)>0$. So by the continuity of $t \mapsto P\left(t \Phi^{H}\right)$, when finite (Lemma 2.1), to complete the proof of the claim, we need to show that there is $t<t^{H}$ such that $P\left(t \Phi^{H}\right)<\infty$.

As described in Section 2.1, $P\left(t^{H} \Phi^{H}\right) \leqslant 0$ implies that $\tilde{Z}_{1}\left(t^{H} \Phi\right)<\infty$, which means that $\left.\sum_{i}\left|X_{i}\right|\right|^{H}<$ $\infty$. Since by Lemma 1.1, the diameter of each of the domains in this sum is polynomially small in the inducing time, and the number of domains with the same inducing time is uniformly bounded, this also implies that there exists $t<t^{H}$ such that $\tilde{Z}_{1}(t \Phi)<\infty$. Hence $P\left(t \Phi^{H}\right)<\infty$, as required.

By Theorem 2.3, $\operatorname{dim}_{H}\left(\stackrel{\circ}{X}^{\infty}\right)=t^{H}$. By Lemma 2.4, $\operatorname{dim}_{H}\left(\stackrel{\circ}{I}^{\infty}\right)=\operatorname{dim}_{H}\left(\stackrel{\circ}{X}^{\infty}\right)=t^{H}$, proving (b).

For (c), by Lemma 1.1, we have $\sum_{i}\left|X_{i}\right|^{t}<\infty$ if and only if $t>\frac{\gamma}{1+\gamma}$, since the number of domains with the same inducing time is uniformly bounded by $C_{N_{0}}$. As in the claim, $\sum_{i}\left|X_{i}\right|^{t^{H}}<\infty$ so (c) follows immediately.

We next prove $(\mathrm{d})$. Let $\delta_{0}$ denote the Dirac mass at 0 . Since $H$ is a non-swallowing hole and $h\left(\delta_{0}\right)=\int \phi^{H} d \delta_{0}=0$, the variational definition of pressure implies $p^{H}(t) \geqslant 0$ for all $t \in \mathbb{R}$. The claim implies $p^{H}\left(t^{H}\right)=0$, so since $p^{H}$ is decreasing, $p^{H}(t)=0$ for all $t>t^{H}$. Hence $P\left(t \Phi^{H}-\tau p^{H}(t)\right)=P\left(t \Phi^{H}\right)$ for $t>t^{H}$, so the final part of $(\mathrm{d})$ follows since $t \mapsto P\left(t \Phi^{H}\right)$ is strictly decreasing. 
For (e), by definition $p^{H}(t)>0$. Again as in Lemma $1.5, P\left(t \Phi^{H}-p^{H}(t) \tau\right) \leqslant 0$. By the continuity of pressure, in domains where it is finite (Lemma 2.1) we only need show that for any small $\delta>0$, $0<P\left(t \Phi^{H}-\left(p^{H}(t)-\delta\right) \tau\right)<\infty$. However, from the variational definition of pressure, there must exist an ergodic invariant measure with positive entropy $\mu$ such that

$$
h(\mu)+\int t \phi^{H} d \mu>p^{H}(t)-\delta .
$$

This measure must lift to a measure $\mu_{F}$ on $(Y, F)$. By the Abramov formula,

$$
h\left(\mu_{F}\right)+\int t \Phi^{H}-\left(p^{H}(t)-\delta\right) \tau d \mu_{F}=\left(\int \tau d \mu_{F}\right)\left(h(\mu)+\int t \phi^{H}-\left(p^{H}(t)-\delta\right) d \mu\right)>0 .
$$

Hence the variational principle implies $P\left(t \Phi^{H}-\left(p^{H}(t)-\delta\right) \tau\right)>0$. The fact that this pressure is also finite when $\delta>0$ is small follows from the fact that if $\delta<p^{H}(t)$, then clearly $\tilde{Z}_{1}\left(t \Phi^{H}-\right.$ $\left.\left(p^{H}(t)-\delta\right) \tau\right)<\infty$ since $|\{\tau=n\}|$ is subexponential and the number of domains with $\tau=n$ is uniformly bounded by Lemma 1.1 .

(f) is a standard consequence of the null-recurrence of $t^{H} \phi$, see for example [IT1, Section 8-9].

\section{Proof of Proposition 1.3}

The proof relies on a volume lemma argument (c.f. [Y1, DWY1]) applied to the conformal measures $m_{t}$. However, in order to obtain the volume estimates we need, we shall rely on the following cylinder structure, which is coarser than $\mathcal{P}_{n}$.

Let $\mathcal{D}:=\{[0,1 / 2),[1 / 2,1)\}$ and let $\mathcal{D}_{n}:=\bigvee_{k=0}^{n-1} f^{-k} \mathcal{D}$. Now for $x \in[0,1)$, let $D_{n}(x)$ denote the element of $\mathcal{D}_{n}$ containing $x$. The next lemma follows from 'tempered distortion', see [JR] for a proof.

Lemma 3.1. There exists a sequence $\left(V_{n}\right)_{n} \subset(0, \infty)$ where $V_{n} \rightarrow 0$ as $n \rightarrow \infty$ such that for any $D \in \mathcal{D}_{n}$ and any $x, y \in D,\left|\log D f^{n}(x)-\log D f^{n}(y)\right| \leqslant n V_{n}$.

Using this lemma in conjunction with the Mean Value Theorem, we will estimate the $m_{t}$-measure of elements of $\mathcal{D}_{n}$.

Recalling that $H$ is a union of elements of $\mathcal{P}_{N_{0}}$, let $\hat{N}_{0}$ be such that $H$ is a union of elements of $\mathcal{D}_{\hat{N}_{0}}$.

Suppose $\nu$ is an ergodic invariant probability measure for $\stackrel{\circ}{f}$ supported on $\stackrel{\circ}{I}^{\infty}$ such that $\nu\left(\partial \mathcal{D}_{1}\right)=0$. This assumption excludes $\nu=\delta_{0}$ and $\nu=\delta_{1}$, the point masses at 0 and 1 , respectively. Since $\mathcal{D}_{1}$ is a generating partition for $f$, the Shannon-McMillan-Breiman Theorem yields,

$$
\lim _{n \rightarrow \infty}-\frac{1}{n} \log \nu\left(D_{n}(x)\right)=h_{\nu}(f)
$$

for $\nu$-a.e. $x$.

By Lemma 3.1, conformality and the fact that $f^{n}\left(D_{n}(x)\right)=[0,1)$, we have

$$
m_{t}\left(D_{n}(x)\right) \geqslant m_{t}\left(f^{n}\left(D_{n}(x)\right)\right) e^{t S_{n} \phi(x)-n\left(p(t)+V_{n}\right)}=e^{t S_{n} \phi(x)-n\left(p(t)+V_{n}\right)} .
$$

Now for $\varepsilon>0$, define

$$
G_{\varepsilon, n}:=\left\{x \in \stackrel{\circ}{I}^{\infty}: \frac{1}{n} S_{n} t \phi(x) \geqslant t \int \phi d \nu-\varepsilon \text { and } \nu\left(D_{n}(x)\right) \leqslant e^{-n\left(h_{\nu}-\varepsilon\right)}\right\} .
$$


By the ergodic theorem and the Shannon-McMillan-Breiman theorem, for $\sigma>0$ we may choose $n$ so large that $\nu\left(G_{\varepsilon, n}\right)>1-\sigma$ for all $n$ sufficiently large.

By choice of $H$, for $n>\hat{N}_{0}$ and $D \in \mathcal{D}_{n}$, if $\stackrel{\circ}{I}^{\infty} \cap D \neq 0$, then $D \subset \stackrel{\circ}{I}^{n-\hat{N}_{0}-1}$. Let $\mathcal{K}_{n}=\{D \in$ $\left.\mathcal{D}_{n}: D \cap G_{\varepsilon, n} \neq \emptyset\right\}$. Note that by construction we must have $\nu\left(\cup_{D \in \mathcal{K}_{n}} D\right) \geqslant 1-\sigma$ for sufficiently large $n$. Thus by definition of $G_{\varepsilon, n}$, the cardinality of $\mathcal{K}_{n}$ must be at least $(1-\sigma) e^{n\left(h_{\nu}-\varepsilon\right)}$. These considerations together with (3.1) yield,

$$
m_{t}\left(\stackrel{i}{I}^{n-\hat{N}_{0}-1}\right) \geqslant \sum_{D \in \mathcal{K}_{n}} m_{t}(D) \geqslant \sum_{D \in \mathcal{K}_{n}} e^{n\left(\int t \phi d \nu-p(t)-\varepsilon\right)} \geqslant(1-\sigma) e^{n\left(h_{\nu}+t \int \phi d \nu-p(t)-2 \varepsilon\right)} .
$$

Now taking logs and dividing by $n$ yields, $\log \underline{\lambda}_{t} \geqslant h_{\nu}+t \int \phi d \nu-p(t)-2 \varepsilon$, and since this is true for each $\varepsilon>0$, we conclude $\log \underline{\lambda}_{t} \geqslant h_{\nu}+t \int \phi d \nu-p(t)$.

We treat the case $\nu=\delta_{0}$ separately. In this case $P_{\delta_{0}}\left(t \phi^{H}\right)=0$, so the required inequality will hold if $\log \underline{\lambda}_{t} \geqslant-p(t)$. This is immediate since $\stackrel{i}{ }^{n} \supset\left[0, a_{n}\right)$ for $n$ sufficiently large. Thus

$$
m_{t}\left(\check{I}^{n}\right) \geqslant m_{t}\left(\left[0, a_{n}\right)\right) \geqslant C \sum_{i \geqslant n}\left|J_{i}\right|^{t} e^{-i p(t)} \geqslant C^{\prime} n^{t / \gamma} e^{-n p(t)}
$$

and $\log \underline{\lambda}_{t} \geqslant-p(t)$ follows.

The only other ergodic invariant measure which gives positive mass to $\partial \mathcal{D}_{1}$ is $\delta_{1}$, the point mass at 1. Clearly $P_{\delta_{1}}\left(t \phi^{H}\right)=-t \log 2$ and so by our previous work,

$$
\log \underline{\lambda}_{t} \geqslant-p(t) \geqslant-t \log 2-p(t)=P_{\delta_{1}}\left(t \phi^{H}\right)-p(t) .
$$

We have shown that

$$
\log \underline{\lambda}_{t} \geqslant \sup \left\{h_{\nu}(f)+t \int \phi^{H} d \nu: \nu \text { is } f \text {-invariant and ergodic and } \nu(H)=0\right\}-p(t),
$$

which is precisely what is required for the proposition.

\section{Proofs of Theorems 1.7 and 1.9 and Corollary 1.8}

In this section we prove results in the uniformly hyperbolic regime: for $t \in\left[0, t^{H}\right)$, the exponential tail decays faster than the rate of escape.

We will prove Theorem 1.7 by using the induced map $F: Y \circlearrowleft$, and an associated object known as a Young tower. We begin by recalling some basics about Young towers.

4.1. Defining the Young Tower. Given the inducing scheme with a hole, $(Y, F, \tau, H)$, we define the corresponding Young tower as follows. Recall the finite partition $\mathcal{Q}$ of images in $Y$ on which $\stackrel{\circ}{F}$ is transitive on elements defined in Section 1.2 . Define $\Delta_{0}=X$ and denote by $\Delta_{0, i}$, the finitely many elements of $\mathcal{Q}$ comprising $X$. Let

$$
\Delta=\left\{(x, n) \in \Delta_{0} \times \mathbb{N} \mid n<\tau(x)\right\} .
$$

$\Delta$ is viewed schematically as a tower with $\Delta_{\ell}=\left.\Delta\right|_{n=\ell}$ as the $\ell$ th level of the tower. The tower map, $f_{\Delta}$, is defined by $f_{\Delta}(x, \ell)=(x, \ell+1)$ if $\ell+1<\tau(x)$ and either $f^{\tau}(x) \in H$, in which case we define a hole $H_{\tau, j} \subset \Delta_{\tau}$, or $f^{\tau}(x) \in \Delta_{0}$, in which case $f_{\Delta}(x, \tau(x)-1)=\left(f^{\tau}(x), 0\right)=(F(x), 0)$. There is a canonical projection $\pi: \Delta \rightarrow I$ satisfying $\pi \circ f_{\Delta}=f \circ \pi$. $\Delta_{0}$ is identified with $\cup_{Q \in \mathcal{Q}^{\prime}} Q$ so that $\left.\pi\right|_{\Delta_{0}}=I d$. 
Let $\left\{X_{i}\right\}$ denote the maximal partition of $\Delta_{0}=X$ into intervals such that $\tau_{Y}$ is constant on each $X_{i}$. The partition $\left\{X_{i}\right\}$ then induces a countable Markov partition $\left\{\Delta_{\ell, j}\right\}$ on $\Delta$ via the identification $\Delta_{\ell, j}=f_{\Delta}^{\ell}\left(X_{j}\right)$, for $0<\ell<\tau\left(X_{j}\right)$. On level $\ell=0$, we insist on keeping the partition finite and use $\Delta_{0, i}$ as our partition elements. Since $f$ is expanding, the partition $\left\{X_{i}\right\}$, and hence the partition $\left\{\Delta_{\ell, j}\right\}$, is generating.

Note that since $H$ is a 1 -cylinder in $\mathcal{P}_{N_{0}}$, by definition of $\left\{X_{i}\right\}, \tilde{H}:=\pi^{-1} H$ is the union of countably many partition elements $\Delta_{\ell, j}$. We set $\stackrel{\AA}{\Delta}=\Delta \backslash \tilde{H}$ and refer to the corresponding partition elements as $\stackrel{\circ}{\Delta}_{\ell, j}$. Similarly, we define $\stackrel{\circ}{\Delta}^{n}=\bigcap_{i=0}^{n} f_{\Delta}^{-i} \stackrel{\circ}{\Delta}$ and $\stackrel{\circ}{\Delta}_{\Delta}^{n}=\left.f_{\Delta}^{n}\right|_{\Delta^{n}}, n \in \mathbb{N}$.

Given a potential $\varphi$ and a $\varphi$-conformal reference measure $m$ on $I$, we define a reference measure $\bar{m}$ on $\Delta$ by $\bar{m}=m$ on $\Delta_{0}$ and $\left.\bar{m}\right|_{\Delta_{\ell}}=\left.\left(f_{\Delta}\right)_{*} \bar{m}\right|_{\Delta_{\ell-1} \cap f_{\Delta}^{-1} \Delta_{\ell}}$ for $\ell \geqslant 1$. For $x \in \Delta_{\ell}$, let $x^{-}:=f^{-\ell} x$ denote the pullback of $x$ to $\Delta_{0}$. We define the induced potential on $\Delta$ by

$$
\varphi_{\Delta}(x)=S_{\tau} \varphi\left(x^{-}\right) \text {for } x \in f_{\Delta}^{-1}\left(\Delta_{0}\right) \text { and } \varphi_{\Delta}=0 \text { on } \Delta \backslash f_{\Delta}^{-1}\left(\Delta_{0}\right) .
$$

With these definitions, the measure $\bar{m}$ is $\varphi_{\Delta}$-conformal.

Lemma 4.1. For $t \in[0,1)$, let $\bar{m}_{t}$ be the measure on $\stackrel{\AA}{\Delta}$ induced by $m_{t}$, the conformal measure for the potential $t \phi-p(t)$. There exists $C>0$, independent of $t$, such that for $n \geqslant 0, \bar{m}_{t}\left(\Delta_{n}\right) \leqslant$ $C \frac{1}{p(t)} n^{-t\left(1+\frac{1}{\gamma}\right)} e^{-n p(t)}$.

Proof. This follows immediately from Lemma 1.1 and the definition of $\bar{m}_{t}$ since $m_{t}(\tau=n) \approx$ $n^{-t\left(1+\frac{1}{\gamma}\right)} e^{-n p(t)}$ due to conformality and the growth in $D f^{n}$ given by (D1) of Section 4.3 .

We define the transfer operator ${\stackrel{\circ}{\mathcal{L}_{\varphi_{\Delta}^{H}}}}$ associated with the punctured potential $\varphi_{\Delta}^{H}$ and acting on $L^{1}(\bar{m})$ by

$$
{\stackrel{\circ}{\mathcal{L}_{\varphi_{\Delta}^{H}}^{n}}}^{n} \psi(x)=\sum_{f_{\Delta}^{n} y=x} \psi(x) e^{S_{n} \varphi_{\Delta}(y)} 1_{\Delta^{n}}(y)=\mathcal{L}_{\varphi_{\Delta}}^{n}\left(\psi 1_{\Delta^{n}}\right)(x) .
$$

With these definitions, $\mathcal{L}$ satisfies the following change of variable formula,

$$
\int_{\dot{\Delta}} \dot{\mathcal{L}}_{\varphi_{\Delta}^{H}}^{n} \psi d \bar{m}=\int_{\grave{\Delta}^{n}} \psi d \bar{m}
$$

which in turn links the spectral properties of $\stackrel{\circ}{\mathcal{L}}_{\varphi_{\Delta}^{H}}$ to the escape rate with respect to $\bar{m}$.

In order to translate between densities on $I$ and on $\Delta$, for $\tilde{\psi} \in L^{1}(\bar{m})$ on $\Delta$, define the projection

$$
P_{\pi, m} \tilde{\psi}(x)=\sum_{y \in \pi^{-1}(x)} \frac{\tilde{\psi}(y)}{J_{m} \pi(y)},
$$

where $J_{m} \pi$ is the Jacobian of $\pi$ with respect to the measures $m$ and $\bar{m}$. Then $P_{\pi, m} \tilde{\psi} \in L^{1}(m)$ and

$$
\stackrel{\circ}{\mathcal{L}}_{\varphi^{H}}^{n}\left(P_{\pi, m} \tilde{\psi}\right)=P_{\pi, m}\left(\stackrel{\circ}{\mathcal{L}}_{\varphi_{\Delta}^{H}}^{n} \tilde{\psi}\right) .
$$

Indeed, the following lemma shows that the escape rates from $I$ and from $\Delta$ are the same.

Lemma 4.2. Let $\bar{m}_{t}$ be the measure on $\Delta$ induced by $m_{t}$ as in Lemma 4.1. Then for each $t \in[0,1]$,

$$
\log \bar{\lambda}_{t}=\limsup _{n} \frac{1}{n} \log m_{t}\left(\stackrel{\circ}{I}^{n}\right)=\limsup _{n} \frac{1}{n} \log \bar{m}_{t}\left(\stackrel{\circ}{\Delta}^{n}\right) .
$$


Proof. When $t=1, p(t)=0$ and the above quantities are all 0 , so equality is trivial. Now assume $t<1$.

Recall the finite partition $\mathcal{Q}$ of $[1 / 2,1] \backslash H$ defined in Section 1.2 on which $\stackrel{\circ}{F}$ is transitive on elements. It follows from the definition of non-swallowing that $\stackrel{\circ}{\perp} \subset \cup_{i \geqslant 0} f^{i}\left(\cup_{Q \in \mathcal{Q}} Q\right)=\pi(\stackrel{\Delta}{\Delta})$ up to a countable collection of points comprising the pre-images of 0 . Thus

$$
\log \bar{\lambda}_{t}=\limsup _{n} \frac{1}{n} \log m_{t}\left(\stackrel{\circ}{I}^{n}\right)=\limsup _{n} \frac{1}{n} \log m_{t}\left(\stackrel{\circ}{I}^{n} \cap \pi(\stackrel{\circ}{\Delta})\right) .
$$

Due to the transitivity and finitely many elements of $\mathcal{Q}$, we may choose a collection of indices, $\mathcal{K}:=\{(\ell, j)\}$ with $\ell \leqslant L$ for some $L>0$ such that $\pi\left(\cup_{(\ell, j) \in \mathcal{K}} \Delta_{\ell, j}\right)=\pi(\stackrel{\circ}{\Delta})$ and $\pi\left(\Delta_{\ell, j}\right) \cap \pi\left(\Delta_{\ell^{\prime}, j^{\prime}}\right)=\emptyset$ for all pairs $(\ell, j) \neq\left(\ell^{\prime}, j^{\prime}\right)$ in $\mathcal{K}$. Further, we may choose $\mathcal{K}$ so that all elements of the base, $\Delta_{0, i}$, belong to $\mathcal{K}$.

Now let $P_{\pi, t}$ be the projection defined by 4.2 with respect to the measures $m_{t}$ and $\bar{m}_{t}$. Denote by $J_{t} \pi$ the relevant Jacobian. On each $\Delta_{\ell, j}$ for $(\ell, j) \in \mathcal{K}$, define $\tilde{\psi}(x)=J_{t} \pi(x)$. Set $\tilde{\psi} \equiv 0$ elsewhere on $\stackrel{\Delta}{\Delta}$. Then by construction of $\mathcal{K}, P_{\pi, t} \tilde{\psi}=1_{\pi(\Delta)}$.

Note that by conformality, for $x \in \Delta_{\ell}, J_{t} \pi(x)=e^{-t S_{\ell} \phi(x)+\ell p(t)}$. Thus there exists $M>0$, depending on $L$, such that $1 \leqslant \tilde{\psi} \leqslant M$ on $\Delta$. Also, $J_{t} \pi=1$ on $\Delta_{0}$ so that $\tilde{\psi}=1$ on $\AA_{0}$.

Now integrating,

$$
\bar{m}_{t}\left(\stackrel{\circ}{\Delta}^{n}\right)=\int_{\dot{\Delta}^{n}} 1 d \bar{m}_{t} \geqslant \frac{1}{M} \int_{\AA^{n}} \tilde{\psi} d \bar{m}_{t}=\frac{1}{M} \int_{\pi\left(\AA^{n}\right)} P_{\pi, t} \tilde{\psi} d m_{t}=\frac{1}{M} m_{t}\left(\stackrel{\circ}{I}^{n} \cap \pi(\stackrel{\circ}{\Delta})\right),
$$

so we deduce $\lim \sup _{n} \frac{1}{n} \log m_{t}\left(\stackrel{\circ}{I}^{n}\right) \leqslant \lim \sup _{n} \frac{1}{n} \log \bar{m}\left(\stackrel{\circ}{\Delta}^{n}\right)$, using (4.3).

On the other hand, notice that since $I^{n} \supset\left[0, a_{n+h}\right]$, where $h$ denotes the maximal index such that $J_{h} \cap H \neq \emptyset$, we have $\bar{\lambda}_{t} \geqslant e^{-p(t)}$. Thus by Lemma 4.1 ,

$$
\begin{aligned}
& \bar{m}_{t}\left(\AA^{n}\right)=\int_{\grave{\Delta}^{n} \cap(\tau \leqslant n)} d \bar{m}_{t}+\int_{\dot{\Delta}^{n} \cap(\tau>n)} d \bar{m}_{t} \leqslant n \int_{\grave{\Delta}^{n} \cap \Delta_{0}} d \bar{m}_{t}+C e^{-p(t) n} \\
& \leqslant n \int_{\AA^{n}} \tilde{\psi} d \bar{m}_{t}+C e^{-p(t) n} \leqslant n m_{t}\left(\stackrel{\circ}{I}^{n}\right)+C e^{-p(t) n} .
\end{aligned}
$$

Since as noted above, $\bar{\lambda}_{t} \geqslant e^{-p(t)}$, we conclude that $\lim \sup _{n} \frac{1}{n} \log \bar{m}_{t}\left(\stackrel{\circ}{\Delta}^{n}\right) \leqslant \lim \sup _{n} \frac{1}{n} \log m_{t}\left(\stackrel{\circ}{I}^{n}\right)$.

4.2. Abstract Results for Young Towers with Holes. In this section, we prove results about an abstract Young tower with holes which may be of independent interest: We prove the transfer operator on the tower has a spectral gap in a space of Holder continuous functions under the assumption that the escape rate is slower than the decay rate in the levels of the tower. After stating our assumptions formally below, we will describe how the present results generalize the existing results of $[\mathrm{BDM}]$ and related references.

We assume that we have a tower map $f_{\Delta}: \Delta \circlearrowleft$ with a countable generating Markov partition $\left\{\Delta_{\ell, j}\right\}$ and such that the return map to the base has finitely many images, denoted by $\Delta_{0, i}$. We further assume that our reference measure $\bar{m}$ is conformal with respect to a potential $\varphi_{\Delta}$.

In this context, we assume the following properties of the tower map.

(P1) (Exponential tail.) There exist constants $C, \alpha>0$ such that $\bar{m}\left(\Delta_{n}\right) \leqslant C e^{-\alpha n}$, for $n \in \mathbb{N}$. 
We define a natural metric adapted to the dynamics as follows. Let $\tau^{n}(x)$ be the time of the $n$th return of $x$ to $\Delta_{0}$. Define the separation time on $\Delta$ to be

$$
s(x, y)=\min \left\{n \geq 0: f_{\Delta}^{\tau^{n}}(x), f_{\Delta}^{\tau^{n}}(y) \text { lie in different partition elements } \Delta_{0, i}\right\} .
$$

$s(x, y)$ is finite $\bar{m}$-almost everywhere since $\left\{\Delta_{\ell, j}\right\}$ is a generating partition for $f^{\tau}$. Choos $\biguplus^{4} \delta>0$ and define a metric $d_{\delta}$ on $\Delta$ by $d_{\delta}(x, y)=e^{-\delta s(x, y)}$.

We introduce a hole $H$ in $\Delta$ which is the union of countably many partition elements $\Delta_{\ell, j}$, i.e. $H=\cup_{\ell, k} H_{\ell, k}$ where $H_{\ell, k}=\Delta_{\ell, j}$ for some $j$. Set $H_{\ell}=\cup_{j} H_{\ell, j} \subset \Delta_{\ell}$. For simplicity we assume that the base $\Delta_{0}$ contains no holes (this can always be arranged in the construction of the tower by choosing a suitable reference set $X$ ). We assume that $f_{\Delta}^{\circ}$ is transitive and aperiodic on the elements $\left\{\Delta_{0, i}\right\}$ after the introduction of the hole.

(P2) (Slow escape.) Define $\log \bar{\lambda}=\limsup _{n \rightarrow \infty} \frac{1}{n} \log \bar{m}\left(\stackrel{\circ}{\Delta}^{n}\right)$. We assume that $-\log \lambda<\alpha$.

(P3) (Bounded distortion.) We suppose that $e^{\varphi_{\Delta}}$ is Lipschitz in the metric $d_{\delta}$. Furthermore, we assume there exits $C_{d}>0$ such that for all $x, y \in \Delta$ and $n \geq 0$,

$$
\left|e^{S_{n} \varphi_{\Delta}(x)-S_{n} \varphi_{\Delta}(y)}-1\right| \leqslant C_{d} d_{\delta}\left(f_{\Delta}^{n} x, f_{\Delta}^{n} y\right) .
$$

(P4) (Subexponential growth of potential) For each $\varepsilon>0$, there exists $C>0$, such that

$$
\left|S_{\tau} \varphi_{\Delta}(x)\right| \leqslant C e^{\varepsilon \tau(x)} \quad \text { at first return times } \tau \text { for all } x \in \Delta_{0} .
$$

A spectral gap for tower maps with holes was established in [BDM] (see also [D1, D2, DWY1, DWY2 for applications) under stronger conditions than those listed here.

Remark 4.3. There are two significant differences between our assumptions and those in [BDM]. (1) The metric $d_{\delta}$ in $[\mathrm{BDM}]$ uses a stronger notion of separation time which requires the derivative of the underlying map $f$ to grow exponentially at return times; since our maps have only polynomial growth in the derivative, we adopt a weaker metric which requires significant changes to our function space arguments; in particular, see the proofs of Lemmas 4.5 and 4.10 . (2) The assumption in [BDM] on the size of the hole is comparatively strict, allowing one to control the maximum amount of mass lost in a single iterate of the map; by contrast, our assumption (P2) only assumes that the tail decay is faster than the escape rate asymptotically, which again requires significant revisions to the proof of the spectral gap.

Choose $\beta$ satisfying $-\log \bar{\lambda}<\beta<\alpha$. We define the standard weighted $L^{\infty}$-norm on the space of functions on $\Delta$, given by

$$
\|\psi\|_{\infty}=\sup _{\ell} \sup \left\{e^{-\beta \ell}|\psi(x)|: x \in \Delta_{\ell}\right\}
$$

along with a Lipschitz norm

$$
|\psi|_{L i p}=\sup _{\ell} e^{-\beta \ell} \sup \left\{e^{-\delta s(x, y)}|\psi(x)-\psi(y)|: x, y \in \Delta_{\ell}\right\}
$$

We define a Banach space $\left(\mathcal{B},\|\cdot\|_{\mathcal{B}}\right)$ where $\|\psi\|_{\mathcal{B}}=|\psi|_{\text {Lip }}+\|\psi\|_{\infty}$.

We proceed to prove the quasi-compactness for the punctured transfer operator $\stackrel{\circ}{\mathcal{L}}_{\Delta}:=\dot{\mathcal{L}}_{\varphi_{\Delta}^{H}}$ (i.e., with punctured potential $\left.\varphi_{\Delta}^{H}\right)$ acting on $\mathcal{B}$ under assumptions $(\mathrm{P} 1)-(\mathrm{P} 4)$.

\footnotetext{
${ }^{4}$ In this abstract setting, the choice of $\delta>0$ is constrained only by (P3); however, in applications, $\delta$ will be constrained by the expansion and regularity of the underlying map $f$. We will introduce this restriction on $\delta$ when we apply this abstract framework to our map with neutral fixed point in Section 4.3 .
} 
To do this, let $1_{\beta}$ denote the function which takes constant value $e^{\beta \ell}$ on $\Delta_{\ell}$. Note $1_{\beta} \in \mathcal{B}$ since $\left\|1_{\beta}\right\|_{\mathcal{B}}=1$. Define

$$
\bar{\lambda}_{\beta}=\limsup _{n} \frac{1}{n} \log \int_{\grave{\Delta}^{n}} 1_{\beta} d \bar{m},
$$

and note that $\bar{\lambda}_{\beta} \geqslant \bar{\lambda}>e^{-\alpha}$ by $(\mathrm{P} 2)$.

Lemma 4.4. The spectral radius of $\dot{\mathcal{L}}_{\Delta}$ on $\mathcal{B}$ is at least $\bar{\lambda}_{\beta}$.

Proof. Note

$$
\int_{\dot{\Delta}^{n}} 1_{\beta} d \bar{m}=\int \dot{\mathcal{L}}_{\Delta}^{n}\left(1_{\beta}\right) d \bar{m} \leqslant \sum_{\ell} e^{\beta \ell} \bar{m}\left(\stackrel{\Delta}{\ell}_{\ell}\right)\left\|\dot{\mathcal{L}}_{\Delta}^{n}\left(1_{\beta}\right)\right\|_{\infty} \leqslant \sum_{\ell} C e^{(\beta-\alpha) \ell}\left\|\dot{\mathcal{L}}_{\Delta}^{n}\right\|\left\|1_{\beta}\right\|_{\mathcal{B}} \leqslant C^{\prime}\left\|\dot{\mathcal{L}}_{\Delta}^{n}\right\| .
$$

Thus $\lim \sup _{n} \frac{1}{n} \log \left\|\dot{\mathcal{L}}_{\Delta}^{n}\right\| \geqslant \lim \sup _{n} \frac{1}{n} \log \int_{\grave{\Delta}^{n}} 1_{\beta} d \bar{m}=\log \bar{\lambda}_{\beta}$ as required.

Next we prove that the essential spectral radius is strictly smaller than $\bar{\lambda}_{\beta}$. This bound, together with the preceding lemma, will imply that $\stackrel{\circ}{\mathcal{L}}_{\Delta}$ is quasi-compact as an operator on $\mathcal{B}$.

Lemma 4.5 (Lasota-Yorke Inequality). There exists $\sigma<\bar{\lambda}_{\beta}$ and $C>0$ such that for all $\psi \in \mathcal{B}$ and $n \geqslant 0$,

$$
\left\|\stackrel{\circ}{\mathcal{L}}_{\Delta}^{n} \psi\right\|_{\mathcal{B}} \leqslant C \sigma^{n}\|\psi\|_{\mathcal{B}}+C|\psi|_{L^{1}(\bar{m})} .
$$

Proof. Fix $\psi \in \mathcal{B}$ and $n \geqslant 0$.

Step 1. For $\ell \geqslant n$ and $x \in \Delta_{\ell}$, we estimate

$$
\left|\stackrel{\circ}{\mathcal{L}}_{\Delta}^{n} \psi(x)\right|=\left|\psi\left(f_{\Delta}^{-n} x\right)\right| \leqslant e^{\beta(\ell-n)}\|\psi\|_{\infty} .
$$

Thus $\left\|\left.\dot{\mathcal{L}}_{\Delta}^{n} \psi\right|_{\Delta_{\ell}}\right\|_{\infty} \leqslant e^{-\beta n}\|\psi\|_{\infty}$. Similarly, for $x, y \in \Delta_{\ell}$

$$
e^{-\delta s(x, y)}\left|\dot{\mathcal{L}}_{\Delta}^{n} \psi(x)-\stackrel{\circ}{\mathcal{L}}_{\Delta}^{n} \psi(y)\right|=e^{-\delta s\left(f_{\Delta}^{-n}(x), f_{\Delta}^{-n}(y)\right)}\left|\psi\left(f_{\Delta}^{-n} x\right)-\psi\left(f_{\Delta}^{-n} y\right)\right|,
$$

since the separation time for $x, y$ is the same as that for $f_{\Delta}^{-n} x$ and $f_{\Delta}^{-n} y$. Thus $\left\|\left.\dot{\mathcal{L}}_{\Delta}^{n} \psi\right|_{\Delta_{\ell}}\right\|_{L i p} \leqslant$ $e^{-\beta n}\|\psi\|_{\text {Lip }}$.

Step 2. Now let $x \in \Delta_{0}$. We have

$$
\dot{\mathcal{L}}_{\Delta}^{n} \psi(x)=\sum_{u \in \dot{f}_{\Delta}^{-n}(x)} \psi(u) e^{t S_{n} \varphi_{\Delta}^{H}(u)}=\sum_{u \in \dot{f}_{\Delta}^{-n}(x)}(\psi(u)-\psi(v)) e^{t S_{n} \varphi_{\Delta}^{H}(u)}+\psi(v) e^{t S_{n} \varphi_{\Delta}^{H}(u)}
$$

where $E_{n}(u)$ is the $n$-cylinder containing $u$ and $v \in E_{n}(u)$ satisfies $\psi(v)=\bar{m}\left(E_{n}\right)^{-1} \int_{E_{n}} \psi d \bar{m}$.

Due to bounded distortion of $\varphi_{\Delta}$ given by (P2) and the fact that $f_{\Delta}^{n}\left(E_{n}\right)=\Delta_{0, i}$ for some $i \geqslant 0$, the second term in the above sum is bounded by $C \bar{m}\left(\Delta_{0, i}\right)^{-1} \int_{E_{n}(u)} \psi d \bar{m}$ and summing over $u$ yields the bound $C^{\prime} \int_{\Delta^{n}} \psi d \bar{m}$.

To estimate the first term, we split it into two parts: For $T>0$, let $A_{n, T}$ denote the set of points in $\stackrel{\circ}{\Delta}^{n} \cap \stackrel{\circ}{f}_{\Delta}^{-n}\left(\Delta_{0}\right)$ that make at least $n / T$ returns to $\Delta_{0}$ by time $n$, and let $A_{n, T}^{c}=\left(\stackrel{\circ}{\Delta}^{n} \cap \stackrel{\circ}{\Delta}_{\Delta}^{-n}\left(\Delta_{0}\right)\right) \backslash A_{n, T}$. 
Then letting $\ell(u)$ denote the level containing $u$,

$$
\begin{aligned}
& \sum_{u \in f_{\Delta}^{-n}(x)}(\psi(u)-\psi(v)) e^{t S_{n} \varphi_{\Delta}^{H}(u)} \\
= & \sum_{\substack{u \in f_{\Delta}^{-n}(x) \\
u \in A_{n, T}}}(\psi(u)-\psi(v)) e^{t S_{n} \varphi_{\Delta}^{H}(u)}+\sum_{\substack{u \in f_{\Delta}^{-n}(x) \\
u \in A_{n, T}^{c}}}(\psi(u)-\psi(v)) e^{t S_{n} \varphi_{\Delta}^{H}(u)} \\
\leqslant & \sum_{\substack{u \in f_{\Delta}^{-n}(x) \\
u \in A_{n, T}}} C e^{-\delta n / T}\|\psi\|_{L i p} e^{\beta \ell(u)} \bar{m}\left(E_{n}(u)\right)+\sum_{\substack{u \in f_{\Delta}^{-n}(x) \\
u \in A_{n, T}^{c}}} C\|\psi\|_{L i p} e^{\beta \ell(u)} \bar{m}\left(E_{n}(u)\right) \\
\leqslant & C e^{-\delta n / T}\|\psi\|_{L i p} \int_{\dot{\Delta}^{n}} 1_{\beta} d \bar{m}+C\|\psi\|_{L i p} \int_{\dot{\Delta}^{n} \cap f_{\Delta}^{-n}\left(\Delta_{0}\right) \cap A_{n, T}^{c}} 1_{\beta} d \bar{m} .
\end{aligned}
$$

This first term above clearly contracts at an exponential rate $e^{-\delta n / T} \int_{\AA^{n}} 1_{\beta} d \bar{m}<\bar{\lambda}_{\beta}^{n}$. Thus it remains to prove the contraction in the second term is sufficiently fast.

We associate to each $x \in \AA_{\Delta}^{-n}\left(\Delta_{0}\right) \cap A_{n, T}^{c}$ a sequence of times $r_{1}, \ldots r_{s}$, such that $f_{\Delta}^{r_{i}}(x) \in \Delta_{0}$ for each $i$; it follows from the definition of $A_{n, T}^{c}$ that $s \leqslant n / T-1$. Note also that $\sum_{i=1}^{s} r_{i}=n$ since $x \in f_{\Delta}^{-n}\left(\Delta_{0}\right)$. However, each connected component in $f_{\Delta}^{-n}\left(\Delta_{0}\right) \cap A_{n, T}^{c}$ on level $\Delta_{\ell}$, can be uniquely associated with a connected component in $\Delta_{0}$ such that each $y=f_{\Delta}^{-\ell}(x)$ in this interval is associated with the sequence of return times $r_{1}, \ldots, r_{s}$ such that $\sum_{i=1}^{s} r_{i}=n+\ell$. Now

$$
\text { \# }\left\{s \text {-tuples with } \sum_{i=1}^{s} r_{i}=n+\ell\right\}=\left(\begin{array}{c}
n+\ell-1 \\
s-1
\end{array}\right) \leqslant\left(\begin{array}{c}
n+\ell-1 \\
\frac{n}{T}-1
\end{array}\right) \leqslant C\left(1+\eta_{T}\right)^{n+\ell},
$$

where $\eta_{T} \rightarrow 0$ as $T \rightarrow \infty$. Also, at each return, $\bar{m}\left(\tau=r_{i}\right) \leqslant C_{0} e^{-\alpha r_{i}}$. So conditioning $s$ times, we have

$$
\bar{m}\left(A_{n, T} \cap \stackrel{\circ}{\Delta}_{\ell} \cap \stackrel{\circ}{f}_{\Delta}^{-n}\left(\Delta_{0}\right)\right) \leqslant \sum_{s=1}^{\frac{n}{T}-1} \sum_{\text {relevant } s \text {-tuples }} C_{0}^{s} e^{-(n+\ell) \alpha} \leqslant C C_{0}^{n / T}\left(1+\eta_{T}\right)^{n+\ell} e^{-(n+\ell) \alpha} .
$$

Fix $0<\varepsilon<\min \left\{\alpha-\beta, \alpha+\log \bar{\lambda}_{\beta}\right\}$. Then choose $T$ sufficiently large that $\left(1+\eta_{T}\right) C_{0}^{1 / T} \leqslant e^{\varepsilon}$. Using (4.8), we have the contraction in the second term at the end of 4.7) bounded by

$$
\int_{\grave{\Delta}^{n} \cap f_{\Delta}^{-n}\left(\Delta_{0}\right) \cap A_{n, T}^{c}} 1_{\beta} d \bar{m} \leqslant \sum_{\ell \geqslant 0} C e^{\beta \ell} e^{-(n+\ell)(\alpha-\varepsilon)} \leqslant C e^{-n(\alpha-\varepsilon)} .
$$

Putting this estimate together with (4.7), we have

$$
\left|\stackrel{\circ}{\mathcal{L}}_{\Delta}^{n} \psi(x)\right| \leqslant C \sigma^{n}\|\psi\|_{L i p}+C \int_{\check{\Delta}^{n}} \psi d \bar{m}
$$

for all $x \in \Delta_{0}$ and some constant $\sigma<\bar{\lambda}_{\beta}$.

Now for the bound on the Lipschitz norm, we estimate similarly for $x, y \in \Delta_{0}$,

$$
\begin{aligned}
\left|\dot{\mathcal{L}}_{\Delta}^{n} \psi(x)-\dot{\mathcal{L}}_{\Delta}^{n} \psi(y)\right| & \leqslant \sum_{\substack{u \in f_{\Delta}^{n}(x) \\
v \in \dot{f}_{\Delta}^{-n}(y)}}|\psi(u)-\psi(v)| e^{S_{n} \varphi_{\Delta}(u)}+|\psi(v)|\left|e^{S_{n} \varphi_{\Delta}(u)}-e^{S_{n} \varphi_{\Delta}(v)}\right| \\
& \leqslant \sum_{u, v}\|\psi\|_{L i p} e^{-\delta s(u, v)} e^{\beta \ell(u)} e^{S_{n} \varphi_{\Delta}(u)}+C e^{-\delta s(x, y)}|\psi(v)| e^{S_{n} \varphi_{\Delta}(u)},
\end{aligned}
$$


where we have used bounded distortion in the last line. Notice that once we divide by $e^{-\delta s(x, y)}$, this is precisely the same expression which had to be estimated in (4.6) and so is also bounded by 4.10. Thus

$$
\left\|\left.\stackrel{\circ}{\mathcal{L}}_{\Delta}^{n} \psi\right|_{\Delta_{0}}\right\|_{L i p} \leqslant C \sigma^{n}\|\psi\|_{L i p}+C \int_{\dot{\Delta}^{n}} \psi d \bar{m}
$$

Step 3. We complete the proof of the proposition by taking $x, y \in \Delta_{\ell}, \ell<n$, using Step 1 to estimate the first $\ell$ steps from $\Delta_{\ell}$ to $\Delta_{0}$, and then Step 2 to estimate the remaining $n-\ell$ steps:

$$
\left\|\left.\stackrel{\mathscr{L}}{\Delta}_{\Delta}^{n} \psi\right|_{\Delta_{\ell}}\right\|_{\infty} \leqslant e^{-\beta \ell}\left\|\left.\stackrel{\mathcal{L}}{\Delta}_{\Delta}^{n-\ell} \psi\right|_{\Delta_{0}}\right\|_{\infty} \leqslant C e^{-\beta \ell} \sigma^{n-\ell}\|\psi\|_{\mathcal{B}}+C|\psi|_{L^{1}} .
$$

A similar estimate holds for $\left\|\mathcal{L}_{\Delta}^{n} \psi\right\|_{L i p}$, completing the proof of the lemma.

Since the unit ball of $\mathcal{B}$ is compactly embedded in $L^{1}(\bar{m})$, it follows from the by-now classical results $[\mathrm{HH}]$ together with Lemma 4.5 that $\stackrel{\circ}{\mathcal{L}}_{\Delta}$ is quasi-compact as an operator on $\mathcal{B}$ : Its essential spectral radius is bounded by $\sigma<\bar{\lambda}$, and for any $\sigma^{\prime}>\sigma$, its spectrum outside the disk of radius $\sigma^{\prime}$ comprises only finitely many eigenvalues each of finite multiplicity. It follows from Lemma 4.4 . that the spectral radius of $\dot{\mathcal{L}}_{\Delta}$ is at least $\bar{\lambda}>\sigma$ so that the peripheral spectrum is nonempty and lies outside the disk of radius $\sigma$.

Our next step in the proof is showing that $\stackrel{\circ}{\mathcal{L}}_{\Delta}$ has a real eigenvalue greater than $\sigma$ and a corresponding eigenvector which is strictly positive. Once this is done, we will use it to prove that $\stackrel{\circ}{\mathcal{L}}_{\Delta}$ has a spectral gap.

Lemma 4.6. There exists $M_{0}>0$ such that $\left\|\frac{\dot{\mathcal{L}}_{\Delta}^{n} 1_{\beta}}{\left|\dot{\mathcal{L}}_{\Delta}^{n} 1_{\beta}\right|_{1}}\right\|_{\mathcal{B}} \leqslant M_{0}$ for all $n \geqslant 0$. Moreover, the escape rate $-\log \lambda_{\beta}$ with respect to $1_{\beta} \bar{m}$ exists and $\lambda_{\beta}=\underline{\lambda}_{\beta}=\bar{\lambda}_{\beta}$ is the spectral radius of $\dot{\mathcal{L}}_{\Delta}$ on $\mathcal{B}$.

Proof. Let $\rho,|\rho|>\sigma$, be an eigenvalue of maximum modulus of $\dot{\mathcal{L}}_{\Delta}$. Since the peripheral spectrum of $\dot{\mathcal{L}}_{\Delta}$ is finite dimensional, we may choose $\rho$ such that $\rho$ has maximum defect for eigenvalues on the circle of radius $|\rho|$. Thus there exists $d \geqslant 1$ and $g_{i} \in \mathcal{B}, g_{i} \neq 0, i=1, \ldots, d$, such that $\left(\stackrel{\mathcal{L}}{\Delta}_{\Delta}-\rho I\right) g_{i}=g_{i-1}$ for $i>1$ and $\left(\dot{\mathcal{L}}_{\Delta}-\rho I\right) g_{1}=0$, and $d$ is the maximal such index for eigenvalues with modulus $|\rho|$. Thus for $n>d$, we have

$$
\stackrel{\mathcal{L}}{\Delta}_{\Delta}^{n} g_{d}=\sum_{i=0}^{d-1}\left(\begin{array}{l}
n \\
i
\end{array}\right) \rho^{n-i} g_{d-i} .
$$

Integrating over $\stackrel{\circ}{\Delta}$, we use the above identity to obtain the following lower bound,

$$
\begin{aligned}
\int_{\dot{\Delta}} \dot{\mathcal{L}}_{\Delta}^{n}\left|g_{d}\right| d \bar{m} & \geqslant\left.\int_{\dot{\Delta}}|| \rho\right|^{n-d+1}\left(\begin{array}{c}
n \\
d-1
\end{array}\right)\left|g_{1}\right|-\mid \sum_{i=0}^{d-2}\left(\begin{array}{c}
n \\
i
\end{array}\right) \rho^{n-i} g_{d-i} \| d \bar{m} \\
& \geqslant \int_{\dot{\Delta}}|\rho|^{n-d+1}\left(\begin{array}{c}
n \\
d-1
\end{array}\right)\left|g_{1}\right| d \bar{m}-\int_{\Sigma}\left|\sum_{i=0}^{d-2}\left(\begin{array}{c}
n \\
i
\end{array}\right) \rho^{n-i} g_{d-i}\right| d \bar{m}
\end{aligned}
$$

Since the first term is a polynomial of degree $d-1$ while the second term has degree at most $d-2$, there exists $C_{1}>0$ and $N>0$ such that for $n \geqslant N$,

$$
\int_{\dot{\Delta}} \stackrel{\circ}{L}_{\Delta}^{n}\left|g_{d}\right| \geqslant C_{1} n^{d-1}|\rho|^{n}
$$


Thus for $n \geqslant N$,

$$
C_{1} n^{d-1}|\rho|^{n} \leqslant \int_{\dot{\Sigma}} \stackrel{\circ}{\mathcal{L}}_{\Delta}^{n}\left|g_{d}\right| d \bar{m}=\int_{\dot{\Delta}^{n}}\left|g_{d}\right| d \bar{m} \leqslant\left\|g_{d}\right\|_{\infty} \int_{\dot{\Xi}^{n}} 1_{\beta} d \bar{m}=\left\|g_{d}\right\|_{\infty} \int_{\dot{\Delta}} \stackrel{\circ}{\mathcal{L}}_{\Delta}^{n} 1_{\beta} d \bar{m} .
$$

On the other hand, using the spectral decomposition of $\dot{\mathcal{L}}_{\Delta}$ given by quasi-compactness, we have $\left\|\stackrel{\circ}{L}_{\Delta}^{n} 1_{\beta}\right\|_{\mathcal{B}} \leqslant C_{2} n^{d-1}|\rho|^{n}$ for some $C_{2}>0$ and all $n \geqslant 0$. Thus the bound on $\left\|\frac{\dot{\mathcal{L}}_{\Delta}^{n} 1_{\beta}}{\left|\dot{\mathcal{L}}_{\Delta}^{n} 1_{\beta}\right|_{1}}\right\|_{\mathcal{B}}$ follows using this together with 4.12 for $n \geqslant N$. The bound for $n<N$ is obtained by taking the maximum over the finitely many terms and noting that each term is finite since $\dot{\mathcal{L}}_{\Delta}$ is a bounded operator on $\mathcal{B}$ and $\bar{m}\left(\stackrel{\circ}{ }^{n}\right)>0$ for each $n$.

Now (4.12) implies $\underline{\lambda}_{\beta} \geqslant|\rho|$, while Lemma 4.4 implies $\bar{\lambda}_{\beta} \leqslant|\rho|$. Thus $\lambda_{\beta}$ exists and is the spectral radius of $\dot{\mathcal{L}}_{\Delta}$.

From now on, we use the notation $\lambda_{\beta}$ rather than $\bar{\lambda}_{\beta}$ since we know the escape rate with respect to $1_{\beta} \bar{m}$ exists.

Let

$$
\psi_{n}=\frac{\sum_{k=1}^{n} \lambda_{\beta}^{-k} \stackrel{\circ}{\mathcal{L}}_{\Delta}^{k} 1_{\beta}}{\sum_{k=1}^{n} \lambda_{\beta}^{-k}\left|\stackrel{\mathcal{L}}{L}_{\Delta}^{k} 1_{\beta}\right|_{1}} .
$$

Notice that $\left(\psi_{n}\right)_{n}$ is a sequence of probability densities and that $t^{5}$ by Lemma 4.6 ,

$$
\left\|\psi_{n}\right\|_{\mathcal{B}} \leqslant \frac{\sum_{k=1}^{n} \lambda_{\beta}^{-k}\left\|\dot{\mathcal{L}}_{\Delta}^{k} 1_{\beta}\right\|_{\mathcal{B}}}{\sum_{k=1}^{n} \lambda_{\beta}^{-k}\left|\dot{\mathcal{L}}_{\Delta}^{k} 1_{\beta}\right|_{1}} \leqslant \max _{1 \leqslant k \leqslant n} \frac{\left\|\dot{\mathcal{L}}_{\Delta}^{k} 1_{\beta}\right\|_{\mathcal{B}}}{\left|\dot{\mathcal{L}}_{\Delta}^{k} 1_{\beta}\right|_{1}} \leqslant M_{0} .
$$

Then since $\psi_{n}$ lies in a ball of radius $M_{0}$ in $\mathcal{B}$ for all $n$, and this ball is compact in $L^{1}(\bar{m})$, we may choose a subsequence $\left(n_{i}\right)_{i}$ such that $\psi_{n_{i}}$ converges in $L^{1}(\bar{m})$ to a function $\psi_{*} \in \mathcal{B}$ with $\left\|\psi_{*}\right\|_{\mathcal{B}} \leqslant M_{0}$. Now

$$
\begin{aligned}
\stackrel{\circ}{\mathcal{L}}_{\Delta} \psi_{*} & =\lim _{i \rightarrow \infty} \frac{\sum_{k=1}^{n_{i}} \lambda_{\beta}^{-k}{ }^{\circ} \mathcal{L}_{\Delta}^{k+1} 1_{\beta}}{\sum_{k=1}^{n_{i}} \lambda_{\beta}^{-k}\left|\dot{\mathcal{L}}_{\Delta}^{k} 1_{\beta}\right|_{1}}=\lambda_{\beta} \lim _{i \rightarrow \infty} \frac{\sum_{k=1}^{n_{i}} \lambda_{\beta}^{-k-1} \dot{\mathcal{L}}_{\Delta}^{k+1} 1_{\beta}}{\sum_{k=1}^{n_{i}} \lambda_{\beta}^{-k}\left|\dot{\mathcal{L}}_{\Delta}^{k} 1_{\beta}\right|_{1}} \\
& =\lambda_{\beta}\left[\lim _{i \rightarrow \infty} \frac{\sum_{k=1}^{n_{i}} \lambda_{\beta}^{-k} \dot{\mathcal{L}}_{\Delta}^{k} 1_{\beta}}{\sum_{k=1}^{n_{i}} \lambda_{\beta}^{-k}\left|\dot{\mathcal{L}}_{\Delta}^{k} 1_{\beta}\right|_{1}}+\frac{-\lambda_{\beta}^{-1} \stackrel{\circ}{\mathcal{L}}_{\Delta} 1_{\beta}+\lambda^{-n_{i}-1}{\stackrel{\stackrel{\mathcal{L}}{n}}{n_{i}+1}}_{\Delta} 1_{\beta}}{\sum_{k=1}^{n_{i}} \lambda_{\beta}^{-k}\left|\dot{\mathcal{L}}_{\Delta}^{k} 1_{\beta}\right|_{1}}\right] .
\end{aligned}
$$

The first fraction converges to $\psi_{*}$ by choice of the subsequence $\left(n_{i}\right)_{i}$. By the proof of Lemma 4.6 , the numerator of the second fraction has $\|\cdot\|_{\mathcal{B}}$-norm bounded above by $C n_{i}^{d-1}$, while by 4.12 , the denominator is bounded below by $C^{\prime} n_{i}^{d}$, thus the second fraction converges to 0 in $\mathcal{B}$ (and also in $\left.L^{1}(\bar{m})\right)$ as $i \rightarrow \infty$. This proves that $\stackrel{\circ}{\mathcal{L}}_{\Delta} \psi_{*}=\lambda_{\beta} \psi_{*}$, so that $\lambda_{\beta}$ is in the spectrum of $\dot{\mathcal{L}}_{\Delta}$.

Note that $\psi_{*} \geqslant 0$ and $\int \psi_{*} d \bar{m}=1$, so that necessarily $\lambda_{\beta}<1$. This is because

$$
\lambda_{\beta}^{n}=\lambda_{\beta}^{n} \int \psi_{*} d \bar{m}=\int \dot{\mathcal{L}}_{\Delta}^{n} \psi_{*} d \bar{m}=\int_{\grave{\Delta}^{n}} \psi_{*} d \bar{m} \stackrel{n \rightarrow \infty}{\longrightarrow} 0 .
$$

It follows that $\bar{\lambda} \leqslant \lambda_{\beta}<1$, which we have not assumed is true a priori.

We use the following lemma to show that in fact $\psi_{*}$ is strictly positive on all of $\stackrel{\Delta}{ }$. Define

$$
\|\psi\|_{\log }=\sup _{\ell, j} \operatorname{Lip}\left(\left.\log \psi\right|_{\AA_{\ell, j}}\right)
$$

\footnotetext{
${ }^{5}$ Here we use that for any two series of positive terms, $\frac{\sum_{k} a_{k}}{\sum_{k} b_{k}} \leqslant \sup _{k} \frac{a_{k}}{b_{k}}$, whenever $\sum_{k} b_{k}<\infty$.
} 
where $\operatorname{Lip}(\cdot)$ denotes the Lipschitz constant with respect to the metric $d_{\delta}(\cdot, \cdot)=e^{-\delta s(\cdot, \cdot)}$. For $M>0$, set $\mathcal{B}_{\log }(M)=\left\{\psi \in \mathcal{B}: \psi \geq 0,|\psi|_{1}=1,\|\psi\|_{\infty} \leqslant M,\|\psi\|_{\log } \leqslant M\right\}$.

Lemma 4.7. Let $\psi_{n}$ be defined by (4.13). There exists $M>0$ such that $\left.\psi_{n}\right|_{\Delta_{0}} \in \mathcal{B}_{\log }(M)$ for all $n \geqslant 0$.

Proof. We show the above property for the normalized transfer operator

$$
\stackrel{\mathcal{N}}{\Delta}_{\Delta}^{n} \psi:=\frac{\dot{\mathcal{L}}_{\Delta}^{n} \psi}{\left|\dot{\mathcal{L}}_{\Delta}^{n} \psi\right|_{1}},
$$

for any $\psi \in \mathcal{B}$ with $\|\psi\|_{\log }<\infty$ and $\int_{\grave{\Delta}^{n}} \psi d \bar{m}>0, \forall n$. Given such a $\psi$, clearly $\dot{\mathcal{L}}_{\Delta}^{n} \psi \geqslant 0$ and $\left|\mathcal{N}_{\Delta}^{n} \psi\right|_{1}=1$ since the normalization is well defined. So the first two properties of $\mathcal{B}_{\log }(M)$ are obviously satisfied by $\mathcal{N}_{\Delta}^{n} \psi$ for all $n \geqslant 0$.

To estimate the log-Lipschitz constant, let $x, y \in \Delta_{0}$ and denote by $u \in \AA_{\Delta}^{-n}$ and $v \in f_{\Delta}^{-n}$ two points in in the same $n$-cylinder in $\stackrel{\circ}{\Delta}$. We estimate

$$
\begin{aligned}
\stackrel{\circ}{\mathcal{L}}_{\Delta}^{n} \psi(x) & =\sum_{u \in \stackrel{\circ}{\Delta}_{\Delta}^{-n} x} \psi(u) e^{S_{n} \varphi_{\Delta}(u)} \leqslant \sum_{v \in \dot{f}_{\Delta}^{-n}(y)} e^{\|\psi\|_{\log } d_{\delta}(u, v)} \psi(v) e^{S_{n} \varphi_{\Delta}(v)}\left(1+C_{d} d_{\delta}(x, y)\right) \\
& \leqslant e^{\|\psi\|_{\log } d_{\delta}(x, y)}\left(1+C_{d} d_{\delta}(x, y)\right) \dot{\mathcal{L}}_{\Delta}^{n} \psi(y)
\end{aligned}
$$

where we have used bounded distortion (P3) in the second line and $d_{\delta}(u, v) \leqslant d_{\delta}(x, y)$ in the third. This yields

$$
\operatorname{Lip}\left(\left.\log \dot{\mathcal{L}}_{\Delta}^{n} \psi\right|_{\Delta_{0}}\right) \leqslant\|\psi\|_{\log }+C_{d}
$$

where we have used the estimate $\log (1+z) \leqslant z$ for $z \geqslant 0$. Since $\|\cdot\|_{\log }$ is scale invariant, we have $\left\|\left.\mathcal{N}_{\Delta}^{n} \psi\right|_{\Delta_{0}}\right\|_{\log } \leqslant\|\psi\|_{\log }+C_{d}$ for all $n \geqslant 0$.

Finally, we estimate the $L^{\infty}$ norm of $\left.\stackrel{\circ}{\mathcal{L}}_{\Delta}^{n} \psi\right|_{\Delta_{0}}$. Let $x \in \Delta_{0}$, and again using the notation $u \in \stackrel{\circ}{\Delta}_{\Delta}^{-n}(x)$, let $v$ denote a point in the $n$-cylinder $E_{n}(u)$ containing $u$ such that $\psi(v) \leqslant \frac{1}{\bar{m}\left(E_{n}(u)\right)} \int_{E_{n}(u)} \psi \bar{m}$. Then since $e^{S_{n} \varphi_{\Delta}(u)} \leqslant\left(1+C_{d}\right) \bar{m}\left(E_{n}(u)\right) / \bar{m}\left(\Delta_{0}, i\right)$ for some $i$ by (P3), we estimate following (4.15)

$$
\begin{aligned}
\left|\stackrel{\mathcal{L}}{\Delta}_{\Delta}^{n} \psi(x)\right| & \leqslant \sum_{u \in \stackrel{\circ}{\Delta}_{\Delta}^{-n}(x)} e^{\|\psi\|_{\log }} \psi(v)\left(1+C_{d}\right) \frac{\bar{m}\left(E_{n}(u)\right)}{\bar{m}\left(\Delta_{0, i}\right)} \\
& \leqslant C e^{\|\psi\|_{\log }} \sum_{u \in \dot{f}_{\Delta}^{-n}(x)} \int_{E_{n}(u)} \psi d \bar{m} \leqslant C e^{\|\psi\|_{\log }} \int_{\dot{\Delta}^{n}} \psi d \bar{m},
\end{aligned}
$$

where $C=\left(1+C_{d}\right) / \min _{i}\left\{\Delta_{0, i}\right\}$. Dividing by $\left|\dot{\mathcal{L}}_{\Delta}^{n} \psi\right|_{1}=\int_{\Delta^{n}} \psi d \bar{m}$ completes the estimate on the $\|\cdot\|_{\infty}$ norm.

Now since $\left\|1_{\beta}\right\|_{\log }=0$, the above argument implies $\left\|\left.\mathcal{N}_{\Delta}^{k} 1_{\beta}\right|_{\Delta_{0}}\right\|_{\infty} \leqslant C$ and $\left\|\left.\mathcal{N}_{\Delta}^{k} 1_{\beta}\right|_{\Delta_{0}}\right\|_{\log } \leqslant C_{d}$ for each $k \geqslant 1$. Equation 4.14 implies that the uniform bound on $\left\|\left.\mathcal{N}_{\Delta}^{k} 1_{\beta}\right|_{\Delta_{0}}\right\|_{\infty}$ passes to $\left\|\left.\psi_{n}\right|_{\Delta_{0}}\right\|_{\infty}$. Finally, while $\|\cdot\|_{\log }$ is not linear, it does satisfy the convex inequality,

$$
\left\|\frac{1}{n} \sum_{k=1}^{n} \lambda_{\beta}^{-k} \dot{\mathcal{L}}_{\Delta}^{k} 1_{\beta}\right\|_{\log } \leqslant \max _{1 \leqslant k \leqslant n}\left\|\dot{\mathcal{L}}_{\Delta}^{k} 1_{\beta}\right\|_{\log } \leqslant C_{d} .
$$

Finally, the scale invariance of $\|\cdot\|_{\log }$ implies that this bound passes to $\psi_{n}$ for each $n \geqslant 1$.

Since $\left\|\left.\psi_{n}\right|_{\Delta_{0}}\right\|_{\log } \leqslant M$ for all $n \geqslant 1$, we have $\left\|\left.\psi_{*}\right|_{\Delta_{0}}\right\|_{\log } \leqslant M$, and so for each $i$, either $\psi_{*}>0$ on $\Delta_{0, i}$ or $\psi_{*} \equiv 0$ on $\Delta_{0, i}$. Since for $x \in \stackrel{\circ}{\Delta} \ell_{\ell}$ by conditional invariance, $\psi_{*}(x)=\lambda_{\beta}^{-\ell} \dot{\mathcal{L}}^{\ell} \psi_{*}(x)=\lambda_{\beta}^{-\ell} \psi_{*} \circ \dot{\circ}_{\Delta}^{-\ell}(x)$, 
the second alternative implies $\psi_{*} \equiv 0$ on the entire column above $\Delta_{0, i}$. Again using conditional invariance, this implies that $\psi_{*} \equiv 0$ on every $\Delta_{0, j}$ that eventually maps to $\Delta_{0, i}$. By transitivity, this is the entire base $\Delta_{0}$ and so $\psi_{*} \equiv 0$ on $\Delta$, which is impossible since $\int \psi_{*} d \bar{m}=1$. Thus there exists $\delta_{0}>0$ such that $\psi_{*} \geqslant \delta_{0}$ on $\Delta_{0}$, and again using conditional invariance, we conclude that $\psi_{*} \geqslant \delta_{0}$ on all of $\stackrel{\circ}{\Delta}$.

The following lemma gives an equivalent expression for $\|\cdot\|_{\log }$, which will be convenient for the proof of Proposition 4.9

Lemma 4.8. Suppose $\psi>0$. For each $\Delta_{\ell, j}$,

$$
\left\|\left.\psi\right|_{\Delta_{\ell, j}}\right\|_{\log } \leqslant \sup _{x, y \in \Delta_{\ell, j}} \frac{|\psi(x)-\psi(y)|}{\psi(y) d_{\delta}(x, y)} \leqslant\|\psi\|_{\log } e^{\|\psi\|_{\log }} .
$$

Proof. Let $x, y \in \Delta_{\ell, j}, x \neq y$, and set $z=\log (\psi(x) / \psi(y))$. Then,

$$
\left|\frac{\psi(x)-\psi(y)}{\psi(y)}\right|=\left|e^{z}-1\right| \leqslant|z| e^{|z|} \leqslant\|\psi\|_{\log } e^{\|\psi\|_{\log }} d_{\delta}(x, y),
$$

proving the second inequality. The first inequality follows similarly using the fact that $\log (1+w) \leqslant$ $w$ for $w \geqslant 0$.

Proposition 4.9. $\stackrel{\circ}{\mathcal{L}}_{\Delta}$ has a spectral gap, i.e. $\lambda_{\beta}$ is simple and all other eigenvalues have modulus strictly less than $\lambda_{\beta}$.

Proof. We remark that the stronger assumptions used in [BDM] (see Remark 4.3) allow one to show that $\mathcal{N}_{\Delta}\left(\mathcal{B}_{\log }(M)\right) \subset \mathcal{B}_{\log }(M)$ and that the semi-norm $\left\|\dot{\mathcal{L}}_{\Delta}^{n} \psi\right\|_{\log }$ obeys a uniform LasotaYorke inequality for $\psi \in \mathcal{B}_{\log }(M)$. We prove neither property here under our weaker assumptions, and give a substantially different proof of the spectral gap.

We begin by assuming that there exists $g \in \mathcal{B}$ such that $\stackrel{\circ}{\mathcal{L}}_{\Delta} g=\lambda_{\beta} e^{i \omega} g$ for some $\omega \in[0,2 \pi)$. We will show this implies $\omega=0$ and $g$ is a multiple of $\psi_{*}$.

Using the fact that $g$ is an eigenfunction, we have

$$
\left.g\right|_{\check{\Delta}_{\ell}}=\left.\lambda_{\beta}^{-\ell} e^{-i \omega \ell} g\right|_{\Delta_{0} \cap f_{\Delta}^{-\ell}\left(\AA_{\ell}\right)},
$$

so that $g$ grows like $\lambda_{\beta}^{-\ell}$ times a rotation up the levels of the tower.

Since $\psi_{*} \geqslant \delta_{0}$ on $\Delta_{0}$, we may choose $K>\left.|g|_{\Delta_{0}}\right|_{\infty}$ sufficiently large so that $g_{1}:=\left(\operatorname{Re}(g)+K \psi_{*}\right) / C>$

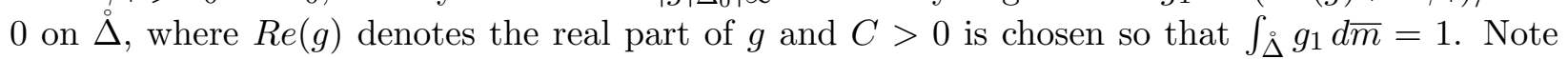
that by (4.16) and the conditional invariance of $\psi_{*}$, by choice of $K$, there exists $\delta_{1}$ such that for each $\ell \geqslant 0$,

$$
\delta_{1} \lambda_{\beta}^{-\ell} \leqslant\left. g_{1}\right|_{\Delta_{\ell}} \leqslant \delta_{1}^{-1} \lambda_{\beta}^{-\ell} .
$$

Define $g_{s}=s g_{1}+(1-s) \psi_{*}$ and $J=\left\{s \in \mathbb{R}: \inf _{\Delta} g_{s}>0\right\}$. Note that $J$ contains $[0,1]$ and by (4.17), $J$ is open. To see that in fact $J \supset \mathbb{R}^{+}$, we will use the following lemma.

Lemma 4.10. Let $\psi \in \mathcal{B}$ with $\|\psi\|_{\log }<\infty$ and let $A_{n, T} \subset \stackrel{\circ}{\Delta}^{n} \cap \stackrel{\circ}{f}_{\Delta}^{-n}\left(\Delta_{0}\right)$ be as defined in the proof of Lemma 4.5. Suppose there exists a function $r(n)$ with $r(n) \rightarrow 0$ as $n \rightarrow \infty$ such that

$$
\frac{\int_{A_{n, T}^{c}} \psi d \bar{m}}{\int_{\triangle^{n} \cap \AA_{\Delta}^{-n}\left(\Delta_{0}\right)} \psi d \bar{m}} \leqslant r(n), \quad \text { for } n \geqslant 0 .
$$

Then

$$
\left\|\left.\mathcal{N}_{\Delta}^{n} \psi\right|_{\Delta_{0}}\right\|_{\log } \leqslant C_{1} \max \left\{r(n), e^{-\delta n / T}\right\}\|\psi\|_{\log } e^{3\|\psi\|_{\log }}+C_{d}
$$

for a uniform constant $C_{1}$ depending only on $C_{d}$ and the minimum length of an element $\Delta_{0, i}$. 
Proof of Lemma. The estimate is a refined version of the one derived in the proof of Lemma 4.7 . For $x, y \in \Delta_{0}$, let $u \in f_{\Delta}^{-n}(x), v \in \dot{f}_{\Delta}^{-n}(y)$, denote corresponding points in the same $n$-cylinder. Note that for each pair of pre-images, $u \in A_{n, T}$ if and only if $v \in A_{n, T}$. In light of Lemma 4.8, it is equivalent to estimate,

$$
\begin{aligned}
& \frac{\left|\stackrel{\mathcal{L}}{\Delta}_{\Delta}^{n} \psi(x)-\dot{\mathcal{L}}_{\Delta}^{n} \psi(y)\right|}{\mathcal{L}_{\Delta}^{n} \psi(y) e^{-\delta s(x, y)}}=\frac{\sum_{u}(\psi(u)-\psi(v)) e^{S_{n} \varphi_{\Delta}(u)}}{e^{-\delta s(x, y)} \sum_{v} \psi(v) e^{S_{n} \varphi_{\Delta}(v)}}+\frac{\sum_{v} \psi(v)\left(e^{S_{n} \varphi_{\Delta}(u)}-e^{S_{n} \varphi_{\Delta}(v)}\right)}{e^{-\delta s(x, y)} \sum_{v} \psi(v) e^{S_{n} \varphi_{\Delta}(v)}} \\
& \leqslant\|\psi\|_{\log } e^{\|\psi\|_{\log }} \frac{\sum_{u} e^{-\delta s(u, v)} \psi(v) e^{S_{n} \varphi_{\Delta}(u)}}{e^{-\delta s(x, y)} \sum_{v} \psi(v) e^{S_{n} \varphi_{\Delta}(v)}}+C_{d} \\
& \leqslant\left(1+C_{d}\right)\|\psi\|_{\log } e^{\|\psi\|_{\log }}\left(e^{-\delta n / T} \frac{\sum_{v \in A_{n, T}} \psi(v) e^{S_{n} \varphi_{\Delta}(v)}}{\sum_{v} \psi(v) e^{S_{n} \varphi_{\Delta}(v)}}+\frac{\sum_{v \in A_{n, T}^{c}} \psi(v) e^{S_{n} \varphi_{\Delta}(v)}}{\sum_{v} \psi(v) e^{S_{n} \varphi_{\Delta}(v)}}\right)+C_{d} \\
& \leqslant\left(1+C_{d}\right)\|\psi\|_{\log } e^{\|\psi\|_{\log }}\left(e^{-\delta n / T}+\frac{\sum_{v \in A_{n, T}^{c}} \psi(v) e^{S_{n} \varphi_{\Delta}(v)}}{\sum_{v} \psi(v) e^{S_{n} \varphi_{\Delta}(v)}}\right)+C_{d},
\end{aligned}
$$

where we have used (P3), Lemma 4.8, and the fact that $s(u, v)-s(x, y) \geqslant n / T$ for $u, v \in A_{n, T}$. Again using bounded distortion, we may replace $e^{S_{n} \varphi_{\Delta}(v)}$ by $\bar{m}\left(E_{n}(v)\right) / \bar{m}\left(\Delta_{0, i}\right)$ for some $i \geqslant 0$, where $E_{n}(v)$ is the $n$-cylinder containing $v$; similarly,

$$
\psi(v)=e^{ \pm\|\psi\|_{\log }\left(\bar{m}\left(E_{n}(v)\right)\right)^{-1}} \int_{E_{n}(v)} \psi d \bar{m}
$$

by the log-Lipschitz continuity of $\psi$. Thus we estimate 4.18 by,

$$
\frac{\left|\dot{\mathcal{L}}_{\Delta}^{n} \psi(x)-\dot{\mathcal{L}}_{\Delta}^{n} \psi(y)\right|}{\dot{\circ}_{\Delta}^{n} \psi(y) e^{-\delta s(x, y)}} \leqslant C_{1}\|\psi\|_{\log } e^{\|\psi\|_{\log }}\left(e^{-\delta n / T}+e^{2\|\psi\|_{\log }} \frac{\int_{A_{n, T}^{c}} \psi d \bar{m}}{\int_{\check{\Delta}^{n} \cap \AA_{\Delta}^{-n}\left(\Delta_{0}\right)}^{\circ} \psi d \bar{m}}\right)+C_{d},
$$

which completes the proof of the lemma by assumption on $\psi$.

Returning to the proof of the proposition, we will apply Lemma 4.10 to $g_{s}$ for $s \in J$. Note that since $g_{s}$ is bounded away from 0 , we have $\left\|g_{s}\right\|_{\log }<\infty$. While $g_{s}$ is not an eigenfunction for $\stackrel{\circ}{\mathcal{L}}_{\Delta}$, we do have for each $n \in \mathbb{N}$,

$$
\stackrel{\circ}{\mathcal{L}}_{\Delta}^{n} g_{1}=\lambda_{\beta}^{n}\left(\operatorname{Re}\left(e^{i \omega n} g\right)+K \psi_{*}\right) / C .
$$

Thus there exists a subsequence $\left(n_{j}\right)_{j \in \mathbb{N}}$ such that $\lim _{j \rightarrow \infty} \lambda_{\beta}^{-n_{j}}{\stackrel{\circ}{\mathcal{L}} n_{j}}^{n_{1}}=g_{1}$ and it follows that

$$
\lim _{j \rightarrow \infty} \lambda_{\beta}^{-n_{j}} \stackrel{\circ}{\mathcal{L}}_{\Delta}^{n_{j}} g_{s}=g_{s} \quad \text { for each } s \in \mathbb{R} .
$$

By conformality of $\bar{m}, \int_{\Delta_{0}} \stackrel{\circ}{\mathcal{L}}_{\Delta}^{n} g_{s} d \bar{m}=\int_{\dot{\Delta}^{n} \cap \stackrel{\circ}{\Delta}_{\Delta}^{-n}\left(\Delta_{0}\right)} g_{s} d \bar{m}$ each $n \in \mathbb{N}$. On the other hand, using the definition of $g_{s}$,

$$
\int_{\Delta_{0}} \stackrel{\circ}{\mathcal{L}}_{\Delta}^{n} g_{s} d \bar{m}=\lambda_{\beta}^{n} \int_{\Delta_{0}}\left(g_{s}+s R e\left(\left(e^{i \omega n}-1\right) g\right)\right) d \bar{m} .
$$

We claim that in fact, for $s>0, s \in J$, the integral on the right must be bounded below by some $\kappa_{s}>0$, independently of $n$. If not, there is a subsequence $\left(n_{k}\right)$ such that $\int_{\Delta_{0}} \operatorname{Re}\left(\left(e^{i \omega n_{k}}-1\right) g\right) \rightarrow$ $-\frac{1}{s} \int_{\Delta_{0}} g_{s}<0$. Note that for $s^{\prime}>s$, we have

$$
\frac{g_{s}}{s}=g_{1}+\left(\frac{1}{s}-1\right) \psi_{*}>g_{1}+\left(\frac{1}{s^{\prime}}-1\right) \psi_{0}=\frac{g_{s^{\prime}}}{s^{\prime}},
$$


since $\psi_{*}>0$. So

$$
\lambda_{\beta}^{-n_{k}} \int_{\Delta_{0}}{\stackrel{\circ}{\mathcal{L}^{n}}}_{\Delta}^{n_{k}} g_{s^{\prime}}=\int_{\Delta_{0}}\left(g_{s^{\prime}}+s^{\prime} \operatorname{Re}\left(\left(e^{i \omega n_{k}}-1\right) g\right)\right) \rightarrow \int_{\Delta_{0}} s^{\prime}\left(\frac{g_{s^{\prime}}}{s^{\prime}}-\frac{g_{s}}{s}\right)<0,
$$

which implies that $g_{s^{\prime}}$ cannot be positive for any $s^{\prime}>s$, contradicting the fact that $J$ is open.

With the claim proved, we estimate, using (4.9) and the conformality of $\bar{m}$,

$$
\frac{\int_{A_{n, T}^{c}} g_{s} d \bar{m}}{\int_{\check{\Delta}^{n} \cap f_{\Delta}^{-n}\left(\Delta_{0}\right)} g_{s} d \bar{m}} \leqslant \frac{\left\|g_{s}\right\|_{\infty} \int_{A_{n, T}^{c}} 1_{\beta} d \bar{m}}{\int_{\Delta_{0}} \dot{\mathcal{L}}_{\Delta}^{n} g_{s} d \bar{m}} \leqslant \frac{\left\|g_{s}\right\|_{\infty} e^{-n(\alpha-\varepsilon)}}{\lambda_{\beta}^{n} \kappa_{s}}=: r(n),
$$

and clearly $r(n) \rightarrow 0$ at an exponential rate by choice of $\varepsilon$ and $T$.

Invoking Lemma 4.10 , since $g_{s}=\lim _{j} \lambda_{\beta}^{-n_{j}} \dot{\mathcal{L}}_{\Delta}^{n_{j}} g_{s}$, we have $\left\|\left.g_{s}\right|_{\Delta_{0}}\right\|_{\log } \leqslant C_{d}$ whenever $s \in J$. Indeed, more is true, since for each fixed $\ell>0$ and $n_{j}>\ell$,

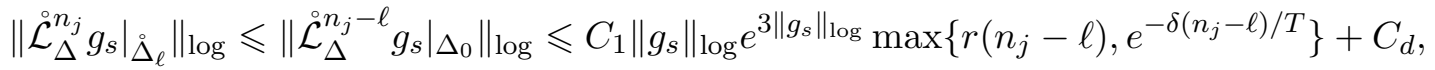

so using the scale invariance of $\|\cdot\|_{\log }$ to normalize by $\lambda_{\beta}^{-n_{j}}$ and letting $n_{j} \rightarrow \infty$, we conclude that $\left\|\left.g_{s}\right|_{\Delta_{\ell}}\right\|_{\log } \leqslant C_{d}$ for each $\ell \in \mathbb{N}$. Letting $C_{2}=\max \left\{C_{d},\left(\bar{m}\left(\Delta_{0}\right)\right)^{-1}\right\}$ and using (4.16), we have $g_{s} \in \mathcal{B}_{\log }\left(C_{2}\right)$ for each $s \in J, s>0$.

Now let $s_{0}>1$ be the right endpoint of $J$. Since $g_{s} \rightarrow g_{s_{0}}$ uniformly on each $\Delta_{\ell, j}$ as $s \rightarrow s_{0}$ from the left, we have $g_{s_{0}} \in \mathcal{B}_{\log }\left(C_{2}\right)$ as well. The following lemma from [BDM] completes the proof of the proposition.

Lemma 4.11. ([BDM, Lemma 3.2]) $g_{s_{0}}$ is bounded away from 0 on $\Delta$.

We refer the interested reader to $[\mathrm{BDM}]$ for the proof of this lemma since it requires no changes in the current setting. The proof uses only the mixing property of $\stackrel{\circ}{\Delta}_{\Delta}$ and the fact that $g_{s_{0}} \in \mathcal{B}_{\log }\left(C_{2}\right)$.

The lemma implies $s_{0} \in J$ and so $J \supset \mathbb{R}^{+}$. The fact that $g_{s}>0$ for all $s>0$ implies $g_{1} \geqslant \psi_{*}$. But since $\int g_{1} d \bar{m}=\int \psi_{*} d \bar{m}=1$, we conclude that in fact $g_{1}=\psi_{*}$. This implies that $\operatorname{Re}(g)=(C-K) \psi_{*}$ and using the linearity of $\dot{\mathcal{L}}_{\Delta}$, we conclude that $\operatorname{Im}(g)$ is also a multiple of $\psi_{*}$ and so $\omega=0$.

We have proved that $\lambda_{\beta}$ has strictly larger modulus than all other eigenvalues of $\dot{\mathcal{L}}_{\Delta}$ and that its multiplicity is one. The last step is to eliminate Jordan blocks for $\lambda_{\beta}$. Suppose there exists $g \in \mathcal{B}$ such that $\left(\stackrel{\circ}{\mathcal{L}}_{\Delta}-\lambda_{\beta} I\right) g=\psi_{*}$. It follows that $\stackrel{\circ}{\mathcal{L}}_{\Delta}^{n} g=\lambda_{\beta}^{n} g+n \lambda_{\beta}^{n-1} \psi_{*}$, so that

$$
\stackrel{\circ}{\mathcal{L}}_{\Delta}^{n}\left(g-\lambda_{\beta}^{-1} n \psi_{*}\right)=\lambda_{\beta}^{n} g .
$$

Thus for $x \in \Delta_{\ell}$,

$$
g(x)=\lambda_{\beta}^{-\ell} \dot{\mathcal{L}}_{\Delta}^{\ell}\left(g-\lambda_{\beta}^{-1} \ell \psi_{*}\right)(x)=\lambda_{\beta}^{-\ell}\left(g-\lambda_{\beta}^{-1} \ell \psi_{*}\right) \circ \stackrel{\circ}{\Delta}_{\Delta}^{-\ell}(x) .
$$

For $\ell$ sufficiently large, $g-\lambda_{\beta}^{-1} \ell \psi_{*}<0$ on $\Delta_{0}$, so there exists $L>0$ such that $g<0$ on $\cup_{\ell \geqslant L} \stackrel{\circ}{\ell}_{\ell}$. Now choose $K>0$ so large that $\bar{g}:=g-K \psi_{*}<0$ on $\cup_{\ell \leqslant L} \AA_{\ell}$. Since $\bar{g}<g$, we have $\bar{g}<0$ on $\Delta^{\circ}$. Thus for each $n$,

$$
0>\lambda_{\beta}^{-n} \int_{\dot{\Delta}^{n}} \bar{g} d \bar{m}=\lambda_{\beta}^{-n} \int_{\dot{\Delta}} \stackrel{\circ}{L}_{\Delta}^{n} \bar{g} d \bar{m}=\int_{\dot{\Delta}} \bar{g} d m+n \lambda_{\beta},
$$

which is a contradiction.

Now that we have proved the existence of a spectral gap for $\stackrel{\circ}{\mathcal{L}}_{\Delta}$, the following theorem from [BDM] follows. 
Theorem 4.12. ([BDM]) Assume $\left(f_{\Delta}, \Delta ; H\right)$ is mixing and satisfies properties (P1)-(P4). Then $\stackrel{\circ}{\mathcal{L}}_{\Delta}$ has a spectral gap. Let $\lambda$ denote the largest eigenvalue of $\stackrel{\mathcal{L}}{\Delta}_{\Delta}$ and let $\bar{g}$ denote the corresponding normalized eigenfunction.

(a) The escape rates with respect to $\bar{m}$ and $1_{\beta} \bar{m}$ exist and equal $-\log \lambda$; in particular $\lambda=\lambda_{\beta}$.

(b) $\log \lambda=\sup \left\{h_{\eta}\left(f_{\Delta}\right)+\int_{\dot{\Delta}} \varphi_{\Delta}^{H} d \eta \mid \eta \in \mathcal{M}_{f_{\Delta}}, \eta\left(-\varphi_{\Delta}^{H}\right)<\infty\right\}$.

(c) The following limit defines a probability measure $\bar{\nu}$,

$$
\bar{\nu}(\varphi)=\lim _{n \rightarrow \infty} \lambda^{-n} \int_{\AA^{n}} \psi \bar{g} d \bar{m} \quad \text { for all } \psi \in \mathcal{B}_{0}
$$

where $\mathcal{B}_{0}$ is the set of all bounded functions in $\mathcal{B}$ whose Lipschitz constant is also uniformly bounded. The measure $\bar{\nu}$ attains the supremum in (b), i.e. it is an equilibrium state for $\varphi_{\Delta}^{H}$.

(d) There exist constants $D>0$ and $\sigma_{0}<1$ such that for all $\psi \in \mathcal{B}$,

$$
\left\|\lambda^{-n} \stackrel{\circ}{\mathcal{L}}_{\Delta}^{n} \psi-d(\psi) \bar{g}\right\|_{\mathcal{B}} \leq D\|\psi\|_{\mathcal{B}} \sigma_{0}^{n}, \quad \text { where } d(\psi)=\lim _{n \rightarrow \infty} \lambda^{-n} \int_{\dot{\Delta}^{n}} \psi d \bar{m}<\infty
$$

Also, for any $\psi \in \mathcal{B}_{0}$ with $\bar{\nu}(\psi)>0$,

$$
\left|\frac{\stackrel{\circ}{\mathcal{L}}_{\Delta}^{n} \psi}{\left|\mathcal{L}_{\Delta}^{n} \psi\right|_{L^{1}(\bar{m})}}-\bar{g}\right|_{L^{1}(\bar{m})} \leqslant D\|\psi\|_{\mathcal{B}} \sigma_{0}^{n} .
$$

4.3. Application of abstract results and proof of Theorem 1.7. In this section, we complete the proof of Theorem 1.7 by projecting the results of Theorem 4.12 to our underlying map $f$ with neutral fixed point. In order to invoke these results, we fix $t \in[0,1)$ and show that our constructed tower satisfies assumptions (P1)-(P4) with respect to the measure $\bar{m}_{t}$ and potential $\varphi_{\Delta}^{H}$ induced by $t \phi^{H}-p(t)$.

(P1) follows immediately from Lemma 4.1 with $\alpha=p(t)>0$.

(P2) follows from Lemma 4.2 and the assumption that $-\log \bar{\lambda}_{t}<p(t)$.

(P4) is satisfied since $D f$ is bounded above and also below by 1 so that $S_{n} \varphi_{\Delta}(x)$ grows at most linearly in $n$ for $x \in \Delta_{0}$.

It remains to verify (P3). First choose $0<\delta \leqslant \frac{\gamma \log 2}{1+\gamma}$ for reasons to be made clear below. Standard estimates (see for example [DF, Lemma 3.1]) imply that there exists $C_{d}>0$ such that for all $n \in \mathbb{N}$ :

(D1) if $f^{n}(x) \in Y$, then $D f^{n}(x) \geqslant \max \left\{2, C_{d}^{-1} n^{1+\frac{1}{\gamma}}\right\}$;

(D2) if $f^{i}(x), f^{i}(y)$ lie in the same element of $\mathcal{P}_{1}$ for each $0 \leqslant i \leqslant n$, then

$$
\left|\log \frac{D f^{n}(x)}{D f^{n}(y)}\right| \leqslant C_{d}\left|f^{n}(x)-f^{n}(y)\right|^{\frac{\gamma}{\gamma+1}}
$$

We will need the following lemma.

Lemma 4.13. Suppose $\delta / \log 2 \leqslant q \leqslant 1$. Let $\psi \in C^{q}(I)$ and define $\tilde{\psi}$ on $\Delta$ by $\tilde{\psi}=\psi \circ \pi$. Then $|\tilde{\psi}|_{\infty} \leqslant|\psi|_{\infty}$ and $\operatorname{Lip}(\tilde{\psi}) \leqslant C|\psi|_{C^{q}}$ for some constant $C$ depending on the minimum length of $\Delta_{0, i}$. 
Proof. The bound $|\tilde{\psi}|_{\infty} \leqslant|\psi|_{\infty}$ is immediate. To prove the bound on the Lipschitz norm of $\tilde{\psi}$, let $x, y \in \Delta_{\ell, j}$ and estimate

$$
\frac{|\tilde{\psi}(x)-\tilde{\psi}(y)|}{d_{\delta}(x, y)}=\frac{|\psi(\pi(x))-\psi(\pi(y))|}{|\pi(x)-\pi(y)|^{q}} \cdot \frac{|\pi(x)-\pi(y)|^{q}}{e^{-\delta s(x, y)}} .
$$

The first ratio above is bounded by $|\psi|_{C^{q}(I)}$. To estimate the second ratio, note that if $s(x, y)=n$, then by construction of $\Delta, f^{i}(\pi(x))$ and $f^{i}(\pi(y))$ lie in the same element of $\mathcal{P}_{1}$ for all $i \leqslant \tau^{n}(x)$. By (D1), $D f^{\tau^{n}}(\pi(x)) \geqslant 2^{n}$, so that using also (D2), $|\pi(x)-\pi(y)| \leqslant C 2^{-n}$. Thus $|\pi(x)-\pi(y)|^{q} \leqslant C e^{-\delta n}$ as long as $q \geqslant \delta / \log 2$, as required.

By (D2) above, we see that the potential $\phi=-\log D f$ is Hölder continuous with exponent $\gamma /(1+\gamma)$. By Lemma 4.13, the induced potential $\varphi_{\Delta}$ will be Lipschitz in the metric $d_{\delta}$ if $\frac{\delta}{\log 2} \leqslant \frac{\gamma}{1+\gamma}$, i.e. if $\delta \leqslant \frac{\gamma \log 2}{1+\gamma}$, which is what we chose initially. Then (D2) also implies the bounded requirement of (P3) for $e^{S_{n} \phi}$ with this choice of $\delta$.

This extends to the potential $t S_{n} \phi-n p(t)$ for $t \in[0,1]$ since

$$
\begin{aligned}
\mid e^{t S_{n} \phi(x)-n p(t)} & -e^{t S_{n} \phi(y)-n p(t)}\left|=e^{t S_{n} \phi(y)-n p(t)}\right| e^{t S_{n} \phi(x)-t S_{n} \phi(y)}-1 \mid \\
& \leqslant C t\left|\log e^{S_{n} \phi(x)-S_{n} \phi(y)}\right| \leqslant C^{\prime} t\left|f^{n}(x)-f^{n}(y)\right|^{\frac{\gamma}{1+\gamma}},
\end{aligned}
$$

where we have used the fact that $\phi \leqslant 0$ and $p(t) \geqslant 0$. Thus (P3) is satisfied with this choice of $\delta$ for all $t \in[0,1]$.

The final point to check is that $f_{\Delta}$ is transitive and aperiodic on the partition $\left\{\Delta_{\ell, j}\right\}$. This is implied by the following.

Lemma 4.14. Suppose that $H$ is non-swallowing. Then for each base $\Delta_{0, i}$, there exists $N=N(i)$ such that $f^{n}\left(\Delta_{0, i}\right) \supseteq \Delta_{0}$ for all $n \geqslant N$.

Proof. Transitivity of $\stackrel{\circ}{f}_{\Delta}$ is obvious by the non-swallowing assumption (1) on $\mathcal{Q}$ and $\Delta_{0}=\cup_{Q \in \mathcal{Q}} Q$. It remains to verify aperiodicity.

By property (2) of the definition of non-swallowing, there exists $i_{0} \in \mathbb{N}$ such that $\Delta_{0, i_{0}} \supset(1 / 2,1 / 2+$ $\delta$ ) for some $\delta>0$. Let $\Delta_{0, j} \subseteq \stackrel{\circ}{F}\left(\Delta_{0, i_{0}}\right)$. Since $\Delta_{0, i_{0}}$ is recurrent, there exists $n_{0} \in \mathbb{N}$ such that $f^{n_{0}}\left(\Delta_{0}, j\right) \supseteq \Delta_{0, i_{0}}$. Now due to the renewal structure and the fact that $\Delta_{0, i_{0}}$ contains all $Y_{k}$ for $k$ greater than some $k_{0}$, if $f^{n_{1}}\left(\Delta_{0, i_{0}}\right) \supseteq \Delta_{0, j}$, then also $f^{n_{1}+1}\left(\Delta_{0, i_{0}}\right) \supseteq \Delta_{0, j}$. So we have both $f^{n_{0}+n_{1}}\left(\Delta_{0, i_{0}}\right) \supseteq \Delta_{0, i_{0}}$ and $f^{n_{0}+n_{1}+1}\left(\Delta_{0, i_{0}}\right) \supseteq \Delta_{0, i_{0}}$. Thus $f_{\Delta}$ is aperiodic.

With properties $(\mathrm{P} 1)-(\mathrm{P} 4)$ verified, we conclude by the results of the previous section that $\dot{\mathcal{L}}_{\Delta}$ has a spectral gap on $\mathcal{B}$ and the conclusions of Theorem 4.12 apply.

The last step in the proof of Theorem 1.7 is to show that we can project the results of Theorem 4.12 to our open system $\left(\stackrel{\circ}{f}, m_{t} ; H\right)$. For this we need the following proposition, which is an adaption of [BDM, Prop. 4.2].

Proposition 4.15. Recall the projection $P_{\pi, t}$ defined by 4.2 with respect to the measure $m_{t}$. Let $C^{q}(\stackrel{\circ}{I})$ be the set of Hölder continuous functions with exponent q supported on $\stackrel{\circ}{I}$. Then $\operatorname{Lip}\left(\left.J_{t} \pi\right|_{\Delta_{\ell}}\right)<$ $\infty$ for each $\ell$ and $C^{q}(\stackrel{i}{I}) \subset P_{\pi, t} \mathcal{B}_{0}$ for all $q \geqslant \delta / \log 2$. 
Proof. Due to conformality and the definition of $\bar{m}_{t}$, for $x \in \Delta_{\ell}, x=f_{\Delta}^{\ell}(y)$, we have

$$
J_{t} \pi(x)=\frac{d m_{t}(\pi x)}{d \bar{m}_{t}(x)}=\frac{d m_{t}\left(\pi\left(f_{\Delta}^{\ell} y\right)\right)}{d \bar{m}_{t}(y)}=\frac{d m_{t}\left(f^{\ell}(\pi y)\right)}{d m_{t}(\pi y)}=e^{-t S_{\ell} \phi(\pi y)+\ell p(t)} .
$$

Now by the proof of Lemma 4.13 , $J_{t} \pi$ is Lipschitz in the metric $d_{\delta}$ with Lipschitz constant depending only on the level $\ell$ and the distortion constant from (D2).

Now let $\psi \in C^{q}(\stackrel{\circ}{I})$ for some $q \geqslant \delta / \log 2$. Recall from the proof of Lemma 4.2 the index set $\mathcal{K}$ of pairs $(\ell, j)$ such that $\ell \leqslant L, \pi\left(\cup_{(\ell, j) \in \mathcal{K}} \Delta_{\ell, j}\right)=\pi(\stackrel{\circ}{\Delta})$, and $\pi\left(\Delta_{\ell, j}\right) \cap \pi\left(\Delta_{\ell^{\prime}, j^{\prime}}\right)=\emptyset$ for all pairs $(\ell, j) \neq\left(\ell^{\prime}, j^{\prime}\right)$ in $\mathcal{K}$.

For $(\ell, j) \in \mathcal{K}$ and $x \in \Delta_{\ell, j}$, define $\tilde{\psi}(x)=\psi \circ \pi(x) J_{t} \pi(x)$. Define $\tilde{\psi} \equiv 0$ elsewhere on $\Delta$. Then it follows from fact that $J_{t} \pi$ is Lipschitz on $\cup_{\ell \leqslant L} \Delta_{\ell}$ and Lemma 4.13 that $\tilde{\psi} \in \mathcal{B}_{0}$. Also, since the images $\pi\left(\Delta_{\ell, j}\right)$ and $\pi\left(\Delta_{\ell^{\prime}, j^{\prime}}\right)$ are disjoint for all pairs $(\ell, j) \neq\left(\ell^{\prime}, j^{\prime}\right)$ in $\mathcal{K}$, we have $P_{\pi, t} \tilde{\psi}=\psi$.

We proceed to prove the items of Theorem 1.7. Fix $q>0$ as in the statement of Theorem 1.7 and choose $\delta>0$, such that $\delta \leqslant \min \left\{\frac{q}{\log 2}, \frac{\gamma \log 2}{1+\gamma}\right\}$ so that the conditions of Lemma 4.13 and Proposition 4.15 are satisfied as well as (P3).

(1) The characterization of $\lambda_{t}<1$ as the spectral radius and the existence of a spectral gap for $\dot{\mathcal{L}}_{\Delta}$ follow immediately from Lemma 4.1 and Theorem 4.12 .

It follows from the definitions of $\stackrel{\circ}{\mathcal{L}}_{t \phi^{H}-p(t)}$ and $\stackrel{\circ}{\mathcal{L}}_{\varphi_{\Delta}^{H}}$ that given $\psi \in C^{q}(\pi(\stackrel{\circ}{\Delta}))$, and $\tilde{\psi}$ as defined above in the proof of Proposition 4.15, that

$$
\stackrel{\circ}{\mathcal{L}}_{t \phi^{H}-p(t)}^{n} \psi=P_{\pi, t} \stackrel{\circ}{\mathcal{L}}_{\varphi_{\Delta}^{H}}^{n} \tilde{\psi}, \quad \forall n \geqslant 0 .
$$

This relation also holds if $\psi$ is supported on $H \cup \pi(\stackrel{\Delta}{)})$ since the part of $\psi$ on $H$ is deleted in one step.

Define $g_{t}=P_{\pi, t} \bar{g}_{t}$. Since $\bar{g}_{t}$ is bounded away from 0 on $\stackrel{\triangleright}{\Delta}$, it follows using the index set $\mathcal{K}$ from the proof of Lemma 4.2 that $g_{t}$ is bounded away from 0 on $\stackrel{\circ}{I}$. Moreover, by 4.19 , we have $\stackrel{\circ}{\mathcal{L}}_{t} g_{t}=P_{\pi, t}{\stackrel{\circ}{\varphi_{\varphi}^{H}}} \bar{g}_{t}=\lambda_{t} g_{t}$.

(2), (3) and (4). Define $\nu_{t}=\pi_{*} \bar{\nu}_{t}$. Now since

$$
\int_{\tilde{I}} \psi d m_{t}=\int_{\dot{\Delta}} \tilde{\psi} d \bar{m}_{t}
$$

the characterization of $\nu_{t}(\psi)$ as the limit of $\lambda_{t}^{-n} \int_{I^{n}} \psi g_{t} d m_{t}$ follows from 44.19) and Theorem 4.12(c).

With this definition, $\nu_{t}$ satisfies

$$
\log \lambda_{t}=h_{\nu_{t}}(f)+\int t \phi^{H} d \nu_{t}-p(t)
$$

due to Theorem 4.12(b) and (c). To project this relation from $\Delta$ to $I$, we use Lemma 4.1, the definition of $\varphi^{H}$ and the fact that $\pi: \Delta \rightarrow I$ is at most countable-to-one to deduce $h_{\nu_{t}}(f)=h_{\bar{\nu}_{t}}\left(f_{\Delta}\right)$.

Indeed, $\nu_{t}$ attains the supremum of pressures over all measures $\eta$ that lift to $\Delta$. However, due to Proposition 1.3, we conclude that the supremum of measures that lift to $\Delta$ is in fact the supremum of all ergodic, invariant measures supported on $\stackrel{\circ}{I}^{\infty}$. Thus $\log \lambda_{t}=p^{H}(t)-p(t)$, completing the proof of item (2). Moreover, it follows immediately that $\nu_{t}$ is the equilibrium state for the potential $t \phi^{H}-p^{H}(t)$. 
To complete the proof of items (3) and (4), we need only construct the conformal measure $m_{t}^{H}$. To this end, define,

$$
m_{t}^{H}(\psi)=\lim _{n \rightarrow \infty} \lambda_{t}^{-n} \int_{I^{n}} \psi d m_{t} .
$$

The limit exists again using 4.19 and Theorem $4.12(\mathrm{~d})$. It follows that $m_{t}^{H}$ is supported on $i^{\infty}$, and indeed that $\nu_{t}=g_{t} m_{t}^{H}$, completing item (4).

To see that $m_{t}^{H}$ is conformal, note that for a small interval $A \subset I$ centered at a $m_{t}^{H}$-typical point $x$, we have

$$
\begin{aligned}
\frac{m_{t}^{H}(A)}{m_{t}^{H}(f(A))} & =\lim _{n \rightarrow \infty} \frac{\lambda_{t}^{-n-1} \int_{I^{n+1}} 1_{A} d m_{t}}{\lambda_{t}^{-n} \int_{I^{n}} 1_{f(A)} d m_{t}}=\lim _{n \rightarrow \infty} \frac{\lambda_{t}^{-n-1} \int_{I^{n}}{\stackrel{\circ}{\mathcal{L}_{t \phi^{H}}-p(t)}}\left(1_{A}\right) d m_{t}}{\lambda_{t}^{-n} \int_{I^{n}} 1_{f(A)} d m_{t}} \\
& =\lim _{n \rightarrow \infty} \frac{\lambda_{t}^{-1} \int_{I^{n}} 1_{f(A)} \cdot e^{t \phi^{H} \circ f_{A}^{-1}-p(t)} d m_{t}}{\int_{I^{n}} 1_{f(A)} d m_{t}}
\end{aligned}
$$

where $f_{A}=\left.f\right|_{A}$ is injective on $A$. Taking the limit as $A \rightarrow\{x\}$, we have

$$
\frac{m_{t}^{H}(x)}{m_{t}^{H}(f(x))}=\lambda_{t}^{-1} e^{t \phi^{H}(x)-p(t)}=e^{t \phi^{H}(x)-p^{H}(t)},
$$

using item (2), which proves item (3).

(5) The characterization of $\mu_{t}^{H}:=g_{t} m_{t}$ as a conditionally invariant measure and a limiting distribution follows immediately from Theorem 4.12(d), again using 4.19 in addition to Proposition 4.15 , which allows us to lift any $\psi \in C^{q}(\stackrel{\circ}{I})$ to the function space $\mathcal{B}_{0}$ on $\Delta$. The convergence extends also to $\psi$ supported fully on $I$ since in one iterate, $\dot{\mathcal{L}}_{t \phi^{H}-p(t)} \psi$ is supported on $\stackrel{\circ}{I}$ so the definition of $\psi$ on $I \backslash \stackrel{\circ}{I}$ is irrelevant to the value of the limit.

4.4. Proof of Corollary 1.8. First note that the statement $p^{H}(t)>0$ if and only if $t<t^{H}$ is simply the definition of $t^{H}$ together with Proposition 1.6(a).

We next prove $-\log \bar{\lambda}_{t}<p(t)$ if and only if $p^{H}(t)>0$. Recall that by Proposition 1.3 , we have

$$
\log \bar{\lambda}_{t} \geqslant \log \underline{\lambda}_{t} \geqslant p^{H}(t)-p(t)
$$

Assume $p^{H}(t)>0$. Then by the above inequality, $\log \bar{\lambda}_{t}>-p(t)$ and we are in the setting of Theorem 1.7: the associated transfer operator on the tower has a spectral gap and in particular, the escape rate exists, $\lambda_{t}=\bar{\lambda}_{t}=\underline{\lambda}_{t}$ and the variational principle holds,

$$
\log \lambda_{t}=p^{H}(t)-p(t) .
$$

On the other hand, assume $\log \bar{\lambda}_{t}>-p(t)$. Then again we are in the setting of Theorem 1.7 and the same set of results holds, including the variational principle. This implies in particular, that $p^{H}(t)=p(t)+\log \lambda_{t}>0$.

Finally, we show that $\lambda_{t}$ exists and the variational principle holds in all cases. The only case that remains to be addressed is when $p^{H}(t)=0$. In this case, Proposition 1.3 implies

$$
\log \bar{\lambda}_{t} \geqslant \log \underline{\lambda}_{t} \geqslant-p(t)
$$

In fact, all inequalities must be equalities otherwise $\log \bar{\lambda}_{t}>-p(t)$ and again we are in the case of the spectral gap which forces $p^{H}(t)>0$ by Theorem 1.7 , contrary to our assumption. 
4.5. Proof of Theorem 1.9. Let $z \in I$ and let $H_{\varepsilon} \supset\{z\}, \varepsilon \in\left(0, \varepsilon_{0}\right)$ be a nested sequence of intervals that are nonswallowing holes. Our first step is to show that the sequence of conditionally invariant densities $g_{t}^{H_{\varepsilon}}$ given by item (2) of Theorem 1.7 enjoy some uniform regularity in $\varepsilon$. To this end we define the variation of a function $\psi$ on an interval $J=[c, d]$ by,

$$
\bigvee_{J} \psi:=\sup \sum_{i=1}^{n}\left|\psi\left(x_{i}\right)-\psi\left(x_{i-1}\right)\right|
$$

where the supremum is taken over all finite collections of points $\left\{x_{i}\right\}_{i=0}^{n}$ such that $c=x_{0}<x_{1}<$ $\cdots<x_{n}=d$. $\psi$ is said to be of bounded variation on $J$ if $\bigvee_{J} \psi<\infty$. We call the set of such functions $B V(J)$.

Before we state our estimate on the variation of our densities, observe that since $g_{t}^{H_{\varepsilon}}=P_{\pi, t} \bar{g}_{t}^{H_{\varepsilon}}$, the fact that $\inf _{\Delta} \bar{g}_{t}^{H_{\varepsilon}}>0$ and the existence of the set of partition elements $\mathcal{K}$ from Lemma 4.2 imply that

$$
\inf _{I \backslash H_{\varepsilon}} g_{t}^{H_{\varepsilon}}=: c_{\varepsilon}>0
$$

Lemma 4.16. There exists $\varepsilon_{1}>0$ and a constant $C_{t}>0$ such that $\bigvee_{I} g_{t}^{H_{\varepsilon}} \leqslant C_{t}$ for all $\varepsilon \in\left[0, \varepsilon_{1}\right]$

Proof. In what follows, for brevity, we denote $\stackrel{\circ}{\mathcal{L}}_{t \phi^{H_{\varepsilon}}-p(t)}$ by $\stackrel{\circ}{\mathcal{L}}_{t}$. Let $\left\{L_{j, \varepsilon}^{n}\right\}_{j}$ denote the images under $f^{n}$ of the finitely many intervals $\left\{K_{j, \varepsilon}^{n}=\left(a_{j}^{n}, b_{j}^{n}\right)\right\}_{j}$ of monotonicity for $f^{n}$. Let $\xi_{j}^{n}$ denote the inverse branch of $f^{n}$ on $L_{j, \varepsilon}^{n}$. Since $e^{t S_{n} \phi-n p(t)} \leqslant e^{-n p(t)}$, by standard estimates (see also the proof Lemma 6.4), we have for $\psi \in B V(I)$ and $n \geqslant 0$,

$$
\begin{aligned}
\bigvee_{I} \dot{\mathcal{L}}_{t}^{n} \psi & \leqslant \sum_{j} \bigvee_{L_{j, \varepsilon}^{n}}\left(\psi e^{t S_{n} \phi-n p(t)}\right) \circ \xi_{j}^{n}+\sum_{j}\left|\psi\left(a_{j}^{n}\right)\right| e^{t S_{n} \phi\left(a_{j}^{n}\right)-n p(t)}+\left|\psi\left(b_{j}^{n}\right)\right| e^{t S_{n} \phi\left(b_{j}^{n}\right)-n p(t)} \\
& \leqslant \sum_{j} e^{-n p(t)} \bigvee_{K_{j, \varepsilon}^{n}} \psi+\sup _{K_{j, \varepsilon}^{n}}|\psi| \bigvee_{K_{j, \varepsilon}^{n}} e^{t S_{n} \phi-n p(t)}+e^{-n p(t)} \sum_{j}\left(\bigvee_{K_{j, \varepsilon}^{n}} \psi+2 \inf _{K_{j, \varepsilon}^{n}}|\psi|\right) \\
& \leqslant 3 e^{-n p(t)} \bigvee_{I} \psi+\frac{C_{d}}{\min _{j} m_{t}\left(K_{j, \varepsilon}^{n}\right)} \int_{I_{\varepsilon}^{n}}|\psi| d m_{t},
\end{aligned}
$$

where $C_{d}$ is a distortion constant depending only on $t$ and $f$ and $\stackrel{\circ}{I}_{\varepsilon}^{n}=\cap_{i=0}^{n} f^{-i}\left(I \backslash H_{\varepsilon}\right)$. Also, we used the fact that $e^{t S_{n} \phi-n p(t)}$ is monotonic on each interval to bound

$$
\bigvee_{K_{j, \varepsilon}^{n}} e^{t S_{n} \phi-n p(t)} \leqslant \sup _{K_{j, \varepsilon}^{n}} e^{t S_{n} \phi-n p(t)} \leqslant e^{-n p(t)} .
$$

Now using Corollary 1.8 and letting $\lambda_{\varepsilon}$ denote the largest eigenvalue of $\stackrel{\circ}{\mathcal{L}}_{t}$, we estimate,

$$
\bigvee_{I} \lambda_{\varepsilon}^{-n} \dot{\mathcal{L}}_{t}^{n} \psi \leqslant 3 e^{-n p^{H_{\varepsilon}}(t)} \bigvee_{I} \psi+\frac{C_{d}}{\min _{j} m_{t}\left(K_{j, \varepsilon}^{n}\right)} \lambda_{\varepsilon}^{-n} \int_{\dot{I}_{\varepsilon}^{n}}|\psi| d m_{t} .
$$

Since $t<t^{H_{\varepsilon}}$, we have $p^{H_{\varepsilon}}(t)>0$ so we may choose $n$ sufficiently large that $3 e^{-n p^{H_{\varepsilon}}(t)}=: \rho_{\varepsilon}<1$. Then iterating the above relation, we estimate for all $k \geqslant 0$,

$$
\bigvee_{I} \lambda_{\varepsilon}^{-k n} \stackrel{\circ}{\mathcal{L}} k n_{t}^{k n} \leqslant \rho_{\varepsilon}^{k} \bigvee_{I} \psi+\frac{C_{d}}{\min _{j} m_{t}\left(K_{j, \varepsilon}^{n}\right)} \sum_{j=1}^{k} \rho_{\varepsilon}^{j} \lambda_{\varepsilon}^{-(k-j) n} \int_{\dot{I}_{\varepsilon}^{(k-j) n}}|\psi| d m_{t} .
$$


Since $\lambda_{\varepsilon}^{-k n}{ }_{\mathcal{L}_{t}^{k n}}^{k n} \rightarrow c_{1} g_{t}^{H_{\varepsilon}}$ as $k \rightarrow \infty$ for some $c_{1}>0$ by Theorem 1.7 , we will apply 4.22 to $\psi \equiv 1$ to conclude that $g_{t}^{H_{\varepsilon}} \in B V(I)$. First note that by (4.21),

$$
\lambda_{\varepsilon}^{-k} \int_{I_{\varepsilon}^{k}} 1 d m_{t} \leqslant c_{\varepsilon}^{-1} \lambda_{\varepsilon}^{-k} \int_{I_{\varepsilon}^{k}} g_{t}^{H_{\varepsilon}} d m_{t}=c_{\varepsilon}^{-1}
$$

for all $k \geqslant 0$ using the conditional invariance of $g_{t}^{H_{\varepsilon}}$, i.e. $\int_{I_{\varepsilon}} g_{t}^{H_{\varepsilon}} d m_{t}=\lambda_{\varepsilon}^{k}$. Using this together with 4.22 yields,

$$
\bigvee_{I} \lambda_{\varepsilon}^{-k n}{\stackrel{\circ}{\mathcal{L}_{t}} k n}^{k n} \leqslant \frac{C_{d}}{\min _{j} m_{t}\left(K_{j, \varepsilon}^{n}\right)} \frac{c_{\varepsilon}^{-1}}{1-\rho_{\varepsilon}} .
$$

Thus $\bigvee_{I} g_{t}^{H_{\varepsilon}} \leqslant \frac{C_{d}}{c_{1} c_{\varepsilon}\left(1-\rho_{\varepsilon}\right) \min _{j} m_{t}\left(K_{j, \varepsilon}^{n}\right)}$, so that $g_{t}^{H_{\varepsilon}} \in B V(I)$.

Once we know $g_{t}^{H_{\varepsilon}} \in B V(I)$, we may apply 4.22 once more with $\psi=g_{t}^{H_{\varepsilon}}$ to obtain,

$$
\bigvee_{I} g_{t}^{H_{\varepsilon}}=\bigvee_{I} \lambda_{\varepsilon}^{-k n} \dot{\mathcal{L}}_{t}^{k n} g_{t}^{H_{\varepsilon}} \leqslant \rho_{\varepsilon}^{k} \bigvee_{I} g_{t}^{H_{\varepsilon}}+\frac{C_{d}}{\min _{j} m_{t}\left(K_{j, \varepsilon}^{n}\right)} \sum_{j=1}^{k} \rho_{\varepsilon}^{j},
$$

and letting $k \rightarrow \infty$, we conclude

$$
\bigvee_{I} g_{t}^{H_{\varepsilon}} \leqslant \frac{C_{d}}{\left(1-\rho_{\varepsilon}\right) \min _{j} m_{t}\left(K_{j, \varepsilon}^{n}\right)} .
$$

Finally, we may choose this constant to be independent of $\varepsilon$ for $\varepsilon$ sufficiently small. This is because $\rho_{\varepsilon} \leqslant \rho_{\varepsilon_{0}}<1$ by monotonicity of $p^{H_{\varepsilon}}(t)$ in $\varepsilon$. Also, since the sequence of holes is nested by assumption and $f^{n}$ has finitely many branches, $\min _{j} m_{t}\left(K_{j, \varepsilon}^{n}\right)$ can only increase for $\varepsilon$ sufficiently small.

Now consider the sequence of measures $\mu_{t}^{H_{\varepsilon}}=g_{t}^{H_{\varepsilon}} m_{t}$ for $\varepsilon>0$. Define the BV norm, $\left\|g_{t}^{H_{\varepsilon}}\right\|_{B V}=$ $\bigvee_{I} g_{t}^{H_{\varepsilon}}+\left|g_{t}^{H_{\varepsilon}}\right|_{L^{1}\left(m_{t}\right)}$. Since $\left\|g_{t}^{H_{\varepsilon}}\right\|_{B V} \leqslant C_{t}+1$ for all $\varepsilon<\varepsilon_{1}$, and BV is compact in $L^{1}\left(m_{t}\right)$, any limit point of the sequence must be absolutely continuous with respect to $m_{t}$ and in fact, have a density in $B V(I)$.

Fix a subsequence $\left\{\varepsilon_{n}\right\}_{n \in \mathbb{N}}$ such that $\left\{g_{t}^{H_{\varepsilon_{n}}}\right\}_{n \in \mathbb{N}}$ converges in $L^{1}\left(m_{t}\right)$ to a density $h_{t} \in B V(I)$ with $\left\|h_{t}\right\|_{B V} \leqslant C_{t}+1$. Let $\mu_{\infty}=h_{t} m_{t}$.

We claim $\mu_{\infty}$ must be invariant as well, making it the unique invariant measure $\mu_{t}$ for $f$ absolutely continuous with respect to $m_{t}$. To see this, recall the following characterization of the spectral radius,

$$
\lambda_{t}^{H_{\varepsilon}}=\int_{\stackrel{\circ}{\mathcal{L}}} \stackrel{\circ}{t}_{t}\left(g_{t}^{H_{\varepsilon}}\right) d m_{t}=\int_{\check{I}^{1}} g_{t}^{H_{\varepsilon}} d m_{t}=1-\int_{\check{I} \backslash \AA^{1}} g_{t}^{H_{\varepsilon}} d m_{t} .
$$

Due to the uniform integrability of $g_{t}^{H_{\varepsilon}}$ given by the proof of Lemma 4.16 , it follows that $\lambda_{t}^{H_{\varepsilon}} \rightarrow 1$ as $\varepsilon \rightarrow 0$.

Now,

$\left|\mathcal{L}_{t} h_{t}-h_{t}\right|_{1} \leqslant\left|\mathcal{L}_{t} h_{t}-\lambda_{\varepsilon_{n}}^{-1} \stackrel{\circ}{\mathcal{L}}_{t} g_{t}^{H_{\varepsilon_{n}}}\right|_{1}+\left|g_{t}^{H_{\varepsilon_{n}}}-h_{t}\right|_{1} \leqslant\left|1-\lambda_{\varepsilon_{n}}^{-1}\right|\left|\mathcal{L}_{t} h_{t}\right|_{1}+\lambda_{\varepsilon_{n}}^{-1}\left|\mathcal{L}_{t} h_{t}-\stackrel{\circ}{\mathcal{L}}_{t} g_{t}^{H_{\varepsilon_{n}}}\right|_{1}+\left|g_{t}^{H_{\varepsilon_{n}}}-h_{t}\right|_{1}$

The first and third terms above clearly approach 0 as $n \rightarrow \infty$. We split the second term again,

$$
\left|\mathcal{L}_{t} h_{t}-\stackrel{\circ}{\mathcal{L}}_{t} g_{t}^{H_{\varepsilon_{n}}}\right|_{1} \leqslant\left|\mathcal{L}_{t} h_{t}-\stackrel{\circ}{\mathcal{L}}_{t} h_{t}\right|_{1}+\left|\stackrel{\mathcal{L}}{t}_{t}\left(h_{t}-g_{t}^{H_{\varepsilon_{n}}}\right)\right|_{1} \leqslant \int_{I \backslash \stackrel{I}{I}_{\varepsilon_{n}}^{1}} h_{t} d m_{t}+\left|h_{t}-g_{t}^{H_{\varepsilon_{n}}}\right|_{1},
$$

and again both terms vanish as $n \rightarrow \infty$, using the fact that $|h|_{\infty} \leqslant C_{t}+1$. 


\section{Proof of Theorem 1.11: The Case Where the exponential tail equals the EXPONENTIAL ESCAPE: $t \in\left[t^{H}, 1\right)$}

Although Theorem 1.11 applies to all $t \in[0,1)$, it provides new information only for $t \in\left[t^{H}, 1\right)$. For ease of notation, we will denote $\stackrel{\circ}{\mathcal{L}}_{t}=\dot{\mathcal{L}}_{t \phi^{H}-p(t)}$ in this section. We do not prove that $\dot{\mathcal{L}}_{t}$ has a spectral gap for $t \in\left[t^{H}, 1\right)$, yet we will show that all limits points of the sequence $\left\{\frac{\dot{\mathcal{L}}_{t}^{n} 1}{\left|\dot{\mathcal{L}}_{t}^{n} 1\right|_{L^{1}\left(m_{t}\right)}}\right\}_{n \in \mathbb{N}}$ are absolutely continuous with respect to $m_{t}$. We will also use an averaging technique to construct absolutely continuous conditionally invariant probability measures with eigenvalue $\lambda_{t}$.

Let $g_{t}^{0}$ denote the invariant probability density for the transfer operator before the introduction of the hole, $\mathcal{L}_{t}$. By Lemma 4.16 applied to $\varepsilon=0, g_{t}^{0} \in B V(I)$ and 4.21) implies that

$$
\frac{\inf _{I} g_{t}^{0}}{\sup _{I} g_{t}^{0}}=: c_{1}>0 \text {. }
$$

Moreover, since each $x \in \stackrel{\circ}{I}$ has at least one preimage under $f$ by definition of $H$ being nonswallowing, we have

$$
\inf _{x \in I} \stackrel{\circ}{\mathcal{L}}_{t} 1(x) \geqslant e^{-p(t)} \inf _{x \in I}|D f(x)|^{-t}=: c_{0}>0
$$

First we prove the following lemma.

Lemma 5.1. There exists $C, \eta>0$ such that for all $n \in \mathbb{N},\left|\dot{\mathcal{L}}_{t}^{n} 1\right|_{\infty} \leqslant C(1-\eta)^{n}$.

Proof. Since $H$ is a union of $N_{0}$-cylinders by assumption, it follows that $\#\left\{y \in f^{-N_{0}}(x) \cap H\right\} \geqslant 1$ for each $x \in I$. Thus

$$
\dot{\mathcal{L}}_{t}^{N_{0}} g_{t}^{0}(x)=\mathcal{L}_{t}^{N_{0}} g_{t}^{0}(x)-\mathcal{L}_{t}^{N_{0}}\left(1_{I \backslash I^{N_{0}}} g_{t}^{0}\right)(x) \leqslant g_{t}^{0}(x)\left[1-c_{1} \mathcal{L}_{t}^{N_{0}}\left(1_{I \backslash I^{N_{0}}}\right)(x)\right] \leqslant g_{t}^{0}(x)\left[1-c_{1} c_{0}^{N_{0}}\right] .
$$

Iterating this relation, we obtain $\dot{\mathcal{L}}_{t}^{k N_{0}} g_{t}^{0}(x) \leqslant\left(1-c_{1} c_{0}^{N_{0}}\right)^{k} g_{t}^{0}(x)$, for each $k \geqslant 0$ and $x \in \stackrel{\circ}{I}$. Using again the upper and lower bounds on $g_{t}^{0}$, we complete the proof of the lemma with $1-\eta=$ $\left(1-c_{1} c_{0}^{N_{0}}\right)^{1 / N_{0}}$.

Next we address the regularity of $\mathcal{N}_{t}^{n} 1:=\frac{\dot{\mathcal{L}}_{t}^{n} 1}{\left|\dot{\mathcal{L}}_{t}^{n} 1\right|_{1}}$ to show that all limit points of the sequence are absolutely continuous with respect to $m_{t}$. Here, $|\cdot|_{1}$ denotes the $L^{1}\left(m_{t}\right)$-norm.

To this end, for $q>0$, define the log-Hölder constant (which might be $\infty$ ) of a function $\psi \geqslant 0$ by

$$
\|\psi\|_{q, \log }=\sup _{J \in \mathcal{P}_{N_{0}}} \sup _{x, y \in J}|x-y|^{-q}|\log \psi(x) / \psi(y)| .
$$

If $\psi \equiv 0$ on an element of $\mathcal{P}_{N_{0}}$, we simply set its Hölder constant on that element equal to 0 .

The following lemma is essentially [DF, Prop. 3.7], adapted to the potentials $t \phi^{H}-p(t)$. It is also of note that the constants appearing below are uniform in $H$ and $N_{0}$.

Lemma 5.2. Fix $t$ and let $H$ be an element of $\mathcal{P}_{N_{0}}$. For all $n \in \mathbb{N}$,

$$
\left\|\mathcal{N}_{t}^{n} 1\right\|_{q, \log } \leqslant t C_{d}
$$

where $C_{d}$ is from property (D2) and $q=\gamma /(1+\gamma)$. 
Proof. Fix $n \in \mathbb{N}, J \in \mathcal{P}_{N_{0}}$ and for $x, y \in J$, denote by $x_{i}$ (resp. $y_{i}$ ) the pre-images $f^{-n}(x)$ (resp. $\left.f^{-n}(y)\right)$ such that each pair $x_{i}, y_{i}$ lies in the same branch of $\dot{f}^{-n}$. Now,

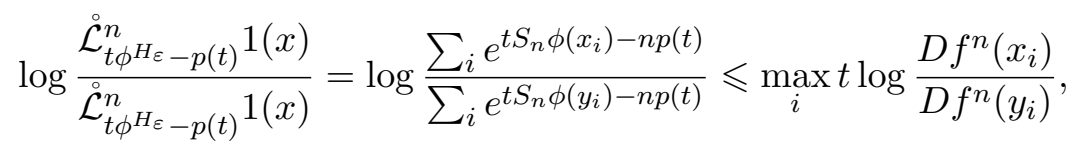

where we have used footnote 5. The last quantity above is bounded by $t C_{d}|x-y|^{\gamma /(1+\gamma)}$ by the standard distortion estimate (D2) introduced in the verification of (P3) in the proof of Theorem 1.7. proving the lemma.

By Lemma 5.2, we have a uniform bound on $\left\|\mathcal{N}_{t}^{n} 1\right\|_{q, \log }, n \in \mathbb{N}$. By $[\mathrm{DF}$, Lemma 3.6] any sequence of functions with a uniform bound on the log-Hölder constant lies in a compact set in the space of probability measures on $I$ and any (weak) limit point $\mu$ must be of the form,

$$
\mu=s \delta_{0}+(1-s) \mu_{*}, \quad \text { for some } s \in[0,1],
$$

where $\delta_{0}$ is the point mass at 0 and $d \mu_{*}=\psi_{*} d m_{t}$ for some function $\psi_{*}$ with $\left\|\psi_{*}\right\|_{q, \log } \leqslant t C_{d}$.

Now suppose $\mu$ is the limit of $\mathcal{N}_{t}^{n_{j}} 1$, for some subsequence $\left(n_{j}\right)_{j \in \mathbb{N}}$ such that $s>0$. It follows that for each $k \in \mathbb{N}$,

$$
\lim _{j \rightarrow \infty} \frac{\dot{\mathcal{L}}_{t}^{n_{j}+k} 1}{\left|\dot{\mathcal{L}}_{t}^{n_{j}} 1\right|_{1}}=\dot{\mathcal{L}}^{k} \mu=s \delta_{0}+(1-s) \stackrel{\circ}{\mathcal{L}}^{k} \mu_{*}
$$

Thus for each $k \in \mathbb{N}$, we can find $n_{j}$ large enough that

$$
\frac{\left|\mathcal{L}_{t}^{n_{j}+k} 1\right|_{1}}{\left|\mathcal{L}_{t}^{n_{j}} 1\right|_{1}} \geqslant \frac{s}{2}
$$

But choosing $k$ sufficiently large so that $C(1-\eta)^{k}<\frac{s}{3}$, we have by Lemma 5.1 for all $n_{j} \geqslant 0$,

$$
\frac{\left|\dot{\mathcal{L}}_{t}^{n_{j}+k} 1\right|_{1}}{\left|\mathcal{L}_{t}^{n_{j}} 1\right|_{1}}=\frac{\int_{I^{n_{1}}}{\stackrel{\circ}{\mathcal{L}_{t}^{k}}}^{k} 1 d m_{t}}{\int_{\stackrel{I}{ }^{n_{j}}} 1 d m_{t}} \leqslant \sup _{\dot{I}} \mathcal{\circ}_{t}^{k} 1 \leqslant C(1-\eta)^{k}<\frac{s}{3},
$$

which is a contradiction. Thus for any limit point $\mu$, we must have $s=0$. This proves the first part of Theorem 1.11.

We next address conditionally invariant measures obtained as averages of $\mathcal{L}_{t}^{n} 1$, suitably renormalized. Unfortunately, the naive average $\frac{1}{n} \sum_{i=0}^{n-1} \frac{\dot{\mathcal{L}}_{t}^{i} 1}{\left|\dot{\mathcal{L}}_{t}^{i} 1\right|_{1}}$ does not yield a conditionally invariant measure in general since the operation $\psi \mapsto \frac{\stackrel{\bullet}{\mathcal{L}}_{t} \psi}{\left|\mathcal{L}_{t} \psi\right|_{1}}$ is not linear. Thus we adopt the point of view taken in [CMM] (see also [DY]).

Define $b_{j}=j^{t\left(1+\frac{1}{\gamma}\right)-1}, Z_{n}=\sum_{j=1}^{n} \lambda_{t}^{-j} b_{j}\left|\mathcal{L}_{t}^{j} 1\right|_{1}$ and

$$
\psi_{n}=\frac{1}{Z_{n}} \sum_{i=1}^{n} \lambda_{t}^{-j} b_{j} \stackrel{\mathcal{L}}{j}_{t}^{j} 1
$$

By Lemma 5.2 and using the convexity of $\|\cdot\|_{q, \log }, \psi_{n}$ is a sequence of probability densities with $\left\|\psi_{n}\right\|_{q, \log } \leqslant t C_{d}$ for each $n$. Thus by [DF, Lemma 3.6], any limit point of this sequence must again be of the form (5.3). 
Suppose $\mu$ is the limit of $\left\{\psi_{n_{j}}\right\}_{j \in \mathbb{N}}$. Then since $\mu$ gives 0 weight to the discontinuities of $\stackrel{\circ}{\mathcal{L}}_{t} 1$, we have

$$
\begin{aligned}
& \stackrel{\circ}{\mathcal{L}}_{t} \mu=\lim _{j \rightarrow \infty} \frac{1}{Z_{n_{j}}} \sum_{i=1}^{n_{j}} \lambda_{t}^{-i} b_{i} \stackrel{\mathcal{L}}{\mathcal{L}}_{t}^{i+1} 1 \\
& =\lim _{j \rightarrow \infty}\left[\frac{\lambda_{t}}{Z_{n_{j}}} \sum_{i=1}^{n_{j}} \lambda_{t}^{-i} b_{i} \stackrel{\circ}{\mathcal{L}}_{t}^{i} 1+\frac{\lambda_{t}}{Z_{n_{j}}} \sum_{i=1}^{n_{j}} \lambda_{t}^{-i} b_{i} \stackrel{\circ}{\mathcal{L}}_{t}^{i} 1\left(\frac{b_{i-1}}{b_{i}}-1\right)-\frac{b_{0} \stackrel{\circ}{\mathcal{L}}_{t} 1}{Z_{n_{j}}}+\frac{\lambda_{t}^{-n_{j}} b_{n_{j}} \dot{\mathcal{L}}_{t}^{n_{j}+1} 1}{Z_{n_{j}}}\right],
\end{aligned}
$$

where $b_{0}:=b_{1}=1$. The first term on the right hand side clearly converges to $\lambda_{t} \mu$, while the second term converges to 0 (in $L^{1}\left(m_{t}\right)$ ) since $\lim _{i \rightarrow \infty} \frac{b_{i-1}}{b_{i}}=1$.

Next, consider the normalization factors $Z_{n}$. By the conformality of $m_{t}$, we have

$$
\int \dot{\mathcal{L}}_{t}^{n} 1 d m_{t}=\int_{I^{n}} 1 d m_{t} \geqslant \bigcup_{k \geqslant n} m_{t}\left(J_{k}\right) \geqslant \sum_{k \geqslant n+h} C k^{-t\left(1+\frac{1}{\gamma}\right)} e^{-k p(t)} \geqslant C(n+h)^{-t\left(1+\frac{1}{\gamma}\right)+1} e^{-n p(t)} .
$$

Since $\lambda_{t}=e^{-p(t)}$, it follows that

$$
\lambda_{t}^{-i} b_{i}\left|\dot{\mathcal{L}}_{t}^{i} 1\right|_{1} \geqslant C^{\prime} \quad \text { for all } i \geqslant 1,
$$

which implies that the sequence $Z_{n}$ is increasing and unbounded. Thus the third term on the right hand side of (5.4) converges to 0 (again in $L^{1}\left(m_{t}\right)$ ) as $j \rightarrow \infty$. Finally, the fourth term converges to 0 as well, since the numerator is the final summand of the subexponentially diverging series in the denominator.

We have shown that $\stackrel{\circ}{\mathcal{L}}_{t} \mu=\lambda_{t} \mu$ and iterating this relation yields $\stackrel{\circ}{\mathcal{L}}_{t}^{n} \mu=\lambda_{t}^{n} \mu$, which implies $s=0$ so that $\mu=\psi_{*} m_{t}$ is an absolutely continuous conditionally invariant probability measure with eigenvalue $\lambda_{t}$. Indeed, due to the regularity of $\psi_{n}$, which is inherited by $\psi_{*}, \psi_{n}$ converges pointwise uniformly to $\psi_{*}$ on each element of $\mathcal{P}_{N_{0}}$. Thus the convergence of $\psi_{n}$ to $\psi_{*}$ holds in $L^{1}\left(m_{t}\right)$.

\section{Proof of Theorem 1.13}

In this section, we will work principally with the domain $Y:=[1 / 2,1]$ and the induced map $F=f^{\tau}: Y \circlearrowleft$, where $\tau$ is the first return time to $Y$ defined earlier. Recall that $F$ has countably many branches created by the preimages of the intervals $J_{n}, n \geqslant 0$. Let $Y_{n} \subset[1 / 2,1]$ be the interval such that $f\left(Y_{n}\right)=J_{n}$. Then $F\left(Y_{n}\right)=Y$ for each $n \geqslant 0$ so that $F$ is a full-branched Gibbs-Markov map.

Now fix a family of holes $\left(H_{\varepsilon}\right)_{\varepsilon \leqslant \varepsilon_{0}}$ satisfying assumption $\mathbf{( H )}$ of Section 1.4 . Unlike in previous sections, the holes $H_{\varepsilon}$ are not required to be elements of a Markov partition for $f$.

Since we are interested in the limit as $H_{\varepsilon}$ shrinks to a point, and thus $t^{H_{\varepsilon}}$ increases to 1 , we will make a standing assumption throughout this section that

$$
t^{H_{\varepsilon}}>\frac{2 \gamma}{1+\gamma}
$$

Our goal is to construct an invariant measure on the survivor set compatible with the punctured potential $t \phi^{H_{\varepsilon}}-p^{H_{\varepsilon}}(t)$ and then show that this sequence of singular measures converges weakly to the absolutely continuous (with respect to $m_{t}$ ) equilibrium state $\mu_{t}$ for the closed system as $\varepsilon \rightarrow 0$.

This program will be carried out in several steps. We first derive a uniform bound on the essential spectral radius of the transfer operator associated with the induced punctured potential $t \Phi^{H_{\varepsilon}}-$ $\tau p^{H_{\varepsilon}}(t)$ (Section 6.1) and then show that for small holes it has a spectral gap (Section 6.2). In 
Section 6.3 we use the spectral gap to construct an invariant measure for $\stackrel{\circ}{F}$ supported on $Y$. This measure projects to an invariant measure for $\stackrel{\circ}{f}$ on $\stackrel{\circ}{I}^{\infty}$ and items (1)-(5) of Theorem 1.13 are proved in Sections 6.4 and 6.5 .

Since we normalize the induced potential $t \Phi^{H_{\varepsilon}}$ by the punctured pressure $p^{H_{\varepsilon}}(t)$ rather than $p(t)$, the measure $m_{t}$ is no longer a conformal measure for this potential. In order to proceed, we first prove the existence of a conformal measure for this potential.

Recall the definition of the variation of a function $\psi$ on an interval $J$ defined by $(4.20$ ).

Lemma 6.1. For $t<1$, let

$$
P_{t, \varepsilon}=P\left(t \Phi-\tau p^{H_{\varepsilon}}(t)\right) .
$$

Then $P_{t, \varepsilon}>0$ and there exists a $\left(t \Phi-\tau p^{H_{\varepsilon}}(t)-P_{t, \varepsilon}\right)$-conformal measure $\tilde{m}_{t, H_{\varepsilon}}$ for $F$ on $Y$, which has no atoms.

Proof. First note that $P(t \Phi-\tau p(t))=0$. So using the fact that $\tau \geqslant 1$, we have

$$
0=P\left(t \Phi-\tau p(t)+\tau p^{H_{\varepsilon}}(t)-\tau p^{\varepsilon} H(t)\right) \leqslant p^{H_{\varepsilon}}(t)-p(t)+P\left(t \Phi-\tau p^{H_{\varepsilon}}(t)\right) .
$$

If $H_{\varepsilon}$ is a Markov hole, then since $p^{H_{\varepsilon}}(t)-p(t)=\log \lambda_{t}<0$ by Corollary 1.8, we conclude that $P_{t, \varepsilon}>0$ as required. On the other hand, if $H_{\varepsilon}$ is not a Markov hole, we can always find $H_{\varepsilon^{\prime}} \subset H_{\varepsilon}$ such that $H_{\varepsilon^{\prime}}$ is a Markov hole. Then the above argument implies $P_{t, \varepsilon^{\prime}}>0$ so that by monotonicity, $P_{t, \varepsilon}>0$ as well.

In order to prove the existence of $\tilde{m}_{t, H_{\varepsilon}}$, we will check that the potential $t \Phi-\tau p^{H_{\varepsilon}}(t)$ is contracting in the sense of [LSV1].

(i) $e^{t \Phi-\tau p^{H_{\varepsilon}}(t)}$ is of bounded variation. Note that for each $n, \tau$ is constant on $Y_{n}$, while $\left.e^{t \Phi}\right|_{Y_{n}}=e^{t S_{n} \phi}$ is monotonically decreasing. Thus the variation of $e^{t \Phi-\tau p^{H_{\varepsilon}}(t)}$ is bounded by

$$
\bigvee_{Y} e^{t \Phi-\tau p^{H_{\varepsilon}}(t)} \leqslant \sum_{n=0}^{\infty} \sup _{Y_{n}} e^{t \Phi-\tau p^{H_{\varepsilon}}(t)} \leqslant \sum_{n=0}^{\infty} C(n+1)^{t\left(1+\frac{1}{\gamma}\right)} e^{-p^{H_{\varepsilon}}(t)(n+1)}
$$

and the series converges for all $t \in[0,1]$ since for $t<t^{H_{\varepsilon}}$, we have $p^{H_{\varepsilon}}(t)>0$, while for $t \geqslant t^{H_{\varepsilon}}$, we have $t\left(1+\frac{1}{\gamma}\right)>1$ by our standing assumption 6.1) that $t^{H_{\varepsilon}}>\frac{2 \gamma}{1+\gamma}$. Thus $e^{t \Phi-\tau p^{H_{\varepsilon}}(t)}$ has bounded variation.

(ii) $\sum_{n \geqslant 0} \sup _{Y_{n}} e^{t \Phi-\tau p^{H_{\varepsilon}}(t)}<\infty$. This is the same calculation as above.

(iii) There exists $n_{0} \in \mathbb{N}$ such that

$$
\sup _{Y} e^{t S_{n_{0}} \Phi-\tau^{n_{0}} p^{H_{\varepsilon}}(t)}<\inf _{Y} \mathcal{L}_{t \Phi-\tau p^{H_{\varepsilon}}(t)}^{n_{0}} 1 .
$$

This is trivial since the left hand side decreases exponentially in $n_{0}$, while the right hand side is greater than $\inf _{Y} \mathcal{L}_{t \Phi-\tau p(t)}^{n_{0}} 1$. Since $F$ is full-branched Gibbs Markov and the spectral radius of $\mathcal{L}_{t \Phi-\tau p(t)}$ on $C^{1}(Y)$ is $1, \mathcal{L}_{t \Phi-\tau p(t)}^{n_{0}} 1$ converges uniformly on $Y$ to a smooth invariant density for $F$ which is bounded below away from 0 .

Now that we have verified that the potential $t \Phi-\tau p^{H_{\varepsilon}}(t)$ is contracting, we may apply [LSV1, Theorem 3.1] to conclude the existence of the $\left(t \Phi-\tau p^{H_{\varepsilon}}(t)-P_{t, \varepsilon}\right)$-conformal measure $\tilde{m}_{t, H_{\varepsilon}}$.

The importance of $\tilde{m}_{t, H_{\varepsilon}}$ is that it enables us to compute the escape of mass from $Y$ under $\stackrel{\circ}{F}$ via a change of variables. Define $\Psi_{1, \varepsilon}=t \Phi-\tau p^{H_{\varepsilon}}(t)-P_{t, \varepsilon}$ and the corresponding punctured potential by $\Psi_{1, \varepsilon}^{H}=t \Phi^{H_{\varepsilon}}-\tau p^{H_{\varepsilon}}(t)-P_{t, \varepsilon}$. Let $\tilde{H}_{\varepsilon}$ be the hole in $Y$ induced by $H_{\varepsilon}$ and let $\stackrel{\circ}{\varepsilon}_{\varepsilon}^{n}=\cap_{i=0}^{n} F^{-i}\left(Y \backslash \tilde{H}_{\varepsilon}\right)$ 
denote the set of points in $Y$ which do not escape in the first $n$ iterates of the induced map $F$. The corresponding transfer operators are denoted

$$
\stackrel{\circ}{\mathcal{L}}_{\Psi_{1, \varepsilon}^{H}} \psi=\mathcal{L}_{\Psi_{1, \varepsilon}}\left(1_{Y_{\varepsilon}^{\prime}} \psi\right)
$$

Thus,

$$
\int_{Y} \dot{\mathcal{L}}_{\Psi_{1, \varepsilon}^{n}}^{n} \psi d \tilde{m}_{t, H_{\varepsilon}}=\int_{\dot{\Upsilon}^{n}} \psi d \tilde{m}_{t, H_{\varepsilon}},
$$

so that the rate of escape with respect to $\tilde{m}_{t, H_{\varepsilon}}$ is governed by the spectral radius of ${\stackrel{\circ}{\mathcal{L}^{H}}}_{1, \varepsilon}$. We note that for $t=1$, the existence of such a conformal measure is trivial since $p^{H_{\varepsilon}}(1)=p(1)=0$ and $m_{1}$ remains the conformal measure for $\mathcal{L}_{\Phi}$.

6.1. Lasota-Yorke Inequalities for the Induced Potentials. In the next two sections, we will establish strong spectral properties for the induced open system $\stackrel{\circ}{F}_{\varepsilon}: \stackrel{\circ}{Y}_{\varepsilon}^{1} \rightarrow Y$ and transfer operators with respect to several potentials. Recall $\Psi_{1, \varepsilon}$ and $\Psi_{1, \varepsilon}^{H}$ defined above. Similarly, define $\Psi_{2}=t \Phi-\tau p(t)$ and its punctured counterpart $\Psi_{2}^{H_{\varepsilon}}=t \Phi^{H_{\varepsilon}}-\tau p(t)$. We will study the spectral properties of the corresponding transfer operators on spaces of functions of bounded variation.

For $\psi \in B V(Y)$, define $\|\psi\|_{B V}=\bigvee_{Y} \psi+|\psi|_{L^{1}\left(m_{t}\right)}$. In this section, we prove the following proposition.

Proposition 6.2. Let $\left\{H_{\varepsilon}\right\}_{\varepsilon \leqslant \varepsilon_{0}}$ be a nested family of intervals satisfying assumption $(\mathbf{H})$. There exist constants $C>0$ and $\sigma_{1}<1$ such that for all $\psi \in B V(Y), \varepsilon \in\left[0, \varepsilon_{0}\right]$ and $n \geqslant 0$,

$$
\left\|\dot{\mathcal{L}}_{\Psi_{2}^{H_{\varepsilon}}}^{n} \psi\right\|_{B V} \leqslant C \sigma_{1}^{n}\|\psi\|_{B V}+C|\psi|_{L^{1}\left(m_{t}\right)} .
$$

As a consequence, the essential spectral radius of ${\stackrel{\mathcal{L}}{\Psi_{2}^{H_{\varepsilon}}}}$ as an operator on $B V(Y)$ is uniformly bounded by $\sigma_{1}$ for all $\varepsilon \in\left[0, \varepsilon_{0}\right]$.

Remark 6.3. Similarly, if we define the norm $\|\psi\|_{B V, H_{\varepsilon}}=\bigvee_{Y} \psi+|\psi|_{L^{1}\left(\tilde{m}_{t, H_{\varepsilon}}\right)}$, one can also prove the inequality

$$
\left\|{\stackrel{\mathcal{L}}{\Psi_{1, \varepsilon}^{H}}}^{n} \psi\right\|_{B V, H_{\varepsilon}} \leqslant C \sigma_{1}^{n}\|\psi\|_{B V, H_{\varepsilon}}+C|\psi|_{L^{1}\left(\tilde{m}_{t, H_{\varepsilon}}\right)}
$$

following closely the proof of Proposition 6.2 , and showing directly that the essential spectral radius

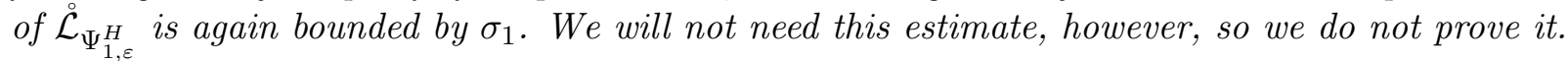

Proposition 6.2 together with Lemma 6.7 suffice to prove quasi-compactness of $\stackrel{\circ}{\mathcal{L}}_{\Psi_{2}^{H_{\varepsilon}}}$ for all $\varepsilon>0$ sufficiently small.

Before proceeding to the proof of Proposition 6.2, we make some observations about our holes $H_{\varepsilon}$. Let $\tilde{H}_{\varepsilon}$ be the hole in $Y$ induced by $H_{\varepsilon}$ and recall the sets $\left\{Y_{i, j}\right\}_{i, j \geqslant 0}$ defined in Section 1.4 . which denote the maximal intervals on which $\tau^{2}$ is constant, $Y_{i, j}=Y_{i} \cap F^{-1}\left(Y_{j}\right)$. Since $F$ is full branched and $H_{\varepsilon}$ has finitely many components, it follows that $\stackrel{\circ}{F}^{2}:=\left.F^{2}\right|_{\dot{Y}_{\varepsilon}^{2}}$ enjoys the finite images condition: The set $\left\{\stackrel{\circ}{F}^{2}\left(Y_{i, j}\right)\right\}_{i, j \geqslant 0}$ comprises a finite union of intervals. The number of these intervals varies depending on the placement of $H_{\varepsilon}$, but is uniformly bounded above.

For example, suppose $z=H_{0} \subset[0,1 / 2]$ lies in the interior of one of the intervals $J_{i_{0}, j_{0}}:=J_{i_{0}} \cap$ $f_{L}^{-n}\left(Y_{j_{0}}\right)$, for some $i_{0} \geqslant 1, j_{0} \geqslant 0$, where $f_{L}$ is the left branch of $L$. It follows from condition $(\mathbf{H})$ that $H_{\varepsilon} \subset J_{i_{0}, j_{0}}$ for $\varepsilon \leqslant \varepsilon_{0}$ since otherwise, as $\varepsilon \rightarrow 0$, image intervals of arbitrarily short length would be created. For $i \in \mathbb{N}$ and $j<i_{0}, j \neq j_{0}$, we have $\stackrel{\circ}{\varepsilon}_{\varepsilon}^{2}\left(Y_{i, j}\right)=Y$, while for $j \geqslant i_{0}, j \neq j_{0}$, we have $\stackrel{\circ}{F}_{\varepsilon}^{2}\left(Y_{i, j}\right)=Y \backslash f^{i_{0}}\left(H_{\varepsilon}\right)$, which is a union of two intervals, $A_{1}=\left[1 / 2, a_{1}\right]$ and $A_{2}=\left[a_{2}, 1\right]$. Note that $f^{i_{0}}(H) \subset Y_{j_{0}}$. It remains to consider the intervals $Y_{i, j}$ with $j=j_{0}$. If $i<i_{0}$ and $j_{0}<i_{0}$, 
then again, $\stackrel{\circ}{F}_{\varepsilon}^{2}\left(Y_{i, j_{0}}\right)=Y$, while if $j_{0} \geqslant i_{0}$ then $\stackrel{\circ}{F}_{\varepsilon}^{2}\left(Y_{i, j_{0}}\right)=Y \backslash f^{i_{0}}\left(H_{\varepsilon}\right)$. If $i \geqslant i_{0}$ and $j<i_{0}$, then $\stackrel{\circ}{F}_{\varepsilon}^{2}\left(Y_{i, j_{0}}\right)=Y \backslash F\left(f^{i_{0}} H_{\varepsilon}\right)$, again the union of two intervals, $A_{3}=\left[1 / 2, a_{3}\right]$ and $A_{4}=\left[a_{4}, 1\right]$. Finally, if $i \geqslant i_{0}$ and $j_{0} \geqslant i_{0}$, then $\stackrel{\circ}{F}_{\varepsilon}^{2}\left(Y_{i, j_{0}}\right)=Y \backslash\left(f^{i_{0}}\left(H_{\varepsilon}\right) \cup F\left(f^{i_{0}} H_{\varepsilon}\right)\right)$, which can be at most 3 intervals, $A_{5}=\left[1 / 2, a_{5}\right], A_{6}=\left[a_{6}, 1\right]$ and $A_{7}=\left[a_{7}, a_{8}\right]$. Other cases for $z$ on the boundary of two consecutive $J_{i, j}$ or in $[1 / 2,1]$ are similar. In all cases, our assumption (H) guarantees that the minimum length of these image intervals is uniformly bounded away from 0 in $\varepsilon$, making it possible to obtain uniform Lasota-Yorke inequalities.

Finally, although $1_{Y \backslash \tilde{H}_{\varepsilon}}$ has infinite variation when $H_{\varepsilon} \subset[0,1 / 2], \mathcal{L}_{\Psi_{1, \varepsilon}}\left(1_{Y \backslash \tilde{H}_{\varepsilon}}\right)$ has finite variation since $\mathcal{L}_{\Psi_{1, \varepsilon}}\left(1_{Y \backslash \tilde{H}_{\varepsilon}}\right)$ is smooth on each of the finitely many images of $Y$ under $\stackrel{\circ}{F}$. The same holds true for $1_{Y \backslash\left(\tilde{H}_{\varepsilon} \cup F^{-1}\left(\tilde{H}_{\varepsilon}\right)\right)}$ and ${\stackrel{\mathcal{L}}{\Psi_{1, \varepsilon}}}^{2}\left(1_{Y \backslash\left(\tilde{H}_{\varepsilon} \cup F^{-1}\left(\tilde{H}_{\varepsilon}\right)\right)}\right)$ as well as $\stackrel{\circ}{\mathcal{L}}_{\Psi_{2}}^{2}\left(1_{Y \backslash\left(\tilde{H}_{\varepsilon} \cup F^{-1}\left(\tilde{H}_{\varepsilon}\right)\right)}\right)$

Lemma 6.4. Let $\left(H_{\varepsilon}\right)_{\varepsilon \leqslant \varepsilon_{0}}$ be a family of holes satisfying assumption $(H)$. Then there exist constants $C_{3}>0$ and $\sigma<1$, independent of $\varepsilon \leqslant \varepsilon_{0}$, such that for all $\psi \in B V(Y)$,

$$
\left\|{\stackrel{\mathcal{L}}{\Psi_{2}^{H \varepsilon}}}^{H_{\varepsilon}} \psi\right\|_{B V} \leqslant \sigma\|\psi\|_{B V}+C_{3}|\psi|_{L^{1}\left(m_{t}\right)} .
$$

Proof. Despite the countably many components of $\tilde{H}_{\varepsilon}$, the proof follows the standard line. We include it to show that there is sufficient contraction uniformly for $t \in[0,1]$ and that the constants are independent of $\varepsilon$ under assumption $(\mathbf{H})$.

For convenience, let us reindex the countably many intervals on which $\stackrel{\circ}{F}^{2}$ is smooth and injective by $Z_{n}=\left[a_{n}, b_{n}\right], n \in \mathbb{N}$. Note that each $Z_{n} \subset Y_{i, j}$ for some pair $(i, j)$, although some $Y_{i, j}$ will contain two or at most three $Z_{n}$ as described earlier. We denote by $\xi_{n}$ the inverse of $\stackrel{\circ}{F}^{2}$ restricted to $Z_{n}$. For brevity, we will denote the potential for $\stackrel{\circ}{\mathcal{L}}_{\Psi_{2}^{H_{\varepsilon}}}^{2}$ by $G=\sum_{i=0}^{1}\left(t \Phi^{H_{\varepsilon}}-\tau p(t)\right) \circ \stackrel{\circ}{F}_{\varepsilon}^{i}$. Then for $\psi \in B V(Y)$, we write,

$$
\begin{aligned}
\bigvee_{Y} \dot{\mathcal{L}}_{\Psi_{2}^{H_{\varepsilon}}}^{2} \psi=\bigvee_{Y} & \left(\sum_{n} \psi \circ \xi_{n} \cdot e^{G \circ \xi_{n}}\right) \leqslant \sum_{n} \bigvee_{F^{2}\left(Z_{n}\right)}\left(\psi \circ \xi_{n} \cdot e^{G \circ \xi_{n}}\right) \\
& +\sum_{n}|\psi| \circ \xi_{n}\left(a_{n}\right) \cdot e^{G \circ \xi_{n}\left(a_{n}\right)}+|\psi| \circ \xi_{n}\left(b_{n}\right) \cdot e^{G \circ \xi_{n}\left(b_{n}\right)},
\end{aligned}
$$

Note that the sum over the endpoints $a_{n}=1 / 2$ or $b_{n}=1$ may be omitted so that most intervals (excepting those of type $A_{7}$ described above) will have at most one endpoint to consider, and the full branched ones none at all. Since we must estimate the worst case, however, we will not keep track of these differences in our estimates.

For an interval $J$ on which $\xi_{n}$ is smooth, we estimate

$$
\begin{aligned}
\bigvee_{J} \psi \circ \xi_{n} \cdot e^{G \circ \xi_{n}} & =\bigvee_{\xi_{n}(J)} \psi \cdot e^{G} \leqslant \sup _{\xi_{n}(J)} e^{G} \bigvee_{\xi_{n}(J)} \psi+\sup _{\xi_{n}(J)}|\psi| \bigvee_{\xi_{n}(J)} e^{G} \\
& \leqslant \sup _{\xi_{n}(J)} e^{G}\left(2 \bigvee_{\xi_{n}(J)} \psi+\frac{1}{m_{t}\left(\xi_{n}(J)\right)} \int_{\xi_{n}(J)}|\psi| d m_{t}\right)
\end{aligned}
$$

where we have estimated $\bigvee_{\xi_{n}(J)} e^{G} \leqslant \sup _{\xi_{n}(J)} e^{G}$ since $G$ is monotonic on each $\xi_{n}(J)$.

Let $\sigma_{n}<1$ denote the maximum of $e^{G}$ restricted to $Z_{n}$. Using bounded distortion and the conformality of $m_{t}$, we have

$$
\sup _{\xi_{n}(J)} e^{G} \frac{1}{m_{t}\left(\xi_{n}(J)\right)} \leqslant \frac{C_{d}}{m_{t}(J)} .
$$


Combining this with our previous estimates, we bound the variation of $\psi \circ \xi_{n} \cdot e^{G \circ \xi_{n}}$ using (6.3),

$$
\bigvee_{J} \psi \circ \xi_{n} \cdot e^{G \circ \xi_{n}} \leqslant 2 \sigma_{n} \bigvee_{\xi_{n}(J)} \psi+\frac{C_{d}}{m_{t}(J)} \int_{\xi_{n}(J)}|\psi| d m_{t}
$$

It remains to estimate the sum over endpoints in 6.2 . Now

$$
\begin{aligned}
|\psi| \circ \xi_{n}\left(a_{n}\right) \cdot e^{G \circ \xi_{n}\left(a_{n}\right)} & +|\psi| \circ \xi_{n}\left(b_{n}\right) \cdot e^{G \circ \xi_{n}\left(b_{n}\right)} \leqslant \sigma_{n}\left(|\psi| \circ \xi_{n}\left(a_{n}\right)+|\psi| \circ \xi_{n}\left(b_{n}\right)\right) \\
& \leqslant \sigma_{n}\left(\inf _{Z_{n}} \psi+\bigvee_{Z_{n}} \psi\right) \leqslant \sigma_{n}\left(\frac{1}{m_{t}\left(Z_{n}\right)} \int_{Z_{n}} \psi d m_{t}+\bigvee_{Z_{n}} \psi\right) \\
& \leqslant C_{d} m_{t}\left(\stackrel{\circ}{F}_{\varepsilon}^{2}\left(Z_{n}\right)\right)^{-1} \int_{Z_{n}} \psi d m_{t}+\sigma_{n} \bigvee_{Z_{n}} \psi,
\end{aligned}
$$

where we have used the bounded distortion estimate 6.4 in the last step.

Using these estimates together with 6.5 in 6.2 yields,

$$
\bigvee_{Y} \dot{\mathcal{L}}_{\Psi_{2}^{H \varepsilon}}^{2} \psi \leqslant \sum_{n} 3 \sigma_{n} \bigvee_{Z_{n}} \psi+2 C_{d} \sup _{n}\left\{m_{t}\left(\stackrel{\circ}{F}_{\varepsilon}^{2}\left(Z_{n}\right)\right)^{-1}\right\} \int_{Y}|\psi| d m_{t}
$$

Sublemma 6.5. There exists $\sigma<1$ such that for all $t \in[0,1]$ and all $\gamma \in(0,1), \max _{n} 3 \sigma_{n} \leqslant \sigma$.

Sublemma 6.5 completes the proof of Lemma 6.4 using the estimate above, the fact that $\sum_{n} \bigvee_{Z_{n}} \psi \leqslant$ $\bigvee_{Y} \psi$ and assumption $(\mathbf{H})$ that the lengths (and therefore the $m_{t}$-measures) of the image intervals are bounded below away from 0 by a constant independent of $\varepsilon$ and $t$.

Proof of Sublemma 6.5. We want to maximize $e^{G}$ on $Y$ and show that this maximum is less than $1 / 3$. For $t=1$, this maximum is $1 / 4$ since $p^{H}(1)=p(1)=0$. From now on, we assume $t<1$.

We begin by estimating the weakest contraction due to

$$
e^{t \Phi^{H_{\varepsilon}}-\tau p(t)}=(D F)^{-t} e^{-\tau p(t)} .
$$

Clearly, this is maximized when $\tau=1$ and $D F=2$, i.e. at a point in $Y \cap f^{-1}(Y)$. We will maximize this by minimizing its reciprocal []$^{6}$ i.e. $e^{g_{\gamma}(t)}$,

where

$$
g_{\gamma}(t)=t \log 2+p_{\gamma}(t)
$$

We have added the subscript $\gamma$ to the expression for the pressure $p(t)$ to emphasize its dependence on $\gamma$. We proceed to minimize $g_{\gamma}(t)$ over $\gamma \in(0,1)$ and $t \in[0,1]$.

Note that $g_{\gamma}(t)$ is strictly convex with $g_{\gamma}(0)=\log 2=g_{\gamma}(1)$, for all $\gamma \in(0,1)$, so that its minimum occurs at an interior point of $[0,1]$. We will find the minimum of $g_{\gamma}$ by finding the point of intersection of two lines that lie below it: lower bounds on the tangent lines to $g_{\gamma}$ at $t=0$ and $t=1$.

At $t=1, g_{\gamma}^{\prime}(1)=\log 2-\chi_{\gamma}\left(\mu_{1}\right)>0$ where $\chi_{\gamma}\left(\mu_{1}\right)$ is the positive Lyapunov exponent with respect to the SRB measure $\mu_{1}$ for $f=f_{\gamma}$. Note that $\chi_{\gamma}\left(\mu_{1}\right) \downarrow 0$ as $\gamma \uparrow 1$ so we take as a lower bound for

\footnotetext{
${ }^{6}$ To prove Sublemma 6.5 for the potential $\Psi_{1, \varepsilon}^{H}$, one must instead minimize, $2^{t} e^{p^{H_{\varepsilon}}(t)+P_{t, \varepsilon}}$. But observe that $0=P(t \Phi-\tau p(t))=P\left(t \Phi-\tau p^{H_{\varepsilon}}(t)+\tau\left(p^{H_{\varepsilon}}(t)-p(t)\right)\right) \leqslant p^{H_{\varepsilon}}(t)-p(t)+P\left(t \Phi-\tau p^{H_{\varepsilon}}(t)\right)$

since $\tau \geqslant 1$. Thus $P_{t, \varepsilon} \geqslant p(t)-p^{H_{\varepsilon}}(t)$ and so $2^{t} e^{p^{H_{\varepsilon}}(t)+P_{t, \varepsilon}} \geqslant e^{g_{\gamma}(t)}$, and the estimate reduces to the current estimate for $\Psi_{2}^{H_{\varepsilon}}$.
} 
this tangent line, the line $u=t \log 2$. This line lies below $g_{\gamma}$ and $u(1)=\log 2=g_{\gamma}(1)$. Although $u(t)$ is not tangent to $g_{\gamma}(t)$, it is the limit of tangent lines at $t=1$ as $\gamma \rightarrow 1$.

At $t=0, g_{\gamma}^{\prime}(0)=\log 2-\chi_{\gamma}\left(\mu_{0}\right)<0$, where $\mu_{0}$ is the measure of maximal entropy for $f$. We proceed to derive an upper bound for $\chi_{\gamma}\left(\mu_{0}\right)$ that is independent of $\gamma \in(0,1)$.

Let $a_{\gamma}:=f_{L}^{-1}(1 / 2)$, where $f_{L}$ denotes the left branch of $f=f_{\gamma}$. We will use the fact that $\mu_{0}$ gives equal weight to all two-cylinders of the partition $\{[0,1 / 2),[1 / 2,1]\}$, i.e.,

$$
\mu_{0}\left(\left[0, a_{\gamma}\right]\right)=\mu_{0}\left(\left[a_{\gamma}, 1 / 2\right]\right)=\mu_{0}([1 / 2,3 / 4])=\mu_{0}([3 / 4,1])=1 / 4 \text {. }
$$

We want to maximize

$$
\chi_{\gamma}\left(\mu_{0}\right)=\int_{\left[0, a_{\gamma}\right]} \log \left|D f_{\gamma}\right| d \mu_{0}+\int_{\left[a_{\gamma}, 1 / 2\right]} \log \left|D f_{\gamma}\right| d \mu_{0}+\int_{[1 / 2,1]} \log \left|D f_{\gamma}\right| d \mu_{0} .
$$

The last integral above simply equals $\frac{1}{2} \log 2$. For the first two integrals, notice that $D f_{\gamma}(x)$ is strictly increasing for $x \in[0,1 / 2]$, so that for all $x \in\left[a_{\gamma}, 1 / 2\right]$ and all $\gamma \in(0,1)$,

$$
D f_{\gamma}(x) \leqslant D f_{\gamma}(1 / 2)=2+\gamma \leqslant 3=D f_{1}(1 / 2) \text {. }
$$

On the other hand, for $x \in\left[0, a_{\gamma}\right]$,

$$
D f_{\gamma}(x) \leqslant D f_{\gamma}\left(a_{\gamma}\right)=1+(1+\gamma)\left(2 a_{\gamma}\right)^{\gamma} .
$$

We claim that this expression is increasing in $\gamma$ and so is maximized when $\gamma=1$.

Claim. $\sup _{\gamma \in(0,1)} D f_{\gamma}\left(a_{\gamma}\right)=D f_{1}\left(a_{1}\right)=\sqrt{5}$.

Postponing the proof of the claim and applying these observations to (6.6), we have the following upper bound for $\chi_{\gamma}\left(\mu_{0}\right)$,

$$
\sup _{\gamma \in(0,1)} \chi_{\gamma}\left(\mu_{0}\right) \leqslant \frac{\log \sqrt{5}}{4}+\frac{\log 3}{4}+\frac{\log 2}{2}=\frac{\log (12 \sqrt{5})}{4} .
$$

Thus the slope $g_{\gamma}^{\prime}(0) \geqslant \log 2-\frac{\log (12 \sqrt{5})}{4}$ independently of $\gamma$. This implies that the minimum of $g_{\gamma}(t)$ will be at least as large as the point of intersection between $u(t)$ and this lower bound for the tangent line to $g_{\gamma}(t)$ at $t=0$. This occurs when

$$
\begin{aligned}
t \log 2 & =\log 2+t\left(\log 2-\frac{1}{4} \log (12 \sqrt{5})\right) \\
\Longrightarrow \log 2 & =\frac{t}{4} \log (12 \sqrt{5}) \Longrightarrow t=\frac{4 \log 2}{\log (12 \sqrt{5})} .
\end{aligned}
$$

Thus

and so

$$
\inf _{t \in[0,1]} g_{\gamma}(t) \geqslant \frac{4(\log 2)^{2}}{\log (12 \sqrt{5})}
$$

$$
e^{G} \leqslant e^{-2 g_{\gamma}} \leqslant e^{-\frac{8(\log 2)^{2}}{\log (12 \sqrt{5})}}<\frac{1}{3.216}
$$

for all $\gamma \in(0,1)$, completing the proof of the sublemma.

Proof of the Claim. Note that $a_{\gamma}$ by definition satisfies the following relation,

$$
f_{\gamma}\left(a_{\gamma}\right)=a_{\gamma}+2^{\gamma} a_{\gamma}^{\gamma+1}=\frac{1}{2} \Longrightarrow\left(2 a_{\gamma}\right)^{\gamma}=\frac{1}{2 a_{\gamma}}-1 .
$$

For $\gamma \in(0,1)$, we want to maximize

$$
M(\gamma):=D f_{\gamma}\left(a_{\gamma}\right)=1+(1+\gamma)\left(2 a_{\gamma}\right)^{\gamma}=1+(1+\gamma)\left(\frac{1}{2 a_{\gamma}}-1\right)=\frac{\gamma+1}{2 a_{\gamma}}-\gamma
$$


where we have used (6.8) to simplify the expression. Differentiating with respect to $\gamma$ we obtain,

$$
M^{\prime}(\gamma)=\frac{2 a_{\gamma}-(1+\gamma) 2 a_{\gamma}^{\prime}}{4 a_{\gamma}^{2}}-1=\frac{1}{2 a_{\gamma}}\left(1-\frac{(1+\gamma) a_{\gamma}^{\prime}}{a_{\gamma}}-2 a_{\gamma}\right),
$$

where $a_{\gamma}^{\prime}=\frac{d a_{\gamma}}{d \gamma}>0$. In order to eliminate $a_{\gamma}^{\prime}$, we differentiate (6.8) with respect to $\gamma$ to obtain,

$$
a_{\gamma}^{\prime}\left(1+\left(2 a_{\gamma}\right)^{\gamma}\right)+a_{\gamma}\left(2 a_{\gamma}\right)^{\gamma}\left[\log \left(2 a_{\gamma}\right)+\gamma \frac{a_{\gamma}^{\prime}}{a_{\gamma}}\right]=0 \quad \Longrightarrow \quad \frac{a_{\gamma}^{\prime}}{a_{\gamma}}=\frac{-\log \left(2 a_{\gamma}\right)}{\frac{1}{\left(2 a_{\gamma}\right)^{\gamma}}+1+\gamma} .
$$

Substituting this expression into 6.9 yields,

$$
M^{\prime}(\gamma)=\frac{1}{2 a_{\gamma}}\left(1+\frac{(1+\gamma) \log \left(2 a_{\gamma}\right)}{1+\gamma+\frac{1}{\left(2 a_{\gamma}\right)^{\gamma}}}-2 a_{\gamma}\right) \geq \frac{1}{2 a_{\gamma}}\left(1+\frac{(1+\gamma) \log \left(2 a_{\gamma}\right)}{2+\gamma}-2 a_{\gamma}\right),
$$

where we have used the fact that $\left(2 a_{\gamma}\right)^{\gamma} \leqslant 1$ and $\log \left(2 a_{\gamma}\right)<0$ to obtain the lower bound for $M^{\prime}(\gamma)$. To show that $M^{\prime}(\gamma)>0$, it suffices to show that the expression

$$
h(\gamma):=1+\frac{(1+\gamma) \log \left(2 a_{\gamma}\right)}{2+\gamma}-2 a_{\gamma}
$$

remains positive for $\gamma \in(0,1)$. Differentiating again, we obtain

$$
\begin{aligned}
h^{\prime}(\gamma) & =\frac{(2+\gamma)\left[\log \left(2 a_{\gamma}\right)+(1+\gamma) \frac{a_{\gamma}^{\prime}}{a_{\gamma}}\right]-(1+\gamma) \log \left(2 a_{\gamma}\right)}{(2+\gamma)^{2}}-2 a_{\gamma}^{\prime} \\
& =\frac{\log \left(2 a_{\gamma}\right)+(1+\gamma)(2+\gamma) \frac{a_{\gamma}^{\prime}}{a_{\gamma}}-2 a_{\gamma}(2+\gamma)^{2} \frac{a_{\gamma}^{\prime}}{a_{\gamma}}}{(2+\gamma)^{2}} \\
& =\frac{-\log \left(2 a_{\gamma}\right)}{(2+\gamma)^{2}}\left[-1+\frac{(1+\gamma)(2+\gamma)-2 a_{\gamma}(2+\gamma)^{2}}{\left.1+\gamma+\frac{1}{\left(2 a_{\gamma}\right)^{\gamma}}\right],}\right.
\end{aligned}
$$

where we have used 6.10 in the last line. Since $-\log \left(2 a_{\gamma}\right)>0$, it suffices to determine the sign of the expression in square brackets above. Now we use the fact that $a_{\gamma} \geqslant 1 / 4$ (attained when $\gamma=0$ ) and $\left(2 a_{\gamma}\right)^{\gamma}<1$ to write,

$$
\begin{aligned}
-1-\gamma-\frac{1}{\left(2 a_{\gamma}\right)^{\gamma}} & +(1+\gamma)(2+\gamma)-2 a_{\gamma}(2+\gamma)^{2} \\
& <-2-\gamma+2+3 \gamma+\gamma^{2}-2-2 \gamma-\frac{\gamma^{2}}{2}=-2+\frac{\gamma^{2}}{2} \leqslant-\frac{3}{2}<0 .
\end{aligned}
$$

We conclude that $h^{\prime}(\gamma)<0$ so that the minimum of $h$ occurs at $h(1)$. Since $a_{1}=\frac{\sqrt{5}-1}{4}$, we have

$$
h(1)=1+\frac{2}{3} \log \left(2 a_{1}\right)-2 a_{1}=\frac{3-\sqrt{5}}{2}+\frac{2}{3} \log \left(\frac{\sqrt{5}-1}{2}\right)>0 .
$$

Since $h(\gamma)$ is strictly positive, we conclude that $M^{\prime}(\gamma)$ is strictly positive and thus that $M(\gamma)$ attains its maximum at $\gamma=1$. Now $M(1)=D f_{1}\left(a_{1}\right)=\sqrt{5}$, completing the proof of the claim.

Proof of Proposition 6.2. Even without strict contraction, the estimates of Lemma 6.4 show that $\left\|{\stackrel{\mathcal{L}}{\Psi_{2}}}_{H_{\varepsilon}} \psi\right\|_{B V} \leqslant C\|\psi\|_{B V}$ for any $\psi \in B V(Y)$. This, together with the fact that $\left|\dot{\mathcal{L}}_{\Psi_{2}^{H_{\varepsilon}}} \psi\right|_{L^{1}\left(m_{t}\right)} \leqslant$ $|\psi|_{L^{1}\left(m_{t}\right)}$ implies that for any $n \in \mathbb{N}$ and $\psi \in B V(Y)$,

$$
\left\|{\stackrel{\mathcal{L}}{\Psi_{2}}}_{\Psi_{\varepsilon}}^{H_{\varepsilon}} \psi\right\|_{B V} \leqslant C\left(\sigma^{n / 2}\|\psi\|_{B V}+\frac{C_{3}}{1-\sigma}|\psi|_{L^{1}\left(m_{t}\right)}\right)
$$

for a uniform constant $C$, independent of $H_{\varepsilon}$. This is the standard Lasota-Yorke inequality. This inequality, together with the compactness of the unit ball of $B V(Y)$ in $L^{1}\left(m_{t}\right)$, implies that the essential spectral radius of ${\stackrel{\mathcal{L}}{\Psi_{2}^{H_{\varepsilon}}}}$ on $B V(Y)$ is bounded by $\sigma^{1 / 2}$. 
6.2. Perturbation Results. In this section, we will prove the following result.

Proposition 6.6. Let $\left(H_{\varepsilon}\right)_{\varepsilon \leqslant \varepsilon_{0}}$ be a family of nested intervals satisfying $\mathbf{( H )}$. Then for each $t \in$ $[0,1]$ and $\varepsilon$ sufficiently small, $\stackrel{\circ}{\mathcal{L}}_{\Psi_{1, \varepsilon}^{H}}=\stackrel{\circ}{\mathcal{L}}_{t \Phi^{H_{\varepsilon}}-\tau p^{H \varepsilon}(t)-P_{t, \varepsilon}}$ has a spectral gap on $B V(Y)$ equipped with the $\|\cdot\|_{B V}$ norm.

Indeed, the spectrum of ${\stackrel{\mathcal{L}}{\Psi_{1, \varepsilon}^{H}}}$ outside the disk of radius $\sigma^{1 / 2}$ is Hölder continuous in $\varepsilon$ and the spectral projectors vary Hölder continuously in the $|\cdot|_{L^{1}\left(m_{t}\right)}$ norm.

Proof. Since on the one hand, $m_{t}$ is not conformal with respect to the potential $\Psi_{1, \varepsilon}^{H}$ while on the other, the conformal measures $\tilde{m}_{t, H_{\varepsilon}}$ depend on $H_{\varepsilon}$, we will prove this proposition in two steps. First, notice that since $F$ is a full-branched Gibbs-Markov map, the unpunctured operator $\mathcal{L}_{\Psi_{2}}=\mathcal{L}_{t \Phi-\tau p(t)}$ enjoys a spectral gap on $B V(Y)$ equipped with the $\|\cdot\|_{B V}$ norm since the potential $\Psi_{2}$ is contracting in the sense of [LSV1]. We will show that the punctured transfer operator ${\stackrel{\mathcal{L}}{\Psi_{2}^{H_{\varepsilon}}}}=$ ${\stackrel{\circ}{\mathcal{L}_{t \Phi} H_{\varepsilon}-\tau p(t)}}$ is a perturbation of $\mathcal{L}_{\Psi_{2}}$ using the framework of [KL1] to conclude that this spectral gap persists for the punctured transfer operator for sufficiently small holes under assumption $(\mathbf{H})$. Indeed, it will follow that the spectral gap enjoyed by ${\stackrel{\circ}{\mathcal{L}_{t \Phi} H_{\varepsilon}-\tau p(t)}}$ has a lower bound that is uniform in $\varepsilon$. Second, we will show that the punctured transfer operator ${\stackrel{\circ}{\mathcal{L}_{\Psi_{1, \varepsilon}^{H}}}}=\stackrel{\circ}{\mathcal{L}}_{t \Phi^{H \varepsilon}-\tau p^{H \varepsilon}(t)-P_{t, \varepsilon}}$ is a perturbation of $\stackrel{\circ}{\mathcal{L}}_{t \Phi^{H_{\varepsilon}}-\tau p(t)}$ in a strong sense in $B V(Y)$. This will imply that for sufficiently small $\varepsilon, \stackrel{\circ}{\mathcal{L}}_{\Psi_{1, \varepsilon}^{H}}$ enjoys a spectral gap as well.

Step 1. In this step, we will prove that the spectra of ${\stackrel{\circ}{\mathcal{L}^{H_{\varepsilon}}}}={\stackrel{\circ}{\mathcal{L}_{t \Phi} H_{\varepsilon}-\tau p(t)}}$ and $\mathcal{L}_{\Psi_{2}}=\mathcal{L}_{t \Phi-\tau p(t)}$ are close in the sense of [KL1]. To this end, for two operators $P_{1}, P_{2}$ from $B V(Y)$ to $L^{1}\left(m_{t}\right)$, define the following norm.

$$
\left\|\left|P_{1} \psi-P_{2} \psi\right|\right\|=\sup \left\{\left|P_{1} \psi-P_{2} \psi\right|_{L^{1}\left(m_{t}\right)}:\|\psi\|_{B V} \leqslant 1\right\} .
$$

We begin with the following lemma.

Lemma 6.7. Let $H$ be a hole in $I$ and let $\tilde{H}$ be the induced hole for the map $F$ in $Y$. Then

$$
\left\|\left|\mathcal{L}_{\Psi_{2}}-\stackrel{\circ}{\mathcal{L}}_{\Psi_{2}^{H}}\right|\right\| \leqslant m_{t}\left(\tilde{H} \cup F^{-1}(\tilde{H})\right) .
$$

Proof. Let $\psi \in B V(Y),\|\psi\|_{B V} \leqslant 1$. Then in particular, $|\psi|_{\infty} \leqslant 1$. So,

$$
\left|\mathcal{L}_{\Psi_{2}} \psi-{\stackrel{\circ}{\mathcal{L}_{\Psi_{2}^{H}}}} \psi\right|_{L^{1}\left(m_{t}\right)}=\int\left|\mathcal{L}_{\Psi_{2}}\left(1_{\tilde{H} \cup F^{-1}(\tilde{H})} \psi\right)\right| d m_{t} \leqslant \int_{\tilde{H} \cup F^{-1}(\tilde{H})}|\psi| d m_{t} \leqslant m_{t}\left(\tilde{H} \cup F^{-1}(\tilde{H})\right) .
$$

For a family of holes satisfying $\mathbf{( H )}$, since $m_{t}\left(\tilde{H}_{\varepsilon} \cup F^{-1}\left(\tilde{H}_{\varepsilon}\right)\right) \rightarrow 0$ as $\varepsilon \rightarrow 0$ and using Proposition 6.2 and Lemma 6.7, it follows from [KL1, Corollary 1] that the spectrum and spectral projectors corresponding to eigenvalues outside the disk of radius $\sigma^{1 / 2}$ vary Holder continuously in the size of the perturbation.

Since $\mathcal{L}_{\Psi_{2}}$ has spectral radius 1 and enjoys a spectral gap, let $\bar{\beta}_{t, 0}<1$ denote the magnitude of its second largest eigenvalue. Letting $\bar{\Lambda}_{t, \varepsilon}$ and $\bar{\beta}_{t, \varepsilon}$ denote the largest and second largest eigenvalues of $\dot{\mathcal{L}}_{\Psi_{2}^{H_{\varepsilon}}}$, respectively, we conclude that both vary continuously in $\varepsilon$ for $\varepsilon$ sufficiently small. In particular, we may choose $\varepsilon_{0}$ sufficiently small that $\bar{\Lambda}_{t, \varepsilon}-\bar{\beta}_{t, \varepsilon}>\left(1-\bar{\beta}_{t, 0}\right) / 2$ for all $\varepsilon \leqslant \varepsilon_{0}$, i.e. ${\stackrel{\mathcal{L}}{\Psi^{H}}}_{2}^{H_{\varepsilon}}$ has a spectral gap. 


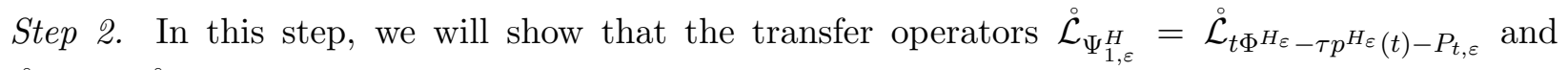
$\stackrel{\circ}{\mathcal{L}}_{\Psi_{2}^{H_{\varepsilon}}}=\stackrel{\circ}{\mathcal{L}}_{t \Phi^{H_{\varepsilon}}-\tau p(t)}$ are close in the $\|\cdot\|_{B V}$ norm.

Lemma 6.8. Suppose $H=H_{\varepsilon}$ belongs to a family of holes satisfying assumption ( $\left.H\right)$. Then there exists $C>0$, independent of $\varepsilon$, such that

$$
\left\|{\stackrel{\circ}{\Psi^{H}}}_{1, \varepsilon}-\stackrel{\circ}{\mathcal{L}}_{\Psi_{2}^{H}}\right\|_{B V} \leqslant C\left(p(t)-p^{H_{\varepsilon}}(t)+P_{t, \varepsilon}\right),
$$

where $\|\psi\|_{B V}=\bigvee_{Y} \psi+|\psi|_{L^{1}\left(m_{t}\right)}$.

Proof. Let $Z_{n}^{i}=\left(a_{n}^{i}, b_{n}^{i}\right), i=1,2$, denote the at most two maximal intervals in $Y$ on which $\stackrel{\circ}{F}$ is monotonic and continuous with $\left.\tau\right|_{Z_{n}^{i}}=n$. Letting $\xi_{n}^{i}$ denote the inverse of $\left.F\right|_{Z_{n}^{i}}$, for $\psi \in B V(Y)$ we follow (6.3),

$$
\begin{aligned}
\bigvee_{Y}\left(\mathcal{L}_{\Psi_{1, \varepsilon}^{H}} \psi-\mathcal{L}_{\Psi_{2}^{H_{\varepsilon}}} \psi\right) \leqslant & \sum_{n, i} \bigvee_{Y} \psi \circ \xi_{n}^{i}\left(e^{\Psi_{1, \varepsilon} \circ \xi_{n}^{i}}-e^{\Psi_{2} \circ \xi_{n}^{i}}\right) \\
& +\sum_{n, i}\left|\psi\left(a_{n}^{i}\right)\right|\left|e^{\Psi_{1, \varepsilon}\left(a_{n}^{i}\right)}-e^{\Psi_{2}\left(a_{n}^{i}\right)}\right|+\left|\psi\left(b_{n}^{i}\right)\right|\left|e^{\Psi_{1, \varepsilon}\left(b_{n}^{i}\right)}-e^{\Psi_{2}\left(b_{n}^{i}\right)}\right| \\
\leqslant & \sum_{n, i} \sup _{Z_{n}^{i}}\left|e^{\Psi_{1, \varepsilon}}-e^{\Psi_{2}}\right|\left(\bigvee_{Z_{n}^{i}} \psi+\sup _{Z_{n}^{i}} \psi\right)+\sum_{n, i} \sup _{Z_{n}^{i}}\left|e^{\Psi_{1, \varepsilon}}-e^{\Psi_{2}}\right|\left(\bigvee_{Z_{n}^{i}} \psi+\inf _{Z_{n}^{i}} \psi\right) \\
\leqslant & 4\|\psi\|_{B V} \sum_{n, i} \sup _{Z_{n}^{i}}\left|e^{\Psi_{1, \varepsilon}}-e^{\Psi_{2}}\right|,
\end{aligned}
$$

where we have used the fact that $e^{\Psi_{1, \varepsilon}}-e^{\Psi_{2}}$ is monotonic and does not change sign on each $Z_{n}^{i}$ to bound the variation by the supremum of the function. Fixing $Z_{n}^{i}$, we estimate,

$$
\begin{aligned}
\left|e^{\Psi_{1, \varepsilon}}-e^{\Psi_{2}}\right| & =e^{t \Phi-\tau p(t)}\left|1-e^{\left(p(t)-p^{H_{\varepsilon}}(t)\right) \tau-P_{t, \varepsilon}}\right| \\
& \leqslant C n^{-t\left(1+\frac{1}{\gamma}\right)} e^{-n p(t)}\left(n\left(p(t)-p^{H_{\varepsilon}}(t)\right)+P_{t, \varepsilon}\right) e^{\left(p(t)-p^{H_{\varepsilon}}(t)\right) n+P_{t, \varepsilon}} \\
& \leqslant C n^{-t\left(1+\frac{1}{\gamma}\right)+1} e^{-n p^{H_{\varepsilon}}(t)}\left(p(t)-p^{H_{\varepsilon}}(t)+P_{t, \varepsilon}\right),
\end{aligned}
$$

where in the second line we have used the estimate $\left|1-e^{x}\right| \leqslant x e^{x}$ for $x \geqslant 0$ and $\left|1-e^{x}\right| \leqslant|x|$ for $x<0$.

Summing over $n$, we see that the sum is bounded uniformly in $\varepsilon$ since $p^{H_{\varepsilon}}(t)>0$ for $t<t^{H_{\varepsilon}}$ and since $t^{H_{\varepsilon}}>2 \gamma /(1+\gamma)$ by assumption of 6.1 , we have $\sum_{n} n^{1-t\left(1+\frac{1}{\gamma}\right)}<\infty$ for $t \geqslant t^{H_{\varepsilon}}$. We have also used the fact that there are at most two $Z_{n}^{i}$ per $n \in \mathbb{N}$.

To bound the difference in $L^{1}\left(m_{t}\right)$ norm for $\psi \in B V(Y)$, we use the fact that $m_{t}$ is conformal with respect to $\Psi_{2}$ to write

$$
\begin{aligned}
\int_{Y}\left|\mathcal{L}_{\Psi_{1, \varepsilon}^{H}} \psi-\mathcal{L}_{\Psi_{2}^{H_{\varepsilon}}} \psi\right| d m_{t} & =\int_{\dot{Y}_{\varepsilon}^{1}}|\psi|\left|1-e^{\left(p(t)-p^{H_{\varepsilon}}(t)\right) \tau-P_{t, \varepsilon}}\right| \\
& \leqslant C\|\psi\|_{B V} \sum_{n, i} m_{t}\left(Z_{n}^{i}\right)\left|1-e^{n\left(p(t)-p^{H_{\varepsilon}}(t)\right)-P_{t, \varepsilon}}\right|
\end{aligned}
$$

and note that this is the same estimate as above since due to conformality and the large images assumption $(\mathrm{H}), m_{t}\left(Z_{n}^{i}\right)$ is proportional to $e^{t \Phi-\tau p(t)}$.

Our next lemma shows that in fact the bound obtained in the previous lemma is continuous in $\varepsilon$. 
Lemma 6.9. Let $\left(H_{\varepsilon}\right)_{\varepsilon \leqslant \varepsilon_{0}}$ be a collection of holes centered at $z \in I$ with $H_{\varepsilon} \rightarrow\{z\}$ as $\varepsilon \rightarrow 0$. Then for $t \in[0,1]$,

$$
p^{H_{\varepsilon}}(t) \rightarrow p(t), \quad t^{H_{\varepsilon}} \rightarrow 1 \text { and } P_{t, \varepsilon} \rightarrow 0 \quad \text { as } \varepsilon \rightarrow 0 .
$$

Proof. Note that for $t=1$, the statement of the lemma is trivial since $p^{H_{\varepsilon}}(1)=p(1)=0$ and so $P_{1, \varepsilon}=-\log \bar{\Lambda}_{1, \varepsilon}$ is continuous in $\varepsilon$ by Step 1. We now focus on $t<1$. Since the quantities of interest are clearly monotone in the size of the hole, we need only prove the lemma for Markov holes.

By Corollary 1.8, we have $\log \lambda_{t}^{H_{\varepsilon}}=p^{H_{\varepsilon}}(t)-p(t)$. By 4.23) and the comment following it, we have $\lambda_{t}^{H_{\varepsilon}} \rightarrow 1$ as $\varepsilon \rightarrow 0$. Thus $p^{H_{\varepsilon}}(t) \rightarrow p(t)$ as $\varepsilon \rightarrow 0$.

Now fix $t<1$. Since $p(t)>0$, by the previous paragraph we may choose $\varepsilon>0$ sufficiently small such that $p^{H_{\varepsilon}}(t)>0$. By Proposition 1.6, this implies $t^{H_{\varepsilon}}>t$ and by monotonicity, $t^{H_{\varepsilon^{\prime}}}>t$ for all $\varepsilon^{\prime} \leqslant \varepsilon$. Since this is true for each $t<1$, we have $t^{H_{\varepsilon}} \rightarrow 1$ as $\varepsilon \rightarrow 0$.

Finally, consider the rescaled transfer operator $e^{P_{t, \varepsilon}} \mathcal{L}_{\Psi_{1, \varepsilon}}=\mathcal{L}_{t \Phi-\tau p^{H_{\varepsilon}}(t)}$ whose spectral radius on $B V(Y)$ is $e^{P_{t, \varepsilon}}$. Replacing $\mathcal{L}_{\Psi_{1, \varepsilon}}$ by this rescaled operator in the statement and proof of Lemma 6.8 yields,

$$
\left\|\mathcal{L}_{t \Phi-\tau p^{H_{\varepsilon}(t)}}-\mathcal{L}_{t \Phi-\tau p(t)}\right\|_{B V} \leqslant C\left(p(t)-p^{H_{\varepsilon}}(t)\right) .
$$

Using now that $p^{H_{\varepsilon}}(t) \rightarrow p(t)$ as $\varepsilon \rightarrow 0$ and the fact that $\mathcal{L}_{t \Phi-\tau p(t)}$ has a spectral gap with leading eigenvalue 1 , we conclude using standard perturbation theory that the leading eigenvalue of $\mathcal{L}_{t \Phi-\tau p^{H \varepsilon}(t)}$ tends to 1 as $\varepsilon \rightarrow 0$. This implies $P_{t, \varepsilon} \rightarrow 0$ as required.

Since $P_{t, \varepsilon} \rightarrow 0$ and $p^{H_{\varepsilon}}(t) \rightarrow p(t)$ as $\varepsilon \rightarrow 0$, Lemmas 6.8 and 6.9 imply that as operators on $B V(Y),{\stackrel{\mathcal{L}}{\Psi_{1, \varepsilon}^{H}}}$ and ${\stackrel{\mathcal{L}}{\Psi_{2}^{H}}}_{\varepsilon}$ are close so that their spectra and spectral projectors vary continuously by standard perturbation theory (see $[\mathrm{K}]$ ).

Thus for $\varepsilon$ sufficiently small, the largest eigenvalue of $\stackrel{\circ}{\mathcal{L}}_{\Psi_{1, \varepsilon}^{H}}, \Lambda_{t, \varepsilon}$, is close to $\bar{\Lambda}_{t, \varepsilon}$, while the second largest eigenvalue, $\beta_{t, \varepsilon}$ is as close as we like to $\bar{\beta}_{t, \varepsilon}$ (if it lies outside the disk of radius $\sigma^{1 / 2}$ ). Since by Step $1, \bar{\Lambda}_{t, \varepsilon}$ and $\bar{\beta}_{t, \varepsilon}$ are uniformly bounded away from one another for all $\varepsilon \leqslant \varepsilon_{0}$, we may further shrink $\varepsilon_{0}$ if necessary so that $\Lambda_{t, \varepsilon}$ and $\beta_{t, \varepsilon}$ are uniformly bounded away from one another for all $\varepsilon \leqslant \varepsilon_{0}$. Thus ${\stackrel{\circ}{\mathcal{L}_{1, \varepsilon}^{H}}}$ has a spectral gap on $B V(Y)$ for all $\varepsilon \leqslant \varepsilon_{0}$.

This completes the proof of Proposition 6.6.

6.3. An invariant measure for $\stackrel{\circ}{F}_{\varepsilon}$ on $\stackrel{\circ}{Y}_{\varepsilon}^{\infty}$. In this section, we fix $t \in[0,1]$ and assume that $\varepsilon_{0}$ is small enough that for each $H_{\varepsilon}$ with $\varepsilon \leqslant \varepsilon_{0}, \stackrel{\circ}{\mathcal{L}}_{\Psi_{1, \varepsilon}^{H}}=: \stackrel{\circ}{\mathcal{L}}_{\varepsilon}$ has a spectral gap by Proposition 6.6.

Thus for $\varepsilon \in\left(0, \varepsilon_{0}\right]$ there exists a maximal eigenvalue $\Lambda_{\varepsilon}<1$ for $\dot{\mathcal{L}}_{\varepsilon}$ and unique $g_{\varepsilon} \in B V(Y)$ on $\stackrel{\circ}{Y}_{\varepsilon}=Y \backslash \tilde{H}_{\varepsilon}$ such that $\dot{\mathcal{L}}_{\varepsilon} g_{\varepsilon}=\Lambda_{\varepsilon} g_{\varepsilon}$ and $g_{\varepsilon} \tilde{m}_{t, H_{\varepsilon}}$ defines a conditionally invariant probability measure for $\stackrel{\circ}{F}$ with escape rate $-\log \Lambda_{\varepsilon}$. Moreover, there exists $C>0$ and $\rho<1$ such that for each $\psi \in B V(Y)$ and $n \geqslant 0$, we have

$$
\left\|\Lambda_{\varepsilon}^{-n} \stackrel{\mathcal{L}}{\varepsilon}_{\varepsilon}^{n} \psi-e_{\varepsilon}(\psi) g_{\varepsilon}\right\|_{B V} \leqslant C\|\psi\|_{B V} \rho^{n},
$$

where $e_{\varepsilon}(\psi)$ is determined by the spectral projector $\Pi_{\varepsilon}$ of $\stackrel{\circ}{\mathcal{L}}_{\varepsilon}$ onto the subspace spanned by $g_{\varepsilon}$ : $e_{\varepsilon}(\psi)=\left|\Pi_{\varepsilon} \psi\right|_{L^{1}\left(\tilde{m}_{t, H}\right)}$, due to the normalization of $g_{\varepsilon}$ we have chosen. If $\psi$ is a probability density with respect to $m_{t}$, then $e_{0}(\psi)=1$. 
We define an invariant measure on the survivor set $\stackrel{\circ}{Y}_{\varepsilon}^{\infty}$ using a well-known construction. For $\psi \in B V$, define the functional,

$$
\nu_{\varepsilon}(\psi)=\lim _{n \rightarrow \infty} \Lambda_{\varepsilon}^{-n} \int_{\dot{Y}_{\varepsilon}^{n}} \psi g_{\varepsilon} d \tilde{m}_{t, H_{\varepsilon}}=\lim _{n \rightarrow \infty} \Lambda_{\varepsilon}^{-n} \int_{\dot{\Upsilon}_{\varepsilon}} \dot{\mathcal{L}}_{\varepsilon}^{n}\left(\psi g_{\varepsilon}\right) d \tilde{m}_{t, H_{\varepsilon}}=e_{\varepsilon}\left(\psi g_{\varepsilon}\right),
$$

so that the limit is well-defined on $B V$. Note that $\nu_{\varepsilon}$ is linear, positive and $\nu_{\varepsilon}(\psi) \leqslant|\psi|_{\infty}$ so that $\nu_{\varepsilon}$ can be extended to a bounded, positive linear functional on $C^{0}(Y)$. Since, $\nu_{\varepsilon}(1)=1$, by the Riesz representation theorem, $\nu_{\varepsilon}$ corresponds to a unique Borel probability measure. From its definition, it is clear that $\nu_{\varepsilon}$ is supported on the survivor set $\dot{Y}_{\varepsilon}^{\infty}$.

Proposition 6.10. Let $\left(H_{\varepsilon}\right)_{\varepsilon_{0}}$ be a family of holes as in Proposition 6.6 such that that $\varepsilon_{0}>0$ is small enough that $\mathcal{L}_{\varepsilon}$ has a spectral gap for each $\varepsilon \leqslant \varepsilon_{0}$. Let $\nu_{\varepsilon}$ be the corresponding invariant measure on the survivor set defined by (6.11). Then

$$
\nu_{\varepsilon}(\psi) \rightarrow \nu_{0}(\psi) \text { as } \varepsilon \rightarrow 0 \text { for each } \psi \in C^{0}(Y) \cup B V(Y),
$$

where $\nu_{0}$ is the unique invariant measure absolutely continuous with respect to $m_{t}$ for $F$, the induced map without the hole.

Proof. Note that for $\varepsilon>0, \nu_{\varepsilon}$ is singular with respect to $m_{t}$ for $\varepsilon>0$, but that $d \nu_{0}=g_{0} d m_{t}$ where $g_{0} \in B V(Y)$ is the unique invariant probability density for $\mathcal{L}_{t \Phi-\tau p(t)} .^{7}$

Let $\bar{g}_{\varepsilon} d m_{t}, \bar{g}_{\varepsilon} \in B V(Y)$, denote the conditionally invariant probability measure formed using the eigenvector $\bar{g}_{\varepsilon}$ for $\dot{\mathcal{L}}_{\Psi_{2}^{H_{\varepsilon}}}$, and let $\bar{\nu}_{\varepsilon}$ denote the invariant measure on the survivor set $Y_{\varepsilon}^{\infty}$ formed via the limit in 6.11$)^{2}$, but using $\bar{g}_{\varepsilon} d m_{t}$ in place of $g_{\varepsilon} d \tilde{m}_{t, H_{\varepsilon}}$.

For $\psi \in B V(Y)$, we have

$$
\left|\nu_{\varepsilon}(\psi)-\nu_{0}(\psi)\right| \leqslant\left|\nu_{\varepsilon}(\psi)-\bar{\nu}_{\varepsilon}(\psi)\right|+\left|\bar{\nu}_{\varepsilon}(\psi)-\nu_{0}(\psi)\right| .
$$

To estimate the first term above, we write

$$
\left|\nu_{\varepsilon}(\psi)-\bar{\nu}_{\varepsilon}(\psi)\right| \leqslant\left|e_{\varepsilon}\left(\psi g_{\varepsilon}\right)-\bar{e}_{\varepsilon}\left(\psi g_{\varepsilon}\right)\right|+\left|\bar{e}_{\varepsilon}\left(\psi g_{\varepsilon}\right)-\bar{e}_{\varepsilon}\left(\psi \bar{g}_{\varepsilon}\right)\right|
$$

Let $\Pi_{\varepsilon}$ and $\bar{\Pi}_{\varepsilon}$ denote the projectors onto the eigenspaces corresponding to the top eigenvalues of $\stackrel{\circ}{\mathcal{L}}_{\Psi_{1, \varepsilon}^{H}}$ and $\stackrel{\circ}{\mathcal{L}}_{\Psi_{2}^{H}}$, respectively, so that

$$
\Pi_{\varepsilon}\left(\psi g_{\varepsilon}\right)=e_{\varepsilon}\left(\psi g_{\varepsilon}\right) g_{\varepsilon} \text { and } \bar{\Pi}_{\varepsilon}\left(\psi g_{\varepsilon}\right)=\bar{e}_{\varepsilon}\left(\psi g_{\varepsilon}\right) \bar{g}_{\varepsilon}
$$

By Lemma 6.8, $\left\|g_{\varepsilon}-\bar{g}_{\varepsilon}\right\|_{B V} \leqslant \zeta(\varepsilon)$ for some function $\zeta$ such that $\zeta(\varepsilon) \rightarrow 0$ as $\varepsilon \rightarrow 0$. Similarly, $\left\|\Pi_{\varepsilon}-\bar{\Pi}_{\varepsilon}\right\|_{B V} \leqslant \zeta(\varepsilon)$.

$$
\begin{aligned}
\| \Pi_{\varepsilon}\left(\psi g_{\varepsilon}\right) & -\bar{\Pi}_{\varepsilon}\left(\psi g_{\varepsilon}\right) \|_{B V} \geqslant \int\left|\Pi_{\varepsilon}\left(\psi g_{\varepsilon}\right)-\bar{\Pi}_{\varepsilon}\left(\psi g_{\varepsilon}\right)\right| d m_{t} \\
& =\left|\int\left(e_{\varepsilon}\left(\psi g_{\varepsilon}\right)-\bar{e}_{\varepsilon}\left(\psi g_{\varepsilon}\right)\right) g_{\varepsilon} d m_{t}+\bar{e}_{\varepsilon}\left(\psi g_{\varepsilon}\right) \int\left(g_{\varepsilon}-\bar{g}_{\varepsilon}\right) d m_{t}\right|
\end{aligned}
$$

Since the term on the left of the inequality is of order $\zeta(\varepsilon)$ and the second term on the right is of the same order, then the same must be true of the first term on the right. It follows then that $e_{\varepsilon}(\cdot)$ and $\bar{e}_{\varepsilon}(\cdot)$ can be made arbitrarily close on $B V$ functions by choosing $\varepsilon$ small. Thus the first term in 6.13 can be made arbitrarily small by choosing $\varepsilon$ small.

For the second term of 6.13 , we estimate,

$$
\left|\bar{e}_{\varepsilon}\left(\psi g_{\varepsilon}\right)-\bar{e}_{\varepsilon}\left(\psi \bar{g}_{\varepsilon}\right)\right|=\left|\int \bar{\Pi}_{\varepsilon}\left(\psi g_{\varepsilon}-\psi \bar{g}_{\varepsilon}\right) d m_{t}\right| \leqslant C\|\psi\|_{B V}\left\|g_{\varepsilon}-\bar{g}_{\varepsilon}\right\|_{B V}
$$

\footnotetext{
${ }^{7}$ Since $H_{0}=\{z\}, p^{H_{0}}(t)=p(t)$ so that the conformal measure for $\Psi_{1,0}^{H}$ is once again $m_{t}$.
} 
which can again be made arbitrarily small. This completes the estimate on the first term of 6.12. To estimate the second term in 6.12 , we use Lemma 6.7 so that the spectra and spectral proctors of $\stackrel{\circ}{\mathcal{L}}_{\Psi_{2}^{H_{\varepsilon}}}$ converge to those of $\mathcal{L}_{t \Phi-\tau p(t)}$ in the weaker $L^{1}\left(m_{t}\right)$ norm and not in $\|\cdot\|_{B V}$.

$$
\begin{aligned}
\left|\bar{\nu}_{\varepsilon}(\psi)-\nu_{0}(\psi)\right| & \leqslant\left|\bar{e}_{\varepsilon}\left(\psi \bar{g}_{\varepsilon}\right)-e_{0}\left(\psi \bar{g}_{\varepsilon}\right)\right|+\left|e_{0}\left(\psi \bar{g}_{\varepsilon}\right)-e_{0}\left(\psi g_{0}\right)\right| \\
& =\left|\int \bar{\Pi}_{\varepsilon}\left(\psi \bar{g}_{\varepsilon}\right) d m_{t}-\int \Pi_{0}\left(\psi \bar{g}_{\varepsilon}\right) d m_{t}\right|+\left|\int \psi\left(\bar{g}_{\varepsilon}-g_{0}\right) d m_{t}\right| \\
& \leqslant\left|\bar{\Pi}_{\varepsilon}\left(\psi \bar{g}_{\varepsilon}\right)-\Pi_{0}\left(\psi \bar{g}_{\varepsilon}\right)\right|_{L^{1}\left(m_{t}\right)}+\|\psi\|_{B V}\left|\bar{g}_{\varepsilon}-g_{0}\right|_{L^{1}\left(m_{t}\right)} \\
& \leqslant||\left|\bar{\Pi}_{\varepsilon}-\Pi_{0}\right|||\left\|\psi \bar{g}_{\varepsilon}\right\|_{B V}+\|\psi\|_{B V}\left|\bar{g}_{\varepsilon}-g_{0}\right|_{L^{1}\left(m_{t}\right)}
\end{aligned}
$$

The first term above tends to zero as $\varepsilon$ tends to 0 due to Lemma 6.7, while the second term tends to zero by [KL1, Corollary 1].

Putting these estimates together with $(6.13)$ in $(6.12)$ completes the proof of convergence of $\nu_{\varepsilon}$ to $\nu_{0}$ when integrated against functions in $B V(Y)$. We extend this convergence to continuous functions by approximation. For $\psi_{1} \in C^{0}(Y)$, let $\delta>0$ and choose a step function $\psi_{2} \in B V(Y)$ such that $\left|\psi_{1}-\psi_{2}\right|_{\infty}<\delta$. Then

$$
\begin{aligned}
\left|\nu_{\varepsilon}\left(\psi_{1}\right)-\nu_{0}\left(\psi_{1}\right)\right| & \leqslant\left|\nu_{\varepsilon}\left(\psi_{1}\right)-\nu_{\varepsilon}\left(\psi_{2}\right)\right|+\left|\nu_{\varepsilon}\left(\psi_{2}\right)-\nu_{0}\left(\psi_{2}\right)\right|+\left|\nu_{0}\left(\psi_{2}\right)-\nu_{0}\left(\psi_{1}\right)\right| \\
& \leqslant 2 \delta+\left|\nu_{\varepsilon}\left(\psi_{2}\right)-\nu_{0}\left(\psi_{2}\right)\right|,
\end{aligned}
$$

and the last term tends to 0 as $\varepsilon \rightarrow 0$ since $\psi_{2} \in B V(Y)$. Since $\delta>0$ was arbitrary, this implies the required convergence on continuous functions.

6.4. An invariant measure for $\stackrel{\circ}{f}_{\varepsilon}$ on $\stackrel{\circ}{I}_{\varepsilon}^{\infty}$. In this section, we push the invariant measure $\nu_{\varepsilon}=\nu_{Y, \varepsilon}$ on $\stackrel{\circ}{Y}_{\varepsilon}^{\infty}$ onto $\stackrel{\circ}{I}_{\varepsilon}^{\infty}$ to obtain an invariant measure $\nu_{H_{\varepsilon}}$ for $\stackrel{\circ}{\varepsilon}_{\varepsilon}$ which inherits good properties from $\nu_{Y, \varepsilon}$, thus completing parts (1) and (5) of Theorem 1.13 .

Define for any Borel set $A \subset I$,

$$
\nu_{H_{\varepsilon}}(A)=\frac{1}{\int \tau d \nu_{Y, \varepsilon}} \sum_{k=0}^{\infty} \sum_{i=0}^{\tau_{k}-1} \nu_{Y, \varepsilon}\left(f^{-i}(A) \cap Y_{k}\right),
$$

where $\tau_{k}=\left.\tau\right|_{Y_{k}}=k+1$. This defines an $f$-invariant probability measure $\nu_{H_{\varepsilon}}$ if $\int \tau d \nu_{Y, \varepsilon}<\infty$. The next lemma shows that in fact $\tau$ is uniformly integrable with respect to $\nu_{Y, \varepsilon}$ for all $\varepsilon$ sufficiently small.

Lemma 6.11. There exists a constant $C_{4}>0$ such that for each $k \geqslant 0$ and all $\varepsilon$ sufficiently small, $\nu_{Y, \varepsilon}\left(Y_{k}\right) \leqslant C_{4} k^{-t\left(\frac{1}{\gamma}+1\right)} e^{-(k+1) p^{H_{\varepsilon}}(t)}$.

Proof. Fix $k \geqslant 0$ and let $e^{\left(t \Phi-\tau p^{H_{\varepsilon}}(t)-P_{t, \varepsilon}\right)\left(Y_{k}\right)}$ denote the maximum of $e^{t \Phi-\tau p^{H_{\varepsilon}}(t)-P_{t, \varepsilon}}$ on $Y_{k}$. Since $F\left(Y_{k}\right)=[1 / 2,1]$, we have for $n \geqslant 1$,

$$
\begin{aligned}
\tilde{m}_{t, H_{\varepsilon}}\left(\stackrel{\circ}{Y}_{\varepsilon}^{n} \cap Y_{k}\right) & \leqslant e^{\left(t \Phi-\tau p^{H_{\varepsilon}}(t)-P_{t, \varepsilon}\right)} \tilde{m}_{t, H_{\varepsilon}}\left(\stackrel{\circ}{Y}_{\varepsilon}^{n-1} \cap F\left(Y_{k}\right)\right) \\
& \leqslant C_{5} k^{-t\left(\frac{1}{\gamma}+1\right)} e^{-(k+1) p^{H_{\varepsilon}}(t)} \tilde{m}_{t, H_{\varepsilon}}\left(\stackrel{\circ}{\varepsilon}_{\varepsilon}^{n-1}\right)
\end{aligned}
$$

for some uniform constant $C_{5}$ depending on bounded distortion. Note that $C_{5}$ is independent of $\varepsilon$ (and $t$ ). Now

$$
\Lambda_{\varepsilon}^{-n+1} \tilde{m}_{t, H_{\varepsilon}}\left(\stackrel{\circ}{\varepsilon}_{\varepsilon}^{n-1}\right)=\Lambda_{\varepsilon}^{-n+1} \int{\stackrel{\mathcal{L}^{n}}{\Psi_{1, \varepsilon}^{H}}}^{n-1} 1 d \tilde{m}_{t, H_{\varepsilon}} \underset{n \rightarrow \infty}{\longrightarrow} e_{\varepsilon}(1)>0
$$


Therefore, there exists $n_{1}=n_{1}(\varepsilon)>0$ such that $\Lambda_{\varepsilon}^{-n+1} \tilde{m}_{t, H_{\varepsilon}}\left(\dot{Y}_{\varepsilon}^{n-1}\right) \leqslant 2 e_{\varepsilon}(1)$ for all $n>n_{1}$. Also, since $e_{\varepsilon}(1) \rightarrow e_{0}(1)=1$ as $\varepsilon \rightarrow 0$, we have $e_{\varepsilon}(1) \leqslant 2$ for all $\varepsilon$ sufficiently small.

Putting these estimates together with 6.15 yields,

$$
\begin{aligned}
\nu_{\varepsilon}\left(Y_{k}\right) & =\lim _{n \rightarrow \infty} \Lambda_{\varepsilon}^{-n} \int_{\dot{Y}_{\varepsilon}^{n}} 1_{Y_{k}} g_{\varepsilon} d \tilde{m}_{t, H_{\varepsilon}} \leqslant \lim _{n \rightarrow \infty} \Lambda_{\varepsilon}^{-n}\left|g_{\varepsilon}\right|_{\infty} \tilde{m}_{t, H_{\varepsilon}}\left(\stackrel{\circ}{\varepsilon}_{\varepsilon}^{n} \cap Y_{k}\right) \\
& \leqslant \lim _{n \rightarrow \infty}\left|g_{\varepsilon}\right|_{\infty} \Lambda_{\varepsilon}^{-1} C_{5} k^{-t\left(\frac{1}{\gamma}+1\right)} e^{-(k+1) p^{H_{\varepsilon}}(t)} \Lambda_{\varepsilon}^{-n+1} \tilde{m}_{t, H_{\varepsilon}}\left(\stackrel{Y}{\varepsilon}_{\varepsilon}^{n-1}\right) \\
& \leqslant 4 \Lambda_{\varepsilon}^{-1} C_{5}\left|g_{\varepsilon}\right|_{\infty} k^{-t\left(\frac{1}{\gamma}+1\right)} e^{-(k+1) p^{H_{\varepsilon}}(t)} .
\end{aligned}
$$

Now since $g_{\varepsilon}$ lies in a uniform ball in $B V(Y)$ for all $\varepsilon$ sufficiently small, the lemma is proved since the constants are bounded independently of $\varepsilon$ and $k$.

As a consequence of Lemma 6.11, we have

$$
\int_{\tau>k_{0}} \tau d \nu_{Y, \varepsilon}=\sum_{k=k_{0}}^{\infty} \tau_{k} \nu_{Y, \varepsilon}\left(Y_{k}\right) \leqslant \sum_{k=k_{0}}^{\infty}(k+1) C_{4} k^{-t\left(\frac{1}{\gamma}+1\right)} e^{-(k+1) p^{H_{\varepsilon}}(t)} \leqslant \rho_{t}\left(k_{0}\right),
$$

where $\rho_{t}\left(k_{0}\right) \rightarrow 0$ as $k_{0} \rightarrow \infty$. In this last step, we have used the fact that $p^{H_{\varepsilon}}(t)>0$ for $t<t^{H}$ as well as the assumption that $t^{H_{\varepsilon}}>2 \gamma /(1+\gamma)$, to conclude that the tail of the series tends to 0 as $k_{0}$ increases.

Lemma 6.12. $\int \tau d \nu_{Y, \varepsilon} \rightarrow \int \tau d \nu_{Y, 0}$ as $\varepsilon \rightarrow 0$.

Proof. Fix $\delta>0$. By 6.16, we may choose $k_{0}$ so that $\int_{\tau>k_{0}} \tau d \nu_{Y, \varepsilon}<\delta$ for all $\varepsilon$ sufficiently small (including $\varepsilon=0$ ). Since $\tau$ is constant on each $Y_{k}$, we have $\tau \cdot 1_{\left\{\tau \leqslant k_{0}\right\}} \in B V(Y)$. Thus

$$
\left|\int \tau d \nu_{Y, \varepsilon}-\int \tau d \nu_{Y, 0}\right| \leqslant\left|\int \tau \cdot 1_{\left\{\tau \leqslant k_{0}\right\}} d \nu_{Y, \varepsilon}-\int \tau \cdot 1_{\left\{\tau \leqslant k_{0}\right\}} d \nu_{Y, 0}\right|+2 \delta
$$

and the difference of integrals on the right goes to zero as $\varepsilon \rightarrow 0$ by Proposition 6.10 . Since $\delta>0$ was arbitrary, this completes the proof of the lemma.

For part (5) of Theorem 1.13 , we need to show that $\nu_{H_{\varepsilon}} \rightarrow \mu_{t}$ as $\varepsilon \rightarrow 0$, where $\mu_{t}$ is the equilibrium state (absolutely continuous with respect to $m_{t}$ ) for the unpunctured potential $t \phi-p(t)$. This is straightforward with Lemmas 6.11 and 6.12 in hand since $\mu_{t}$ is simply the projection of $\nu_{Y, 0}$ to $I$ via the formula analogous to (6.14). Fix a function $\psi$ with bounded variation on $I$ and $\delta>0$. Choose $k_{0}$ such that $\int_{\tau>k_{0}} \tau d \nu_{Y, \varepsilon}<\delta$. Then

$$
\begin{aligned}
\left|\nu_{H_{\varepsilon}}(\psi)-\mu_{t}(\psi)\right| \leqslant & \left|\sum_{k=0}^{k_{0}} \sum_{i=0}^{\tau_{k}-1} \frac{\nu_{Y, \varepsilon}\left(\psi \circ f^{i} \cdot 1_{Y_{k}}\right)}{\int \tau d \nu_{Y, \varepsilon}}-\frac{\nu_{Y, 0}\left(\psi \circ f^{i} \cdot 1_{Y_{k}}\right)}{\int \tau d \nu_{Y, 0}}\right| \\
& +\|\psi\|_{B V} \sum_{k>k_{0}} \sum_{i=0}^{\tau_{k}-1} \frac{\nu_{Y, \varepsilon}\left(Y_{k}\right)}{\int \tau d \nu_{Y, \varepsilon}}+\|\psi\|_{B V} \sum_{k>k_{0}} \sum_{i=0}^{\tau_{k}-1} \frac{\nu_{Y, 0}\left(Y_{k}\right)}{\int \tau d \nu_{Y, 0}} .
\end{aligned}
$$

Now the terms on the second line are bounded by $2 \delta\|\psi\|_{B V}$ and each of the finitely many differences on the right hand side of the first line tend to 0 with $\varepsilon$ by Lemma 6.12 and Proposition 6.10. Since $\delta>0$ was arbitrary, this proves the required convergence of $\nu_{H_{\varepsilon}}$ to $\mu_{t}$ when integrated against functions of bounded variation. To complete the proof of items (1) and (5) of Theorem 1.13 , we extend this convergence to continuous functions by approximation as in the proof of Proposition 6.10. 
6.4.1. Interpretation of $\nu_{H_{\varepsilon}}$. In this section, we prove items (2) and (3) of Theorem 1.13 . We restrict to a sequence of holes $\left(H_{\varepsilon}\right)_{\varepsilon \leqslant \varepsilon_{0}}$ satisfying $(\mathbf{H})$ and such that $\varepsilon_{0}$ is sufficiently small that ${\stackrel{\mathcal{L}}{\mathcal{L}}}_{t \Phi^{H_{\varepsilon_{0}}-\tau p}}{ }^{H_{\varepsilon_{0}}(t)-P_{t, \varepsilon}}$ has a spectral gap by Proposition 6.6

Given a Markov hole $H=H_{\varepsilon}$, set

$$
\psi_{t}^{H}:=t \phi^{H}-p^{H}(t)-P\left(t \Phi^{H}-\tau p^{H}(t)\right) \cdot 1_{Y} .
$$

We first note that

$$
\Psi_{t}^{H}=S_{\tau} \psi_{t}^{H}=t \Phi^{H}-\tau p^{H}(t)-P\left(t \Phi^{H}-\tau p^{H}(t)\right),
$$

so $P\left(\Psi_{t}^{H}\right)=0$.

It follows from [BDM] that the measure $\nu_{Y, \varepsilon}$ constructed in Section 6.3 is a Gibbs measure with Gibbs constant 0 for the potential $t \Phi^{H}-\tau p^{H}(t)-P_{t, \varepsilon}-\log \Lambda_{t, \varepsilon}$. So by Theorem 2.2 .

$$
0=P\left(t \Phi^{H}-\tau p^{H}(t)-P_{t, \varepsilon}-\log \Lambda_{t, \varepsilon}\right)=P\left(t \Phi^{H}-\tau p^{H}(t)\right)-P\left(t \Phi-\tau p^{H}(t)\right)-\log \Lambda_{t, \varepsilon} .
$$

Hence

$$
\log \Lambda_{t, \varepsilon}=P\left(t \Phi^{H}-\tau p^{H}(t)\right)-P\left(t \Phi-\tau p^{H}(t)\right)
$$

and we conclude that $\nu_{Y, \varepsilon}$ is a Gibbs measure for $\Psi_{t}^{H}$. Then by Theorem 2.2, we know that this is an equilibrium state for $\Psi_{t}^{H}$ provided the integral of $\Psi_{t}^{H}$ is finite. This latter fact follows from Lemma 6.11 and since whenever $p^{H}(t)=0$, then $t>\frac{\gamma}{1+\gamma}$ by Proposition 1.6.

Since

$$
0=P\left(\Psi_{t}^{H}\right)=h_{\nu_{Y, \varepsilon}}+\int \Psi_{t}^{H} d \nu_{Y, \varepsilon}=h_{\nu_{Y, \varepsilon}}+\int t \Phi^{H}-\tau p^{H}(t)-P\left(t \Phi^{H}-\tau p^{H_{\varepsilon}}(t)\right) d \nu_{Y, \varepsilon},
$$

Abramov's formula implies

$$
h_{\nu_{H}}+\int \psi_{t}^{H} d \nu_{H}=0
$$

i.e.,

$$
h_{\nu_{H}}+\int t \phi^{H} d \nu_{H}=P\left(t \Phi^{H}-\tau p^{H}(t)\right) \nu_{H}(Y)+p^{H}(t)
$$

In particular, $\nu_{H}$ is an equilibrium state for $\psi_{t}^{H}$, which proves (2) of Theorem 1.13 for Markov holes.

We will next show that the free energy given by (6.17) varies continuously in $\varepsilon$. Using this and the fact that Markov holes are dense in our sequence $\left(H_{\varepsilon}\right)_{\varepsilon \leqslant \varepsilon_{0}}$, we will be able to conclude that in fact (6.17) holds for non-Markov holes as well.

For a given $t \in(0,1)$, note that setting

$$
\varepsilon_{t}:=\inf \left\{\varepsilon \leqslant \varepsilon_{0}: P\left(t \Phi^{H_{\varepsilon}}\right)>0\right\},
$$

we have $P\left(t \Phi^{H_{\varepsilon_{t}}}\right)=0$ whenever $\varepsilon_{t}$ is finite. Moreover, set $\varepsilon_{1}=0$.

We will address two cases, noting that for some values of $t \leqslant t^{H_{\varepsilon_{0}}}$, Case 2 will be empty.

Case 1: $\varepsilon \leqslant \varepsilon_{t}$. In this case $p^{H_{\varepsilon}}(t)=h_{\nu_{H_{\varepsilon}}}+\int t \phi^{H_{\varepsilon}} d \nu_{H_{\varepsilon}}$, and we can show the continuity of this quantity via the Implicit Function Theorem and the fact that in this case $s=p^{H_{\varepsilon}}(t)$ is the unique solution to $P\left(t \Phi^{H_{\varepsilon}}-\tau s\right)=0$.

Case 2: $\varepsilon>\varepsilon_{t}$. In this case $p^{H_{\varepsilon}}(t)=0$ and $h_{\nu_{H_{\varepsilon}}}+\int t \phi^{H_{\varepsilon}} d \nu_{H_{\varepsilon}}=P\left(t \Phi^{H_{\varepsilon}}\right) \nu_{H_{\varepsilon}}(Y)$, so we need to prove continuity of induced pressure and $\nu_{H_{\varepsilon}}(Y)$ in $\varepsilon$. 
Lemma 6.13. Fix $t \in(0,1]$ and $s \in \mathbb{R}$. Let $\left(H_{\varepsilon}\right)_{\varepsilon \in\left[0, \varepsilon_{0}\right]}$ be a collection of holes centered at $z \in I$ with $\varepsilon<\varepsilon^{\prime}$ implying $H_{\varepsilon} \subset H_{\varepsilon^{\prime}}$. If $P(t \Phi-\tau s)<\infty$, then $\varepsilon \mapsto P\left(t \Phi^{H_{\varepsilon}}-\tau s\right)$ is continuous on [0, $\left.\varepsilon_{0}\right]$.

Proof. We will set $s=0$ since the proof for any other value is analogous. Clearly $\varepsilon \mapsto P\left(t \Phi^{H_{\varepsilon}}\right)$ is monotone decreasing. Since we can approximate any hole $H_{\varepsilon}$ by elements of the Markov partition, it is sufficient to consider monotone sequences $\left(H_{\varepsilon_{n}}\right)_{n}$ of Markov holes. We will prove that for $H_{\varepsilon}$, for any $\eta>0$ there exists $n \in \mathbb{N}$ such that $P\left(t \Phi^{H_{\varepsilon} \backslash C_{n}}\right)-P\left(t \Phi^{H_{\varepsilon}}\right)<\eta$ where $C_{n}$ is any $n$-cylinder. This then implies the lemma.

By assumption on $\Phi$, we have $P(t \Phi)<\infty$. Also the spectral radius of $\mathcal{L}_{t \Phi}$ on $B V(Y)$ is $e^{P(t \Phi)}$. On the one hand,

$$
\left|\mathcal{L}_{t \Phi} 1\right|_{L^{1}\left(m_{t}\right)} \leqslant\left\|\mathcal{L}_{t \Phi}\right\|_{B V}\|1\|_{B V}<\infty
$$

while on the other, $\int_{Y} \mathcal{L}_{t \Phi} 1 d m_{t}=\int_{Y} e^{\tau p(t)} d m_{t}$, since $m_{t}$ is $(t \Phi-\tau p(t))$-conformal. Thus $e^{\tau p(t)} \in$ $L^{1}\left(m_{t}\right)$.

We claim that $\stackrel{\circ}{\mathcal{L}}_{t \Phi_{\varepsilon}^{H}}$ is a perturbation of $\mathcal{L}_{t \Phi}$. This follows precisely as in the proof of Lemma 6.7 since

$$
\left|\stackrel{\mathcal{L}}{t \Phi}_{t} \psi-\stackrel{\circ}{\mathcal{L}}_{t \Phi H_{\varepsilon}} \psi\right|_{L^{1}\left(m_{t}\right)} \leqslant|\psi|_{\infty} \int_{\tilde{H}_{\varepsilon} \cup F^{-1} \tilde{H}_{\varepsilon}} e^{\tau p(t)} d m_{t}
$$

and this expression tends to 0 with $\varepsilon$ since $e^{\tau p(t)} \in L^{1}\left(m_{t}\right)$.

Since $P(t \Phi)>0$, it follows from the above that in the perturbative regime $\varepsilon \leqslant \varepsilon_{0}$, we have $P\left(t \Phi^{H_{\varepsilon}}\right)>-t \log 2$. Let $\delta:=P\left(t \Phi^{H_{\varepsilon}}\right)+t \log 2>0$. Since $D F \geqslant 2$, we have $t \Phi \leqslant-t \log 2$ for $t \geqslant 0$, so

$$
t \Phi-P\left(t \Phi^{H_{\varepsilon}}\right) \leqslant-\delta
$$

So for large $n$, subtracting an $n$-cylinder $C_{n}$ from an existing hole adds at most

$$
e^{t S_{n} \Phi(x)} \leqslant e^{-\delta n+n P\left(t \Phi^{H_{\varepsilon}}\right)}
$$

to $Z_{n}\left(t \Phi^{H_{\varepsilon}}\right)$ for $x \in C_{n}$. Briefly denote $Z_{n}=Z_{n}\left(t \Phi^{H_{\varepsilon}}\right)$. Subtracting our $n$-cylinder changes $\frac{1}{n} \log Z_{n}$ to at most $\frac{1}{n} \log \left(Z_{n}+e^{n\left(P\left(t \Phi^{H_{\varepsilon}}\right)-\delta\right)}\right)=\frac{1}{n} \log Z_{n}+\frac{1}{n} \log \left(1+\frac{\left.e^{n\left(P\left(t \Phi^{H_{\varepsilon}}\right)-\delta\right.}\right)}{Z_{n}}\right)$. Since $e^{-n P\left(t \Phi^{H_{\varepsilon}}\right)} Z_{n}$ has subexponential growth, this alters $\frac{1}{n} \log Z_{n}$ by at most $\zeta(n)$ for $\zeta(n)=C e^{-\frac{\delta}{2} n}$ for $C>0$ independent of $n$.

Clearly, $\lim _{k \rightarrow \infty} \frac{1}{m k} \log Z_{m k}(\Psi)=P(\Psi)$ for any Hölder continuous potential $\Psi$. Let $\hat{Z}_{n}=Z_{n}\left(t \Phi^{H_{\varepsilon} \backslash C_{n}}\right)$. Then for $K$ a distortion constant, and $k \in \mathbb{N}, \hat{Z}_{n k} \leqslant K \hat{Z}_{n}^{k}$. Thus

$$
\frac{1}{n k} \log \hat{Z}_{n k} \leqslant \frac{K}{n k}+\frac{1}{n} \log \hat{Z}_{n} \leqslant \frac{K}{n k}+\frac{1}{n} \log Z_{n}+\zeta(n) .
$$

So letting $k \rightarrow \infty$, we see that for $n$ large, $P\left(t \Phi^{H_{\varepsilon}}\right)$ and $P\left(t \Phi^{H_{\varepsilon} \backslash C_{n}}\right)$ differ by $O\left(e^{-\frac{\delta}{2} n}\right)$.

We use the lemma to prove Cases 1 and 2.

Cae 1. Noting that for fixed $t \in \mathbb{R}$ and $\varepsilon \geqslant 0, s \mapsto P\left(t \Phi^{H_{\varepsilon}}-\tau s\right)$ is strictly decreasing, Lemma 6.13 and the Implicit Function Theorem (in its continuous version) imply that $\varepsilon \mapsto p^{H_{\varepsilon}}(t)$ is continuous for $\varepsilon \leqslant \varepsilon_{t}$. 
Case 2. In this case $p^{H_{\varepsilon}}(t)=0$ so the free energy of $\nu_{H_{\varepsilon}}$ is given by $P\left(t \Phi^{H_{\varepsilon}}\right) \nu_{H_{\varepsilon}}(Y)$, according to 6.17). Lemma 6.13 gives continuity of $P\left(t \Phi^{H_{\varepsilon}}\right)$, while continuity of $\nu_{H_{\varepsilon}}(Y)=\int \tau d \nu_{Y, \varepsilon}$ follows from Lemma 6.12 for $\varepsilon \leqslant \varepsilon_{0}$.

6.5. Analyticity of the free energy for $t>t^{H}$. In this section we focus on the pressure (or free energy) of the measures $\nu_{H_{\varepsilon}}=\nu_{H_{\varepsilon}, t}$ constructed previously. Fixing $\varepsilon$ sufficiently small, we drop the subscript $\varepsilon$ and denote $H_{\varepsilon}$ simply by $H$. We will show that the pressure

$$
h_{\nu_{H, t}}+\int t \phi^{H} d \nu_{H, t}
$$

is an analytic function of $t$ for $t>t^{H}$, thus proving item (4) of Theorem 1.13 and completing the proof of that theorem.

For brevity, we will denote $\stackrel{\circ}{\mathcal{L}}_{t \Phi^{H}}$ by $\dot{\mathcal{L}}_{t}$ in this section (recall that $p^{H}(t)=0$ for $t \geqslant t^{H}$ ). Fix $s=\frac{t^{H}+1}{2}$ and for $\psi \in B V(Y)$, write

$$
\stackrel{\mathcal{L}}{t}_{t} \psi(x)=\dot{\mathcal{L}}_{s+(t-s)} \psi(x)=\sum_{y \in F^{-1}(x)} \psi(y) e^{s \Phi^{H}(y)} e^{(t-s) \Phi(y)}=\sum_{n=0}^{\infty} \sum_{y \in F^{-1}(x)} \psi(y) e^{s \Phi^{H}(y)} \frac{(t-s)^{n}}{n !} \Phi(y)^{n},
$$

and the interchange of sums is justified once we show that the series of operators $\sum_{n=0}^{\infty} L_{s, n}$, where $L_{s, n} \psi=\dot{\mathcal{L}}_{s}\left(\psi e^{s \Phi^{H}} \frac{(t-s)^{n}}{n !} \Phi^{n}\right)$, converges in the operator norm on $B V(Y)$. This will follow from our next lemma.

Lemma 6.14. There exists $C_{H}>0$, depending only on $H$ and $f$, but not $t$, such that $\left\|L_{s, n}\right\|_{B V} \leqslant$ $C|t-s|^{n}$. Thus $\dot{\mathcal{L}}_{t}$ is an analytic perturbation of $\dot{\mathcal{L}}_{s}$ for $t \in\left(t^{H}, 1\right)$. Moreover, the perturbation is continuous on the closure $\left[t^{H}, 1\right]$.

Similarly, the operator for the closed system $\mathcal{L}_{t}=\mathcal{L}_{t \Phi}$ is an analytic perturbation of $\mathcal{L}_{s \Phi}$ for $t \in\left(t^{H}, 1\right)$ and continuous on the closure.

Proof. We will show the estimates for $L_{s, n}$ using the punctured potential $\Phi^{H}$. The corresponding estimates for $\Phi$ are nearly identical and are omitted.

Let $K_{j, i}=\left[a_{j, i}, b_{j, i}\right]$ denote the images of intervals of monotonicity for $\stackrel{\circ}{F}$ for which $\tau=j$ and let $\xi_{j}$ denote the inverse branch of $F$ on $K_{j, i}$. Note that by assumption on $H$, there are a finite and uniformly bounded number of $i$ for each $j$, and if $H=\emptyset$, then there is only one $i$ per $j$, thus the inverse $\xi_{j}$ depends only on $j$ and not on $i$. Now for $\psi \in B V(Y)$, we estimate following (6.2) and (6.3),

$$
\begin{aligned}
& \bigvee_{Y} L_{s, n} \psi \\
& \leqslant \sum_{j, i} \bigvee_{K_{j, i}}\left(\psi e^{s \Phi} \frac{(t-s)^{n}}{n !} \Phi^{n}\right) \circ \xi_{j} \\
& +\sum_{j, i}\left|\psi e^{s \Phi} \frac{(t-s)^{n}}{n !} \Phi^{n}\right| \circ \xi_{j}\left(a_{j, i}\right)+\left|\psi e^{s \Phi} \frac{(t-s)^{n}}{n !} \Phi^{n}\right| \circ \xi_{j}\left(b_{j, i}\right) \\
& \leqslant \frac{|t-s|^{n}}{n !}\left[\sum_{j, i} \bigvee_{\xi_{j}\left(K_{j, i}\right)} \psi \cdot \sup _{\xi_{j}\left(K_{j, i}\right)} e^{s \Phi}|\Phi|^{n}+\bigvee_{\xi_{j}\left(K_{j, i}\right)} e^{s \Phi} \Phi^{n} \cdot \sup _{\xi_{j}\left(K_{j, i}\right)}|\psi|+\sum_{j, i} 2 \sup _{\xi_{j}\left(K_{j, i}\right)}|\psi| e^{s \Phi}|\Phi|^{n}\right] \\
& \leqslant \frac{|t-s|^{n}}{n !} \sum_{j, i}\left(\bigvee_{\xi_{j}\left(K_{j, i}\right)} \psi+3 \sup _{\xi_{j}\left(K_{j, i}\right)}|\psi|\right) \sup _{\xi_{j}\left(K_{j, i}\right)} e^{s \Phi}|\Phi|^{n},
\end{aligned}
$$


where in the last line we have used the fact that $e^{s \Phi}|\Phi|^{n}$ is monotonic on each interval $\xi_{j}\left(K_{j, i}\right)$ to replace the variation of the function by its supremum. Since $\sup _{\xi_{j}\left(K_{j, i}\right)} \psi \leqslant C\|\psi\|_{B V}$, we estimate,

$$
\bigvee_{Y} L_{s, n} \psi \leqslant C\|\psi\|_{B V} \frac{|t-s|^{n}}{n !} \sum_{j, i} \sup _{\xi_{j}\left(K_{j, i}\right)} e^{s \Phi}|\Phi|^{n} .
$$

Standard distortion estimates (see (D1) and (D2) of Section 4.3) imply that on each $\xi_{j}\left(K_{j, i}\right)$, $\Phi=-\log D f^{j} \sim \log (j+1)^{\left(1+\frac{1}{\gamma}\right)}$. Thus,

$$
\sup _{\xi_{j}\left(K_{j, i}\right)} e^{s \Phi}|\Phi|^{n} \leqslant C(j+1)^{-s\left(1+\frac{1}{\gamma}\right)}(\log (j+1))^{n} .
$$

Now by (6.1), we have $s>t^{H}>\frac{2 \gamma}{1+\gamma}$ so that $s\left(1+\frac{1}{\gamma}\right)>2$. Thus the sum in 6.18 is bounded by

$$
\sum_{j, i} \sup _{\xi_{j}\left(K_{j, i}\right)} e^{s \Phi}|\Phi|^{n} \leqslant C \sum_{j \geqslant 2} j^{-s\left(1+\frac{1}{\gamma}\right)}(\log j)^{n} \leqslant C^{\prime} \int_{1}^{\infty} x^{-2}(\log x)^{n} d x \leqslant C^{\prime} n !,
$$

where we have integrated by parts $n$ times and used the fact that the number of intervals $K_{j, i}$ is uniformly bounded for each $j$. Putting this estimate together with (6.18) yields,

$$
\sum_{n=0}^{\infty}\left\|L_{s, n}\right\|_{B V} \leqslant C \sum_{n=0}^{\infty}|t-s|^{n}<\infty
$$

since $|t-s|<1$. This proves the claimed bound on $\left\|L_{s, n}\right\|_{B V}$ as well as the analyticity of $\dot{\mathcal{L}}_{t}$.

Using equation (1.4) and the fact that $p^{H}(t)=0$ for $t \geqslant t^{H}$, we have

$$
h_{\nu_{H, t}}+\int t \phi^{H} d \nu_{H, t}=\frac{\log \Lambda_{H, t}+P_{t}}{\int \tau d \nu_{Y, t}},
$$

where $\Lambda_{H, t}$ is the largest eigenvalue of ${\stackrel{\circ}{\mathcal{L}_{t \Phi}{ }^{H}-P_{t}}}$ and $P_{t}=P(t \Phi)$ is the largest eigenvalue of $\mathcal{L}_{t \Phi}$ (both as operators on $B V(Y)$ ). Lemma 6.14 implies that both $\Lambda_{H, t}$ and $P_{t}$ vary analytically in $t$ for $t \in\left(t^{H}, 1\right)$, and are continuous on the closure of this interval. All that remains to consider is the denominator of the fraction on the right hand side of the above expression for the free energy of $\nu_{H, t}$.

Let $\tilde{g}_{t}^{H}$ be the unique probability density in $B V(Y)$ satisfying $\stackrel{\circ}{\mathcal{L}}_{t \Phi^{H}-P_{t}} \tilde{g}_{t}^{H}=\Lambda_{H, t} \tilde{g}_{t}^{H}$. Now since $t \Phi^{H}-P_{t}$ is a contracting potential of bounded variation (see the proof of Lemma 6.1), it follows from [LSV1] that $\stackrel{\circ}{\mathcal{L}}_{t \Phi^{H}-P_{t}}$ admits a conformal measure $\eta_{t}^{H}$ with eigenvalue $\Lambda_{H, t}$, supported on $\stackrel{\circ}{Y}^{\infty}$, such that $\nu_{Y, t}=\tilde{g}_{t}^{H} \eta_{t}^{H}$. (Alternatively one can derive the existence of $\eta_{t}^{H}$ as in Section 4.3 in the proof of Theorem 1.7.)

Since $\stackrel{\llcorner}{\mathcal{L}}_{t \Phi^{H}-P_{t}}$ enjoys a spectral gap by Proposition 6.6. we have the following spectral decomposition for all $\psi \in B V(Y)$,

$$
\stackrel{\circ}{\mathcal{L}}_{t \Phi^{H}-P_{t}} \psi=\Lambda_{H, t} \Pi_{t} \psi+\mathcal{R}_{t} \psi,
$$

where the spectral radius of $\mathcal{R}_{t}$ is strictly smaller than $\Lambda_{H, t}, \Pi_{t} \mathcal{R}_{t}=\mathcal{R}_{t} \Pi_{t}=0$ and $\Pi_{t}^{2}=\Pi_{t}$. Moreover,

$$
\Pi_{t} \psi=\tilde{g}_{t}^{H} \int \psi d \eta_{t}^{H},
$$

and $\Pi_{t}$ is analytic as an operator on $B V(Y)$. It follows that $\int \psi d \eta_{t}^{H}$ also varies analytically for $\psi \in B V(Y)$. 
While $\tau \notin B V(Y)$, we do have $\stackrel{\circ}{\mathcal{L}}_{t \Phi^{H}-P_{t}} \tau \in B V(Y)$. Thus, $\Pi_{t}\left(\stackrel{\circ}{\mathcal{L}}_{t \Phi^{H}-P_{t}} \tau\right)$ is analytic in $t$. It follows from 6.20 and the analyticity of $\tilde{g}_{t}^{H}$, that $\int \dot{\mathcal{L}}_{t \Phi^{H}-P_{t}} \tau d \eta_{t}^{H}$ is analytic in $t$. But by the conformality of $\eta_{t}^{H}$,

$$
\int{\stackrel{\circ}{\mathcal{L}_{t \Phi} H-P_{t}}} \tau d \eta_{t}^{H}=\Lambda_{H, t} \int \tau d \eta_{t}^{H},
$$

and the analyticity of $\Lambda_{H, t}$ allows us to conclude that $\int \tau d \eta_{t}^{H}$ is analytic for $t \in\left(t^{H}, 1\right)$. Combining this with (6.19) proves that the free energy of $\nu_{H, t}$ varies analytically in $t$.

\section{The SWALLOWING CASES}

In this section we describe possible behaviors of escape through a swallowing hole and explore ways in which transitivity on the survivor set can fail. For simplicity, we always assume that $H$ is an interval. Although the following list is not exhaustive (for example, a hole can be a union of intervals and divide the open system into any number of transitive components), further generalizations will be a combination of the situations described below, with some components leaking into others, and the component with the slowest rate of escape dominating the rest.

7.1. Case 1: The hole contains 0. This is the simplest case, when $H=(0, a)$. Then the system is uniformly hyperbolic and the classical analysis for uniformly expanding maps holds: the transfer operator acting on an appropriate space of functions (Hölder continuous in the Markov case, and functions of bounded variation in the non-Markov case) will have spectral gap, the largest eigenvalue will correspond to the escape rate and the corresponding eigenfunction will define a conditionally invariant measure absolutely continuous with respect to the conformal measure $m_{t}$. See [DY] for references.

7.2. Case 2: $H=(a, 1]$ for $a \leqslant 1 / 2$. In this case, only a single branch will remain for the open system and the dynamics are trivial: intervals map progressively to the right until they enter the hole. The escape rate here is clearly $p(t)$ since $\stackrel{\circ}{ }^{n}=\left[0, f^{-n}(a)\right]$ and the conformality of $m_{t}$ implies that $m_{t}\left(J_{k-1}\right)=e^{t S_{k} \phi-k p(t)}$ since $f^{k}\left(J_{k-1}\right)=I$ for each $k \geqslant 1$, so that

$$
\lim _{n \rightarrow \infty} \frac{1}{n} \log m_{t}\left(\stackrel{\circ}{I}^{n}\right)=\lim _{n \rightarrow \infty} \frac{1}{n} \log m_{t}\left(\cup_{k \geqslant n_{a}} J_{k}\right)=-p(t),
$$

where $n_{a}$ is the index such that $f^{-n}(a) \in J_{n_{a}}$.

For $t<1$, Theorem 1.11 gives the existence of physically relevant accim with escape rate $p(t)$. For $t=1$, the only limiting distribution is $\delta_{0}([\mathrm{DF}])$.

7.3. Case 3: Hole to the left of $1 / 2$, not capturing 0 . If $H=(a, b)$ is a collection of adjacent 1-cylinders of $\mathcal{P}_{1}$ strictly between $1 / 2$ and 0 then the system is divided into two components, $L=[0, a]$ and $R=[b, 1]$. Note that $L$ maps to itself and to $H$, but not to $R$, while $R$ maps to itself, to $L$ and to $H$.

The dynamics restricted to $R$ is uniformly hyperbolic and so the classical results hold regarding escape rate and spectral gap for the associated transfer operator. We denote the escape rate out of $R$ by $\log \lambda_{t}^{r}$ and the associated absolutely continuous invariant measure by $\mu_{t}^{r}$. On the other hand, the dynamics on $L$ are completely dominated by the tail as described in Case 2 above.

There are two possibilities for how measures evolve in such a system. If $-\log \lambda_{t}^{r}<p(t)$, then escape is slower from $R$ than from $L$ (note that this cannot happen when $t=1$ ). Since $R$ maps to $L$, the overall escape rate matches that of $R,-\log \lambda_{t}^{r}$, any limiting distribution obtained by pushing 
forward and renormalizing $m_{t}$, i.e. as a limit of $f_{*}^{n} m_{t} /\left|f_{*}^{n} m_{t}\right|$ will be fully supported on $I \backslash H$. In fact, this limiting distribution must be a multiple of $\mu_{t}^{r}$ on $R$.

The second possibility is that $-\log \lambda_{t}^{r}>p(t)$. In this case, the escape rate from $L$ is slower than the escape rate from $R$, but since $L$ does not map to $R$, any limiting distribution obtained by pushing forward and renormalizing $m_{t}$ must be identically 0 on $R$. Any such limit point will have a density on $L$ with escape rate $p(t)$ for $t<1$. For $t=1, \delta_{0}$ will be the only limit point.

Note that both possibilities will occur as $t$ varies in [0,1]. Due to the uniformly hyperbolic behavior on $R$, for Markov holes we have the variational equation for the open system, $-\log \lambda_{t}^{r}=p(t)-p_{r}^{H}(t)$, where $p_{r}^{H}(t)$ is the punctured pressure for the open system on $R$. So the two cases above can be equivalently characterized as $p_{r}^{H}(t)>0$ or $p_{r}^{H}(t)<0$. If $t=0$, we have $p_{r}^{H}(0)$ equal to the topological entropy of the survivor set on $R$, which is positive since $R$ contains a horseshoe; if $t=1$, then $p_{r}^{H}(1)<0$ since $p(1)=0$ and the rate of escape from $R$ with respect to Lebesgue measure is exponential.

7.4. Case 4: $H=(1 / 2, a)$, for some $1 / 2<a<1$. Since $H$ contains a right neighborhood of $1 / 2$, the set $[b, 1]$ is invariant for the open system, where $b=f(a)>0$. As in Case 3, the system splits into two components, $L=[0, b)$ which is single branched and dominated by the tail, and $R=[b, 1]$, which is uniformly hyperbolic. The difference is that now the leakage is from $L$ to $R$ and not from $R$ to $L$.

Considering again the two possibilities, if $-\log \lambda_{t}^{r}<p(t)$ then as above, the overall escape rate matches $-\log \lambda_{t}^{r}$, but now the limiting distribution obtained from $f_{*}^{n} m_{t} /\left|f_{*}^{n} m_{t}\right|$ is simply $\mu_{t}^{r}$ on $R$ and 0 on $L$.

On the other hand, if $-\log \lambda_{t}^{r}>p(t)$, then the overall escape rate will be $p(t)$, and densities will evolve on $L$ as in Case 2 above. For $t<1$, limit points of $f_{*}^{n} m_{t} /\left|f_{*}^{n} m_{t}\right|$ will be fully supported on $I \backslash H$ and the density on $R$ will be a pushforward average of the limiting density on $L$. For $t=1$, $\delta_{0}$ will be the only limit point.

7.5. Case 5: $H=(3 / 4,7 / 8)$. This is anomalous in the sense that if the hole were $H=(3 / 4,1]$, then $H$ would be non-swallowing, so we see that a subset of a non-swallowing hole can be swallowing.

In this case, the system once again divides into two transitive components, $L=[0,3 / 4)$ and $R=[7 / 8,1]$, with $L$ mapping to $R$, but $R$ not mapping to $L$.

The dynamics on $L$ acts as if $H$ were simply $(3 / 4,1]$ and so the theorems of Section 1.4 apply to this system. The dynamics on $R$ are simply those of the doubling map with a single surviving branch, so the classical results for uniformly hyperbolic systems apply to this restricted system. In fact, the escape rate from $R$ is necessarily $p(t)+t \log 2$. The escape rate from $L$ is $-\log \lambda_{t}^{\ell}=p(t)-p_{\ell}^{H}(t)$ by Corollary 1.8, where $p_{\ell}^{H}(t)$ is the punctured pressure for the open system restricted to $L$. So for all $t \in[0,1]$, we have that the escape rate from $L$ is strictly slower than the escape rate from $R$. Thus, for $t<1$ any limit points of $f_{*}^{n} m_{t} /\left|f_{*}^{n} m_{t}\right|$ are fully supported on $I \backslash H$ and are determined by the limiting behavior of the open system on $L$. As usual, for $t=1$ the sole limit point is $\delta_{0}$.

\section{REFERENCES}

[APT] E.G. Altmann, J.S.E. Portela, T. Tél, Leaking chaotic systems, Rev. Mod. Phys. 85 (2013), 869-918.

[BV] W. Bahsoun, S. Vaienti, Metastability of certain intermittent maps, Nonlinearity 25 (2012), 107-124.

[Ba] V. Baladi, Positive transfer operators and decay of correlations, Advanced Series in Nonlinear Dynamics, 16, World Scientific (2000). 
[BJP] O.F. Bandtlow, O. Jenkinson, M. Pollicott, Periodic points, escape rates and escape measures, in Wael Bahsoun, Chris Bose, Gary Froyland, editors, Ergodic Theory, Open Dynamics, and Coherent Structures, pages 41-58, Springer Proceedings in Mathematics and Statistics 70 Springer, New York, 2014.

[B] P. Billingsley, Probability and measure. Wiley Series in Probability and Statistics. John Wiley \& Sons, Inc., Hoboken, NJ, 2012. Anniversary edition.

[BDM] H. Bruin, M.F. Demers, I. Melbourne, Existence and convergence properties of physical measures for certain dynamical systems with holes, Ergodic Theory Dynam. Systems 30 (2010) 687-728.

[BY] L. Bunimovich, A. Yurchenko, Where to place a hole to achieve a maximal escape rate, Israel J. Math. 182 (2011), 229-252.

[BS] J. Buzzi, O. Sarig, Uniqueness of equilibrium measures for countable Markov shifts and multidimensional piecewise expanding maps, Ergodic Theory Dynam. Sys. 23 (2003), 1383-1400.

[C] N.N. Cencova, A natural invariant measure on Smale's horseshoe, Soviet Math. Dokl. 23 (1981), 87-91.

[CM] N. Chernov, R. Markarian, Ergodic properties of Anosov maps with rectangular holes, Bol. Soc. Bras. Mat. 28 (1997), 271-314.

[CMT] N. Chernov, R. Markarian, S. Troubetskoy, Conditionally invariant measures for Anosov maps with small holes, Ergod. Th. and Dynam. Sys. 18 (1998), 1049-1073.

[CV] N. Chernov, H. van dem Bedem, Expanding maps of an interval with holes, Ergod. Th. and Dynam. Sys. 22 (2002), 637-654.

[CMM] P. Collet, S. Martínez, V. Maume-Deschamps, On the existence of conditionally invariant probability measures in dynamical systems, Nonlinearity 13 (2000), 1263-1274.

[CMS] P. Collet, S. Martínez, B. Schmitt, The Yorke-Pianigiani measure and the asymptotic law on the limit Cantor set of expanding systems, Nonlinearity 7 (1994), 1437-1443.

[D1] M.F. Demers, Markov extensions for dynamical systems with holes: An application to expanding maps of the interval, Israel J. Math. 146 (2005), 189-221.

[D2] M.F. Demers, Markov extensions and conditionally invariant measures for certain logistic maps with small holes, Ergod. Th. Dynam. Sys. 25 (2005), 1139-1171.

[D3] M.F. Demers, Dispersing billiards with small holes, in Ergodic theory, open dynamics and coherent structures, Springer Proceedings in Mathematics 70 (2014), 137-170.

[D4] M.F. Demers, Escape rates and physical measures for the infinite horizon Lorentz gas with holes, Dynamical Systems: An International Journal 28 (2013), 393-422.

[DF] M.F. Demers, B. Fernandez, Escape Rates and Singular Limiting Distributions for Intermittent Maps with Holes, Trans. Amer. Math. Soc. 368 (2016), 4907-4932.

[DT] M.F. Demers, M. Todd, Equilibrium states, pressure and escape for multimodal maps with holes, to appear in Israel J. Math.

[DW] M.F. Demers, P. Wright, Behavior of the escape rate function in hyperbolic dynamical systems, Nonlinearity 25 (2012), 2133-2150.

[DWY1] M.F. Demers, P. Wright, L.-S. Young, Escape rates and physically relevant measures for billiards with small holes, Commun. Math. Phys. 294 (2010), 353-388.

[DWY2] M.F. Demers, P. Wright, L.-S. Young, Entropy, Lyapunov exponents and escape rates in open systems, Ergodic Theory Dynam. Sys. 32:4 (2012), 1270-1301.

[DY] M.F. Demers, L.-S. Young, Escape rates and conditionally invariant measures, Nonlinearity 19 (2006), 377397.

[DG] C.P. Dettmann, O. Georgiou, Survival probability for the stadium billiard, Physica D 238 (2009), $2395-2403$.

[DGKK] C.P. Dettman, O. Georgiou, G. Knight, R. Klages, Dependence of chaotic diffusion on the size and position of holes, Chaos 22 023132/1-12 (2012).

[DR] C.P. Dettmann, M.R. Rahman, Survival probability for open spherical billiards, Chaos 24043130 (2014).

[DoW] D. Dolgopyat, P. Wright, The diffusion coefficient for piecewise expanding maps of the interval with metastable states, Stochastics and Dynamics 12 (2012), paper 1150005.

[FP] A. Ferguson, M. Pollicott, Escape rates for Gibbs measures, Ergodic Theory Dynam. Systems, 32 (2012), 961-988.

[FMS] G. Froyland, R. Murray, O. Stancevic, Spectral degeneracy and escape dynamics for intermittent maps with a hole, Nonlinearity 24 (2011), 2435-2463.

[FrP] G. Froyland, K. Padberg-Gehle, Almost-invariant and finite-time coherent sets: directionality, duration, and diffusion, in Wael Bahsoun, Chris Bose, Gary Froyland, editors, Ergodic Theory, Open Dynamics, and Coherent Structures. Proceedings in Mathematics and Statistics 70, pages 171-216, Springer, New York, 2014.

[GHW] C. Gonzalez-Tokman, B. Hunt, P. Wright, Approximating invariant densities for metastable systems, Ergodic Theory Dynam. Systems 34 (2014), 1230-1272.

[HH] H. Hennion, L. Hervé, Limit theorems for Markov chains and stochastic properties of dynamical systems by quasi-compactness, 1766, Lecture Notes in Mathematics, Springer-Verlag, Berlin, 2001. 
[I] G. Iommi, Multifractal analysis for countable Markov shifts, Ergodic Theory Dynam. Systems 25 (2005), 1881-1907.

[IJT] G. Iommi, T. Jordan, M. Todd, Recurrence and transience for suspension flows. Israel J. Math. 209 (2015) 547-592.

[IT1] G. Iommi, M. Todd, Natural equilibrium states for multimodal maps, Comm. Math. Phys. 300 (2010) 65-94.

[IT2] G. Iommi, M. Todd, Dimension theory for multimodal maps, Ann. Henri Poincaré, 12 (2011) 591-620.

[JR] T. Jordan, M. Rams, Multifractal analysis of weak Gibbs measures for non-uniformly expanding $C^{1}$ maps, Ergodic Theory Dynam. Systems 31 (2011) 143-164.

[K] T. Kato, Perturbation Theory for Linear Operators, Classics in Mathematics, Springer: Berlin, Heidelberg, New York, 1980.

[KL1] G. Keller, C. Liverani, Stability of the spectrum for transfer operators, Ann. Scuola Norm. Sup. Pisa Cl. Sci. (4) 28 (1998), 141-152.

[KL2] G. Keller, C. Liverani, Rare events, escape rates and quasistationarity: some exact formulae, J. Stat. Phys. 135 (2009), 519-534

[KM] G. Knight, S. Munday, Escape rate scaling in infinite measure preserving systems, J. Phys. A 49 (2016), paper 85101.

[LM] C. Liverani, V. Maume-Deschamps, Lasota-Yorke maps with holes: conditionally invariant probability measures and invariant probability measures on the survivor set, Annales de l'Institut Henri Poincaré Probability and Statistics, 39 (2003), 385-412.

[LSV1] C. Liverani, B. Saussol, S. Vaienti, Conformal measure and decay of correlation for covering weighted systems, Ergodic Theory Dynam. Systems 18:6 (1998), 1399-1420.

[LSV2] C. Liverani, B. Saussol, S. Vaienti, A probabilistic approach to intermittency, Ergodic Theory Dynam. Systems 19 (1999), 671-685.

[MU] R. Mauldin, M. Urbański, Graph directed Markov systems: geometry and dynamics of limit sets, Cambridge tracts in mathematics 148, Cambridge University Press, Cambridge 2003.

[PY] G. Pianigiani, J. Yorke, Expanding maps on sets which are almost invariant: decay and chaos, Trans. Amer. Math. Soc. 252 (1979), 351-366.

[S1] O. Sarig, Thermodynamic formalism for countable Markov shifts, Ergodic Theory Dynam. Systems 19 (1999), 1565-1593.

[S2] O. Sarig, Thermodynamic formalism for null recurrent potentials, Israel J. Math. 121 (2001) $285-311$.

[S3] O. Sarig, Phase transitions for countable Markov shifts, Comm. Math. Phys. 217 (2001), 555-577.

[S4] O. Sarig, Existence of Gibbs measures for countable Markov shifts, Proc. Amer. Math. Soc. 131 (2003), 17511758 .

[Ya] T. Yarmola, Sub-exponential mixing of random billiards driven by thermostats, Nonlinearity 26 (2013), 18251837.

[Y1] L.S. Young, Some large deviation results for dynamical systems, Trans. Amer. Math. Soc. 318 (1990), $525-543$.

[Y2] L.S. Young, Recurrence times and rates of mixing, Israel J. Math. 110 (1999), 153-188.

[Z] R. Zweimüller, Invariant measures for general(ized) induced transformations, Proc. Amer. Math. Soc. 133 (2005), 2283-2295.

Mark F. Demers, Department of Mathematics, Fairfield University, Fairfield, Ct 06824, USA

E-mail address: mdemers@fairfield.edu

$U R L:$ http://faculty.fairfield.edu/mdemers

Mike Todd, Mathematical Institute, University of St Andrews, North Haugh, St Andrews, KY16 9SS, SCOTLAND

E-mail address: m.todd@st-andrews.ac.uk

$U R L:$ http://www.mcs.st-and.ac.uk/ miket/ 